\title{
Carrier dynamics in semiconductors studied with time-resolved terahertz spectroscopy
}

\author{
Ronald Ulbricht \\ Fundamental Research on Matter (FOM) - Institute for Atomic and Molecular Physics \\ (AMOLF), Science Park 1041098 XG Amsterdam, The Netherlands \\ Euan Hendry \\ Exeter University, School of Physics, Stocker Road, Exeter EX4 4QL, Devon, England
}

Jie Shan

Case Western Reserve University, Department of Physics, 10900 Euclid Avenue, Cleveland, Ohio 44106, USA

Tony F. Heinz

Columbia University, Departments of Physics and Electrical Engineering, New York, New York 10027, USA

Mischa Bonn*

Fundamental Research on Matter (FOM) - Institute for Atomic and Molecular Physics (AMOLF), Science Park 1041098 XG Amsterdam, The Netherlands

(Received 11 February 2010; published 3 June 2011)

\begin{abstract}
Time-resolved, pulsed terahertz spectroscopy has developed into a powerful tool to study charge carrier dynamics in semiconductors and semiconductor structures over the past decades. Covering the energy range from a few to about $100 \mathrm{meV}$, terahertz radiation is sensitive to the response of charge quasiparticles, e.g., free carriers, polarons, and excitons. The distinct spectral signatures of these different quasiparticles in the THz range allow their discrimination and characterization using pulsed $\mathrm{THz}$ radiation. This frequency region is also well suited for the study of phonon resonances and intraband transitions in low-dimensional systems. Moreover, using a pump-probe scheme, it is possible to monitor the nonequilibrium time evolution of carriers and low-energy excitations with sub-ps time resolution. Being an all-optical technique, terahertz time-domain spectroscopy is contact-free and noninvasive and hence suited to probe the conductivity of, particularly, nanostructured materials that are difficult or impossible to access with other methods. The latest developments in the application of terahertz time-domain spectroscopy to bulk and nanostructured semiconductors are reviewed.
\end{abstract}

DOI: 10.1103/RevModPhys.83.543

PACS numbers: $72.20 . J v$

\section{CONTENTS}

I. Introduction

II. Generation and detection of terahertz radiation
A. Generation
1. Generation of $\mathrm{THz}$ radiation by photoconductivity
2. Generation of $\mathrm{THz}$ radiation based on nonlinear optical processes
3. Generation of high-energy $\mathrm{THz}$ pulses
B. Detection
C. A typical THz time-domain spectroscopy setup D. Analysis
1. Obtaining the complex dielectric function
2. Inhomogeneous materials
3. Analysis of photoinduced changes in the dielectric function
545
545
546
546
547
548
549
549
550
550
E. Typical responses observed

544

545
1. Drude response

2. Lorentzian oscillator

F. Comparison to conventional transient photoconductivity

III. Charge carriers in bulk semiconductors

A. Free carriers

1. Determination of scattering times and plasma frequencies in bulk semiconductors

2. Limitations of the Drude model

3. Organic semiconductors

4. Ultrafast dynamics

B. Excitons in bulk materials Intraexcitonic transitions

IV. Nanostructured semiconductors

A. Structures with no quantum confinement

1. Carrier localization

2. Plasmon resonance

3. Localization versus particle plasmons

B. Structures with quantum confinement

*bonn@amolf.nl 
1. Quantum wells

2. Quantum dots

C. Graphitic nanostructures

V. Summary and outlook

\section{INTRODUCTION}

Charge carriers in semiconductors provide the basis for a variety of important technologies, including computers, semiconductor lasers, and light emitting devices. The continuing wish to reduce the physical size of next-generation electronic devices requires increasingly smaller building blocks in electronics. Nanostructures with sizes well below $100 \mathrm{~nm}$, such as semiconductor nanocrystals and nanowires, provide such building blocks. Hence, it is apparent that there is both a technological and fundamental interest in the properties of charge carriers in both bulk and nanostructured materials.

Charge carriers can have very different properties in semiconductors and semiconductor nanostructures, depending on morphology, temperature, and material properties such as the crystal structure, band gap, dielectric function, and electronphonon coupling strength. In a bulk material with high dielectric function, but moderate electron-phonon coupling, charge carriers are efficiently screened from one another, and electrons and holes will be present as free carriers and relatively mobile. For materials with strong electron-phonon coupling, these carrier-lattice interactions will lead to the formation of polarons, carriers that are dressed with local lattice deformations. Polarons have a reduced mobility due to their increased effective mass. For materials with reduced dielectric function, bound electron-hole pairs, excitons, may be formed, which can be thermally dissociated at elevated temperatures.

Regarding the role of morphology of the material on the nature of the charge carriers, one can distinguish several regimes. Consider a material for which in the bulk electrons are present as free carriers. For sufficiently small nanostructures, i.e., of dimension $R$ appreciably smaller than the exciton Bohr radius, strong confinement of carriers occurs. In this limit, the effective gap of the material is increased due to the confinement energy and discrete energy levels are present. For somewhat larger structures with a radius greater than the exciton Bohr radius, but smaller than the electron mean free path $\left(r_{B}<R<l_{f}\right)$, carriers will be free to move, but in a confined volume.

The common characteristic of charge carriers, which may be present in any of the different forms described above, is that they all exhibit a distinct response in the low-frequency range of the electromagnetic spectrum: Exciton binding energies and exciton transitions, for both bulk and nanostructured materials, are typically in the $\mathrm{meV}$ range and are optically active; the response of mobile carriers and polarons is dictated by carrierphonon interactions leading to randomization of the carrier momentum typically occurring on (sub)picosecond time scales, giving rise to dispersion in the dielectric response on meV energy scales. The dielectric response in the same energy range is modified for carriers which undergo a different type of transport (hopping transport or transport in noncrystalline semiconductors). The ability to probe charge carriers in the meV energy or, equivalently, terahertz frequency range, therefore allows their detailed characterization through the distinct spectral signatures in the terahertz range (Grischkowsky et al., 1990; Vanexter and Grischkowsky, 1990; Katzenellenbogen and Grischkowsky, 1992). THz spectroscopy constitutes a contact-free probe of the frequency-dependent conductivity, which is determined by key parameters such as the carrier density and mobility.

$\mathrm{THz}$ time-domain spectroscopy makes use of subpicosecond pulses of freely propagating electromagnetic radiation in the terahertz range. $\mathrm{THz}$ radiation $\left(1 \mathrm{THz}=10^{12} \mathrm{~Hz}\right)$ is characterized by sub-mm wavelengths $(300 \mu \mathrm{m}$ for $1 \mathrm{THz}$ in vacuum), low photon energies $\left(33.3 \mathrm{~cm}^{-1}\right.$ or $4.2 \mathrm{meV}$ at $1 \mathrm{THz}$ ), corresponding to less-than-thermal energies at room temperature (1 THz corresponds to $48 \mathrm{~K}$ ). THz pulses are readily generated by frequency down-conversion of femtosecond optical pulses to the $\mathrm{THz}$ range and detected coherently in the time-domain (see the next section). This brings some pivotal advantages in contrast to traditional far-infrared $\mathrm{cW}$ spectroscopy techniques that were commonly used before [see, e.g., Johnson et al. (1969) and Birch et al. (1974)]. The technique simplifies the experimental setup by not having to use liquid-helium cooled bolometers for detection. It is also insensitive to the blackbody radiation of the environment and can yield very high signal-to-noise ratio measurements (Grischkowsky et al., 1990).

Initially, the available frequency spectrum that could be generated and detected in the time domain was limited to a few THz (Grischkowsky, 1993). Advances in the development of broadband ultrafast lasers and in the fabrication of new nonlinear optical materials have pushed this limit into the midinfrared (Wu and Zhang, 1997; Kaindl et al., 1999; Huber et al., 2000) and recently into the near-infrared, reaching frequencies beyond $100 \mathrm{THz}(\lambda=3 \mu \mathrm{m})$ (Sell, Scheu et al., 2008). This review will be largely limited to $\mathrm{THz}$ time-domain spectroscopy using ultrafast lasers generating $\mathrm{THz}$ pulses - as opposed to continuous wave farinfrared spectroscopy using Fourier transform infrared spectrometers and $\mathrm{THz}$ sources from accelerators such as free-electron lasers. It also excludes works that investigated $\mathrm{THz}$ radiation emitted from materials to study charge carrier and lattice dynamics (Dekorsy et al., 1996; Kadoya and Hirakawa, 2005; Armstrong et al., 2009).

One particular advantage of all-optical generation and detection of $\mathrm{THz}$ pulses is the possibility to combine the approach with a time-synchronized femtosecond excitation pulse. This makes the method well suited for the investigation of electronic charge transport under nonequilibrium conditions [see, e.g., Averitt and Taylor (2002), Beard et al. (2002b), Elsaesser (2004), and Schmuttenmaer (2004)]. This attribute permits $\mathrm{THz}$ spectroscopy to circumvent many of the constraints of conventional transport measurement techniques.

Accordingly, much progress has been made in the past two decades in understanding the physics of elementary electronic excitations, owing to the development of sources and detectors of coherent $\mathrm{THz}$ radiation. Pulsed, time-domain $\mathrm{THz}$ spectroscopy not only allows for the characterization of charge carriers under steady-state conditions, but is also ideally suited for nonequilibrium measurements: using a time-resolved $\mathrm{THz}$ spectroscopy setup, an optical pulse can be used to create charge carriers and the subsequent evolution of charge carriers can be monitored on the femtosecond time 
scale. In this way, one has direct access to the time scales and mechanisms of carrier cooling, trapping, and recombination, as well as the dynamics of formation of quasiparticles such as excitons and polarons.

In this manuscript, we will review the body of work on carrier dynamics in semiconductor and semiconductor nanostructures studied using time-resolved $\mathrm{THz}$ time-domain spectroscopy. The outline of this review is as follows: We start with a description of the technical details and recent advances, and the analysis of $\mathrm{THz}$ signals (Sec. II). This is followed by Secs. III and IV devoted, respectively, to $\mathrm{THz}$ studies of bulk materials and nanostructures. For the bulk materials, we focus on the properties and dynamics of free carriers, polarons, and excitons and the mechanism and time scale of their formation. For the nanostructures, we distinguish structures in which there is no quantum confinement from those where confinement is strong. For the latter, we discuss quantum wells and nanocrystals, the conductivity of nanocrystal assemblies and carbon nanotubes, and graphene. We conclude with a brief outlook in Sec. V.

\section{GENERATION AND DETECTION OF TERAHERTZ RADIATION}

In this section we describe the most common $\mathrm{THz}$ emitters and detectors. We limit the scope primarily to pulsed tabletop sources and detectors that are based on femtosecond lasers and allow electric field-resolved measurements. Therefore, $\mathrm{THz}$ sources such as synchrotrons (Williams, 2004, 2006), freeelectron lasers (Murdin, 2009), quantum-cascade lasers (Faist et al., 2004; Scalari et al., 2009) and gas lasers, and THz detectors such as bolometers and pyroelectric detectors are left out. Details about these sources and detectors can be found in Button (1980) and Woolard et al. (2003). In Secs. II.A and II.B we describe the generation and detection of $\mathrm{THz}$ electromagnetic transients based on either photoconductivity or optical nonlinearity of a medium. We then describe how to combine the generation and detection capabilities for the $\mathrm{THz}$ time-domain spectroscopy (Sec. II.C) and the analysis methods that can be used to extract properties of material of interest in the THz spectral regime (Sec. II.D). The basics of THz time-domain spectroscopy have also been introduced by Grischkowsky (1993), Wynne and Carey (2005), and Lee (2009).

\section{A. Generation}

Photoconductivity and nonlinear optical processes are the two major techniques that have been utilized to generate $\mathrm{THz}$ electromagnetic transients from femtosecond lasers. A description of each of these methods, a comparison of the characteristics of the $\mathrm{THz}$ emission derived from these techniques, and a discussion of approaches for the generation of high-power $\mathrm{THz}$ radiation are included in this section.

\section{Generation of $\mathrm{THz}$ radiation by photoconductivity}

$\mathrm{THz}$ generation based on photoconductivity is a resonant process in which a femtosecond optical pulse is absorbed through interband transitions in a semiconductor to produce charge carriers. These carriers are subsequently accelerated in either an externally applied dc electric field or a built-in electric field in the depletion or accumulation region of the semiconductor. A transient current is thus formed, which in turn emits a $\mathrm{THz}$ electromagnetic transient that can propagate either on a transmission line or in free space (Smith et al., 1988).

For this purpose, the semiconductor can either be incorporated into an antenna or transmission line structure, or it can radiate directly. In the former case, an external electric field is applied across a gap formed by electrodes, which is excited by the optical pulse (Auston, 1983; Auston et al., 1984; Fattinger and Grischkowsky, 1989). The optical pulse is often arranged at normal incidence and the bias field is parallel to the photoconductor surface. The bias field can also be provided by the built-in electric field near the surface of a semiconductor wafer. A depletion or accumulation region is formed in a doped semiconductor as a result of Fermi level pinning. In this case the emitter is excited by an optical pulse at an oblique angle in order to couple the emission into the free space (Hu et al., 1990). The output coupling efficiency is zero for normal incidence. Studies have also shown that magnetic fields can enhance the radiation output coupling efficiency by altering the direction of the current through the Lorentz force (Shan et al., 2001). These studies not only provide conditions to optimize the $\mathrm{THz}$ emission, but also means to investigate ultrafast carrier dynamics in semiconductors in magnetic fields (Shan and Heinz, 2004).

In the far field the emitted $\mathrm{THz}$ electric field is proportional to the first time derivative of the transient current. The current transient is limited by the duration of the optical excitation pulse and the carrier scattering time, as well as the recombination lifetime of the semiconductor and the time that it takes the carriers to drift out of the active emitter area. Therefore, commonly used semiconductors for $\mathrm{THz}$ generation are defect-rich to reduce the fall time of the transient current. Examples include low-temperature grown or ion-implanted GaAs and silicon (McIntosh et al., 1995; Shan and Heinz, 2004; Krotkus and Coutaz, 2005; Mikulics et al., 2006). Following the pioneering work of Auston (1983), Grischkowsky (1993), and their coworkers, researchers optimized ultrafast photoconductive switches in the past two decades to permit generation and field-resolved detection of electromagnetic transients up to $\sim 5 \mathrm{THz}$. Such a bandwidth, while impressive, actually reflects the finite response time of photoconductive materials rather than the ideal bandwidth that could be obtained from current state-of-the-art mode-locked laser pulses. For instance, a 10-fs transform-limited optical pulse (with a bandwidth of $\sim 50 \mathrm{THz}$ ) should in principle permit generation and detection of electromagnetic transients up to $\sim 50 \mathrm{THz}$. In this regime, however, the comparatively slow response of the carriers in available photoconductive media significantly degrades the high-frequency performance. A complete understanding of the frequency response of the emission process can yield insight into a material's carrier dynamics. Upon excitation of a 10-fs optical pulse, the transient photocurrent rises rapidly with a rise time of $10 \mathrm{fs}$ followed by a ballistic acceleration before the onset of the momentum relaxation processes and the carrier recombination processes. The 10-fs rise part of the transient current provides the highest spectral components of the emitted $\mathrm{THz}$ radiation, 
but the spectral bandwidth of the emission is significantly below $\sim 50 \mathrm{THz}$. The spectral bandwidth is determined by the main contributions to the transient current, i.e., the subsequent much slower processes. A careful analysis of the $\mathrm{THz}$ emission has, for example, allowed the investigation of highfield transport on the fs time scale in compound semiconductors such as GaAs and InP (Leitenstorfer et al., 1999, 2000). In contrast, optical rectification, as described in the next section, can potentially generate $\mathrm{THz}$ emission with a bandwidth limited only by the duration of the optical excitation pulse. Under the assumption of perfect phase matching and a second-order nonlinearity of the emitter independent of frequencies in the region of interest, the emitted $\mathrm{THz}$ electric field in the far field is proportional to the second time derivative of the nonlinear polarization which follows the intensity envelope of the excitation pulse.

With respect to the strength of the $\mathrm{THz}$ emission, a linear dependence of the $\mathrm{THz}$ electric field on the dc bias (Darrow et al., 1991; Reimann, 2007) has been observed. At low excitation fluence, the $\mathrm{THz}$ field also varies linearly with fluence; however, high excitation fluence often leads to saturation of the $\mathrm{THz}$ emission. There are two main reasons for saturation: (i) the resultant high charge densities effectively screen the bias electric field; and (ii) the electric-field of the emitted radiation acts back and further decreases the net bias field (Benicewicz et al., 1994; Kim and Citrin, 2006). Photoconductive antennas and coplanar transmission lines with a small gap (tens of microns) are often used with a femtosecond oscillator source that delivers optical pulses of energy on the order of $10^{-9} \mathrm{~J} /$ pulse. A bias electric field of $\sim 10^{6} \mathrm{~V} / \mathrm{m}$ can be applied and a typical $\mathrm{THz}$ pulse energy of $\sim 10^{-13} \mathrm{~J}$ (and of peak power of $10^{-5} \mathrm{~W}$ ) can be achieved. With an amplified femtosecond laser source that delivers pulses of energy on the order of $10^{-3} \mathrm{~J} /$ pulse, to avoid saturation, large-aperture structures $(\sim \mathrm{mm}$ gap size $)$ or bare semiconductor wafers are often used. $\mathrm{THz}$ emission with a peak electric field up to $150 \mathrm{kV} / \mathrm{cm}$, corresponding to an energy of $10^{-7} \mathrm{~J} /$ pulse and a peak power of $10^{5} \mathrm{~W}$, has been reported (You et al., 1993). Details can be found in Sakai (2005) and Cheville (2008).

\section{Generation of $\mathrm{THz}$ radiation based on nonlinear optical processes}

An alternative method to generate $\mathrm{THz}$ radiation is to rely on nonresonant nonlinear optical processes such as optical rectification. Optical rectification is a second-order nonlinear process in which a dc or low-frequency polarization is developed when an intense laser beam propagates through a non-centro-symmetric crystal. It can be viewed as differencefrequency generation between the frequency components within the band of an optical excitation pulse. In contrast to photoconductivity, it is a nonresonant process and can therefore withstand higher excitation fluences and, importantly, generate $\mathrm{THz}$ emission with a bandwidth limited only by that of the optical excitation pulse.

In choosing appropriate nonlinear crystals for $\mathrm{THz}$ generation, several factors need to be considered: [for more details, see Reimann (2007)]

(i) The achievable $\mathrm{THz}$ bandwidth is always fundamentally limited by the bandwidth of the laser excitation pulse. As an example, a Fourier-transform limited $100 \mathrm{fs}$ pulse at $800 \mathrm{~nm}$ wavelength is characterized by a width of $10 \mathrm{~nm}$ which translates to a maximum $\mathrm{THz}$ bandwidth of about $5 \mathrm{THz}$.

(ii) The material should possess a large nonlinear susceptibility combined with a high damage threshold.

(iii) The material should be transparent throughout the desired frequency range, in both the terahertz and optical regimes. Unfortunately, most of the commonly used inorganic crystals exhibit phonon modes between 5 and $10 \mathrm{THz}$.

(iv) For efficient nonlinear processes, the phase-matching condition has to be fulfilled. For optical rectification, this requires that the group velocity of the excitation pulse matches the phase velocity of all frequency components of the emitted $\mathrm{THz}$ pulse (Nahata, Weling, and Heinz, 1996). For instance, for an optical excitation pulse centered at $800 \mathrm{~nm}(1.55 \mathrm{eV}), \mathrm{ZnTe}$ and $\mathrm{GaP}$ with an optical gap around $2.3 \mathrm{eV}$ have a coherence length exceeding a $\mathrm{mm}$ for $\mathrm{THz}$ frequencies up to 2.2 THz (Nahata, Weling, and Heinz, 1996).

Based on the above requirements, standard choices for nonlinear crystals for optical rectification using a Ti:sapphire laser are $\mathrm{ZnTe}$ for the $0-3 \mathrm{THz}$ range, $\mathrm{GaP}$ for $2-7 \mathrm{THz}$, and GaSe for 8-40 THz (Wu and Zhang, 1997; Kaindl et al., 1999; Huber et al., 2000). There have also been considerable efforts in the development of organic materials for optical rectification. Examples include 4-dimethylamino- $N$-methyl4-stilbazolium-tosylate (DAST) (Katayama et al., 2010), $2 \mathrm{~N}$-a-(methylbenzylamino)-5-nitropyridine (NBANP) crystals, and dye-doped polymers (Zheng et al., 2007). Organic materials often possess larger nonlinearities than inorganic materials, but may exhibit relatively low damage threshold or a lack of photostability.

Plasmas have recently been recognized as another attractive nonlinear medium for $\mathrm{THz}$ generation. The lack of absorbing phonon modes in plasma permits the generation of broadband radiation without spectral gaps. Spectral components up to $75 \mathrm{THz}$ have been observed and center frequencies up to $30 \mathrm{THz}$ have been predicted for an excitation pulse of $50 \mathrm{fs}$ duration (Kim et al., 2008). Plasmas have also been shown to generate intense $\mathrm{THz}$ radiation, with energies exceeding $>5 \mu \mathrm{J}$ per pulse (Kim et al., 2008). In these experiments a focused fundamental pulse at $800 \mathrm{~nm}$ is mixed with its second harmonic at $400 \mathrm{~nm}$ in a gas (Cook and Hochstrasser, 2000). The fundamental beam ionizes the gas in its focus, which then acts as the nonlinear medium. Several groups have worked on understanding and optimizing this process. Researchers examined the dependence of the $\mathrm{THz}$ emission on the parameters, such as the phase and polarization of the optical excitation (Xie et al., 2006; Houard et al., 2008), the optical pulse duration (Bartel et al., 2005), the external dc bias (Thomson et al., 2007), and the type of gas (Chen, Yamaguchi et al., 2007; Lu et al., 2008). However, the underlying microscopic mechanism of the process is still a subject of debate (Thomson et al., 2007; Karpowicz et al., 2009).

\section{Generation of high-energy $\mathrm{THz}$ pulses}

In most studies $\mathrm{THz}$ radiation is used to probe the material response without perturbation, as expected for typical 
conditions of a peak electric-field amplitude up to a few $\mathrm{kV} / \mathrm{cm}$ and a pulse energy of $\sim 10^{-10} \mathrm{~J}$. THz pulses of high energy are thus not required. It is, however, of great interest to be able to study the response of charge carriers and low-energy excitations in the nonlinear regime, thus requiring $\mathrm{THz}$ pulses of high energy as a pump. The advent of amplified Ti:sapphire systems supplying near-infrared pulses in the $\mathrm{mJ}$ regime and novel $\mathrm{THz}$ generation schemes have yielded $\mathrm{THz}$ radiation with energies in the range of $\mu \mathrm{J} /$ pulse. Such a pulse energy is comparable to that of subpicosecond $\mathrm{THz}$ pulses generated by free-electron lasers (Carr et al., 2002).

An obvious approach to scale up the $\mathrm{THz}$ emission based on nonlinearity is to use higher fluences of the optical excitation pulse. This approach is often limited by two-photon absorption in the nonlinear material. The photoinduced charge carriers screen a substantial part of the generated $\mathrm{THz}$ radiation, thereby limiting the conversion efficiency. To circumvent this problem, one can either enlarge the pump beam to lower the excitation pulse fluence while maintaining the total energy or use wide band gap materials. For instance, it has been shown that $\mathrm{THz}$ pulses of $1.5 \mu \mathrm{J}$ energy at a center frequency of $0.6 \mathrm{THz}$ can be generated with an optical pulse of $48 \mathrm{~mJ} /$ pulse in a ZnTe crystal of $75 \mathrm{~mm}$ size (Blanchard et al., 2007). THz pulses of energy up to $30 \mu \mathrm{J} /$ pulse at a center frequency of $0.6 \mathrm{THz}$ were also generated using a $28 \mathrm{~mJ}$ optical pulse (Stepanov et al., 2008) from magnesium-doped lithium niobate $\left(\mathrm{Mg}: \mathrm{LiNbO}_{3}\right)$. This material has a high damage threshold and a large nonlinear susceptibility (Hebling, Yeh, Hoffman, Bartal, and Nelson, 2008; Hebling, Yeh, Hoffman, and Nelson, 2008), but significant phonon absorption above $1 \mathrm{THz}$ (Bartal et al., 2007).

The criterion to drive nonlinear processes with $\mathrm{THz}$ radiation is not, however, the pulse energy, but rather the pump intensity or its electric-field strength. Long-wavelength beams like the ones created in the aforementioned studies suffer from reduced focusability. Thus, it is difficult to reach peak amplitudes beyond $100 \mathrm{kV} / \mathrm{cm}$ (Loffler et al., 2005). The situation becomes easier for higher-frequency beams. Pulses with a spectrum from 1 to $7 \mathrm{THz}$ created in laserinduced plasma have been shown to reach peak amplitudes of $400 \mathrm{kV} / \mathrm{cm}$, despite a pulse energy of just $30 \mathrm{~nJ}$ (Bartel et al., 2005).

With photoconductive switches, one can scale up the $\mathrm{THz}$ emission by increasing both the bias field and the optical excitation fluence. The screening effects by the photoexcited carriers can be significantly reduced by using a large-aperture structure with a gap size of up to a few $\mathrm{mm}$. The excitation power and the bias voltage are scaled up to maintain the magnitude of the excitation fluence and bias field.

On the other hand, for applications such as imaging and steady-state spectroscopy, high average $\mathrm{THz}$ power is desirable. While the ultrafast laser-based techniques can generate $\mathrm{THz}$ radiation of an average power up to $10^{-4} \mathrm{~W} ; 20 \mathrm{~W}$ has been reported for free-electron lasers (Carr et al., 2002). Recent developments in quantum-cascade lasers (QCL) and high-frequency electronics have demonstrated the possibility of providing $\mathrm{cW} \mathrm{THz}$ radiation up to $\sim 100 \mathrm{~mW}$ (Williams, 2006, 2007). The development of quantum-cascade lasers has seen rapid progress since the first prototype was fabricated in 1994 (Faist et al., 1994). They can now offer reliable cw or pulsed mode operation in the midinfrared at room temperature (Williams, 2007; Liu et al., 2010). Scaling down of the emission to $\mathrm{THz}$ frequencies is however technically challenging (Scalari et al., 2009). The first QCL that emitted light at $\mathrm{THz}$ frequencies was only demonstrated in 2002 (Kohler et al., 2002). THz QCLs still require cryogenic working temperatures and commercially available models are scarce. We are currently not aware of any applications in spectroscopy. Some groups have, however, used $\mathrm{THz}$ spectroscopy to study lasing and charge carrier dynamics in QCLs. We briefly treat some of these studies in Sec. IV.B.1.

\section{B. Detection}

Like the generation process, detection of $\mathrm{THz}$ electromagnetic transients based on a femtosecond laser can be achieved by either a photoconductive or nonlinear optical method (Cai et al., 1998). The working principle of a photoconductive antenna detector is similar to the emitter case. Here an optical probe pulse and a $\mathrm{THz}$ pulse simultaneously interact with the switch, with the former producing charge carriers and the latter driving them to form a current. The electric field (including both its amplitude and sign) associated with the $\mathrm{THz}$ radiation at the instant of overlap with the optical probe pulse is therefore determined from the photoinduced current. To obtain the entire waveform of the $\mathrm{THz}$ electromagnetic transient, one simply needs to apply a sampling technique, by varying the time delay between the $\mathrm{THz}$ and the optical probe pulses (Cai et al., 1998; Shen et al., 2004).

Another method of detection of THz electromagnetic transients is to employ the electro-optic (EO) effect. The linear EO effect (also known as the Pockel's effect) produces a birefringence in materials with inversion symmetry upon application of a bias electric field (Wu and Zhang, 1995; Nahata, Auston, and Heinz, 1996). In this case the electric field associated with the $\mathrm{THz}$ radiation acts as the bias field. The induced birefringence then causes a rotation of the polarization of the probe optical beam, which is measured from the optical power transmitted through the EO crystal surrounded by two crossed polarizers. To obtain the entire waveform, a sampling scheme can be used just as for photoconductive detection. Because the same mechanism is involved in both generation and detection, identical materials can be used for emitters and detectors. A more comprehensive review of both methods of $\mathrm{THz}$ detection, with a detailed description of the underlying physics, can be found in Reimann (2007).

Below we note a few key properties of the EO detection method of the $\mathrm{THz}$ radiation.

(i) Multichannel detectors can be easily incorporated into the detection scheme for either spatial imaging of the $\mathrm{THz}$ radiation or detection of the $\mathrm{THz}$ electric-field waveform in a single laser shot. The latter can be achieved by translating the time dependence into spectral dependence using a chirped optical probe beam (Jiang and Zhang, 1998), into spatial dependence in a noncollinear geometry of the $\mathrm{THz}$ and optical beams (Shan et al., 2000), or through other methods (Yellampalle et al., 2005; Kim et al., 2007; Kawada et al., 2008). 
(ii) The transmitted optical probe power through an EO crystal between two crossed polarizers varies with the phase shift caused by the birefringence in the system as $\sim \sin ^{2}\left[\left(\varphi+\varphi_{0}\right) / 2\right]$. Here $\varphi$ is the field-induced phase shift, which is linearly proportional to the $\mathrm{THz}$ electric field, and $\varphi_{0}$ is a field-independent constant phase shift, the origin of which is discussed below. Such a relationship can be linearized around the constant phase $\varphi_{0}$ if $\varphi_{0} \gg \varphi$. A phase shift of $\varphi_{0}=\pi / 4$ is introduced to obtain the greatest absolute modulation in the probe beam intensity for a given $\mathrm{THz}$ field strength (Wu and Zhang, 1995; Nahata, Auston, and Heinz, 1996), and a near-zero phase shift is introduced for measurements that benefit from a higher modulation depth of the probe beam (Jiang et al., 1999; Shan et al., 2000). In a cubic material such as ZnTe and GaP (with no birefringence ideally) the former can be achieved by making use of a quarter wave plate and the latter by relying on the residual birefringence in the crystals (for instance, due to stress). However, in the shot noise limit both approaches yield a similar signalto-noise ratio (Jiang et al., 1999).

(iii) One major advantage of the EO detection method is its time resolution, which is, in principle, limited only by the duration of the optical probe pulse. To achieve such ideal time resolution, crystals with good phasematching properties and thin crystals are often employed. Han and Zhang (2001), Huber et al. (2000), and coworkers demonstrated a detection bandwidth $>30 \mathrm{THz}$ using free-space EO sampling in thin inorganic semiconductor crystals such as ZnTe, GaP, and GaSe. Cao et al. (2002) did the same with poled polymers. By combining a thin GaSe emitter $(90 \mu \mathrm{m})$ and a thin ZnTe detector $(10 \mu \mathrm{m})$, Huber et al. (2000) were able to obtain $\mathrm{THz}$ pulses shorter than 50 fs (bandwidth $\sim 40 \mathrm{THz}$ ) based on a 10-fs laser. The highest spectral components that have been demonstrated using electro-optical sampling to date exceed 135 THz (Sell, Leitenstorfer, and Huber, 2008).

\section{A typical THz time-domain spectroscopy setup}

The basic techniques for $\mathrm{THz}$ generation and detection have been outlined above from the point of view of the fundamental processes of photoconductivity and optical nonlinearity. In this section we introduce a typical experimental setup for performing $\mathrm{THz}$ time-domain spectroscopy (TDS). A schematic representation of a typical THz TD spectrometer is shown in Fig. 1. It consists of a mode-locked laser, a $\mathrm{THz}$ emitter and detector, and elements to couple and propagate $\mathrm{THz}$ radiation from the emitter to the detector. The modelocked laser provides a train of femtosecond optical pulses that are divided into two arms. One laser pulse is used to excite the emitter (either a photoconductor or a second-order nonlinear crystal). The second pulse, introduced at a defined time by an optical delay line, is used to detect the generated $\mathrm{THz}$ radiation in the detector (either a photoconductive switch or an electro-optic crystal).

In the experimental setup, the mode-locked laser is often a Ti:sapphire laser that typically delivers pulses of less than

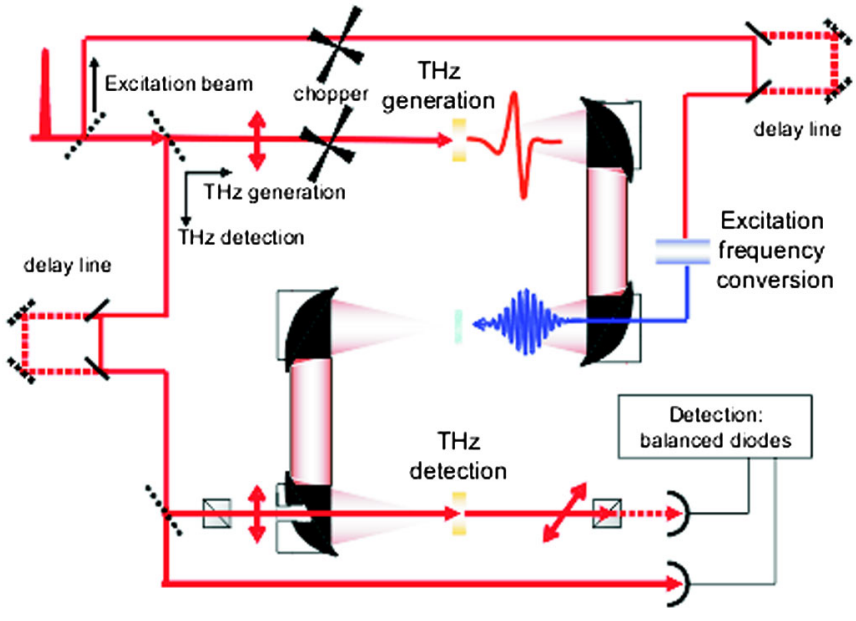

FIG. 1 (color online). Schematic representation of an optical pump-THz probe experimental setup. A train of ultrashort laser pulses enters the setup from the upper left corner and is split into three: the excitation beam, and two beams for the generation and detection of THz pulses. A mechanical chopper is placed in either the excitation or generation beam, depending on the type of experiment. In the balanced detection scheme, the $\mathrm{THz}$ fieldinduced rotation of the polarization of the detection pulse is measured as indicated. Two optical delay lines are used to control the delay times between the three pulses.

$100 \mathrm{fs}$ duration at a wavelength near $800 \mathrm{~nm}$. An important characteristic of the laser system is its stability in both power and pointing direction because the technique relies on separate sample and reference measurements to determine the sample properties. For certain applications, such as ultrabroadband $\mathrm{THz}$ spectroscopy, the stability of the duration of the optical pulse also plays a key role in the system performance. Recently, mode-locked fiber lasers based Er/ Yt-doped glass (Fermann and Hartl, 2009) have emerged as attractive sources for compact THz-TDS setups (Sell, Scheu et al., 2008). Many of the emitters and detectors discussed in previous sections are optimized for operation with Ti:sapphire lasers at a wavelength of $800 \mathrm{~nm}$, rather than at the $1550 \mathrm{~nm}$ wavelength of the mode-locked fiber lasers. However, progress has been reported in the development of emitters and detectors suitable for these new fiber laser sources (Chang et al., 2006; Pacebutas et al., 2010). These compact systems are expected to play an increasingly important role in the future of $\mathrm{THz}$ spectroscopy.

A critical element in the THz-TDS system is the efficient coupling between the emitter and detector, which is of particular importance for photoconductive switch-based $\mathrm{THz}$ systems. Lenses of silicon or other high index materials are often attached to the emitter and detector for efficient coupling. In addition, parabolic mirrors are used to guide the $\mathrm{THz}$ radiation in free space from the emitter to the detector. Two configurations are commonly used in $\mathrm{THz}$ spectrometers: a confocal system consisting of a pair of parabolic mirrors that has a frequency-dependent beam waist at the midpoint and the emitter and detector in the focal planes; and a system consisting of two pairs of parabolic mirrors arranged to have the emitter, detector, and the midpoint in the confocal planes. The latter is often used in $\mathrm{THz}$ imaging and optical pump-THz probe systems. To reduce absorption of the $\mathrm{THz}$ 
radiation by water vapor, the spectrometer is often purged with dry air or nitrogen gas. More details about the THz-TDS systems can be found in Beard et al. (2002b), Cheville (2008), and Lee (2009).

The THz-TDS system can be easily converted into an optical pump-THz probe setup by introducing an optical pump pulse. The pump pulse is derived from the same mode-locked laser, as shown in Fig. 1. The optical pump and the $\mathrm{THz}$ probe spatially overlap at the sample. The time delay between the two pulses can be varied by adjusting an optical delay line.

\section{Analysis}

The THz-TDS system described above is capable of generating and detecting the electromagnetic transients on the femtosecond time scale. To use these capabilities to characterize material properties in the $\mathrm{THz}$ spectral regime, a measurement of the $\mathrm{THz}$ electric-field waveform is first obtained without the sample in place $\left[E_{\text {ref }}(t)\right]$ and then with the sample in place $[E(t)]$, in either a reflection or transmission geometry (Grischkowsky, 1993). Below we describe how to extract the material properties as reflected in the complex dielectric function (or equivalently the complex refractive index or conductivity) from the measurements.

\section{Obtaining the complex dielectric function}

The linear response of material can be described by its complex dielectric function $\epsilon(\nu)$ as a function of the frequency $\nu$. The complex dielectric function is directly related to the complex refractive index $n(\nu)$ by $\epsilon(\nu)=n^{2}(\nu)$, and to the optical conductivity $\sigma(\nu)$ by $\sigma(\nu)=-2 \pi i \nu \epsilon_{0}[\epsilon(\nu)-$ $1]$, where $\epsilon_{0}$ is the vacuum permittivity. The goal of a THzTDS experiment is usually to extract this material response. In this section, we discuss the general procedure for doing so.

The material response can be extracted through the Fourier transforms of the measured electric-field waveforms for the sample $E(\nu)$ and the reference $E_{\text {ref }}(\nu)$. The transmission $t(\nu)$ or reflection $r(\nu)$ of the electric field $\left[=E(\nu) / E_{\text {ref }}(\nu)\right]$ can be related to the parameters of the sample. In the general case of a multilayered planar system of $N$ layers, a transfer matrix analysis can be applied (Furman, 1996). For simplicity, we assume the permeability of the materials to be 1 . The characteristic matrix for normal incidence at $m$ th layer of thickness $d_{m}$ and dielectric function $\epsilon_{m}$ is given by

$$
z_{m}\left(\epsilon_{m}, \nu\right)=\left[\begin{array}{cc}
\cos \left(\beta_{m}\right) & -\frac{i}{\sqrt{\epsilon_{m}}} \sin \left(\beta_{m}\right) \\
-\frac{i}{\sqrt{\epsilon_{m}}} \sin \left(\beta_{m}\right) & \cos \left(\beta_{m}\right)
\end{array}\right]
$$

where $\beta_{m}=2 \pi \nu d_{m} \sqrt{\epsilon_{m}} / c$ is the phase delay associated with propagation inside the $m$ th layer and $c$ is the speed of light in vacuum. The characteristic matrix of the entire multilayer system is given by the product of individual layer matrices $z=z_{N} z_{N-1} \cdots z_{1}$. The transmission and reflection coefficient of the system are determined by the matrix elements $(i, j=1,2)$ of the characteristic matrix of the entire system $z$ :

$$
\begin{aligned}
& r\left(\epsilon_{1}, \epsilon_{2}, \ldots, \epsilon_{N}, d_{1}, d_{2}, \ldots, d_{N}, \nu\right)=\frac{\left(z_{11}+z_{12}\right)-\left(z_{21}+z_{22}\right)}{\left(z_{11}+z_{12}\right)+\left(z_{21}+z_{22}\right)}, \\
& t\left(\epsilon_{1}, \epsilon_{2}, \ldots, \epsilon_{N}, d_{1}, d_{2}, \ldots, d_{N}, \nu\right)=\frac{2}{\left(z_{11}+z_{12}\right)+\left(z_{21}+z_{22}\right)} .
\end{aligned}
$$

In case of a slab of homogeneous material of thickness $d$ and dielectric function $\epsilon_{2}(\nu)$ with media 1 and 3 on its two sides, a common experimental geometry, one retrieves the following more familiar expression for the transmission coefficient:

$$
t\left(\epsilon_{2}, \nu\right)=\frac{E(\nu)}{E_{\mathrm{inc}}(\nu)}=\frac{t_{12} t_{23} e^{i \beta_{2}}}{1+r_{12} r_{23} e^{i 2 \beta_{2}}} .
$$

Here $t_{i j}=2 \sqrt{\epsilon_{i}} /\left(\sqrt{\epsilon_{i}}+\sqrt{\epsilon_{j}}\right) \quad$ and $\quad r_{i j}=\left(\sqrt{\epsilon_{i}}-\right.$ $\left.\sqrt{\epsilon_{j}}\right) /\left(\sqrt{\epsilon_{i}}+\sqrt{\epsilon_{j}}\right)$ are the Fresnel transmission and reflection coefficients for normal incidence to an interface from medium $i$ to $j$ and $E_{\text {inc }}(\nu)$ is the incident field. The task is then to solve Eq. (2) using experimentally determined $E(\nu)$ and $E_{\text {inc }}(\nu)$ and knowledge of the sample thickness to obtain $\epsilon_{2}(\nu)$. Generally, this will involve numerical calculations: Eq. (2) can be solved either using an iterative approach or piecewise in frequency to minimize phase and amplitude errors (Duvillaret et al., 1996).

In the limit of a thin-film sample of thickness $d \ll \lambda_{\mathrm{THz}}$ (where $\lambda_{\mathrm{THz}}$ is the wavelength of $\mathrm{THz}$ radiation), an analytical solution for the film dielectric function $\epsilon(\nu)$ is possible. Here we take the $\mathrm{THz}$ transmission through the film on a substrate as $E(\nu)$ and the $\mathrm{THz}$ radiation transmitted through the bare substrate as $E_{\text {ref }}(\nu)$. One can carry out a Taylor expansion of the exponential terms in Eq. (2) to obtain

$$
\frac{E(\nu)}{E_{\mathrm{ref}}(\nu)}=\frac{1+i \beta}{1+(i 2 \pi \nu d / c)\left[1+(\sqrt{\epsilon}-1)\left(\sqrt{\epsilon_{\mathrm{sub}}}-\sqrt{\epsilon}\right) /\left(1+\sqrt{\epsilon_{\mathrm{sub}}}\right)\right.},
$$

where $\epsilon_{\text {sub }}$ is the substrate dielectric function. Equation (3) can be readily solved analytically to obtain $\epsilon(\nu)$.

We note that in deriving the characteristic matrix described in Eq. (1), we assume that the THz beam can be represented as a superposition of plane waves of frequency $\nu$ propagating along a fixed direction (normal to the sample in this case) and the beam shape is not altered by the sample. These assumptions are usually valid. In case of significant diffraction, the spatial profile of the $\mathrm{THz}$ pulse needs to be measured and a spatial Fourier transform needs to be used in addition to the temporal Fourier transform above.

The measurement technique and the analysis method described here are very general. They can be applied to material systems ranging from dielectrics to semiconductors to metals, in gas, liquid, and solid forms. They can be applied to a homogeneous sample or a composite. In the next Sec. II.D.2 we describe how to extract the dielectric properties of constituents of a composite based on the effective medium theory 
(EMT). The THz TDS technique and the analysis method described above, however, have restrictions. We list some of the major ones below.

(i) The dynamic range (ratio of the peak electric field to the minimum detectable field) of THz-TDS is typically large. For photoconductive antenna-based systems, dynamic ranges over 10000 have been achieved. However, the precision of the measurements is usually limited by the laser noise, which is typically $0.1 \%-1 \%$. This in turn places a minimum limit on the thickness of a sample for which the complex dielectric function can be reliably inferred (typically $\left.|\sqrt{\epsilon}|(2 \pi \nu / c) d>10^{-2}\right)$. To measure samples with a weak effect on the $\mathrm{THz}$ radiation, modulation techniques are often required. For instance, the sample can be moved in and out of the beam, with the difference between the signal with and without the sample, $E(t)-E_{\text {ref }}(t)$, being detected by a phase-sensitive detector. More details can be found in Jiang et al. (2000), Brucherseifer et al. (2002), and Mickan et al. (2002).

(ii) Because the analysis of $\mathrm{THz}$ measurements involves a Fourier transform of the electric-field waveforms, the bandwidth of the measurement is numerically limited by the Nyquist frequency, $(2 \Delta t)^{-1}$, where $\Delta t$ is the time step of the delay line. Typically, $\Delta t$ is chosen so that the Nyquist frequency is 2 or 3 times the physical bandwidth of the system.

(iii) The spectral resolution $\Delta \nu$ of the THz TDS is determined by the duration of the measured waveforms. For a fixed step size $\Delta t$ and $N$ data points acquired, the resolution is given by $\Delta \nu=(2 N \Delta t)^{-1}$. For instance, for a 100-ps measurement scan, this corresponds to a spectral resolution of $\sim 5 \mathrm{GHz}$. Such a resolution is usually not an issue for solids or liquids that have broad resonances, but the linewidths of gases are often limited by the instrumental spectral resolution.

\section{Inhomogeneous materials}

In Sec. II.D.1 we considered homogeneous media. The analysis is also applicable to inhomogenous materials with inhomogeneities on a length scale much smaller than the wavelength of the $\mathrm{THz}$ radiation. These systems include nanocomposites and nanoporous materials. An effective dielectric function $\epsilon(\nu)$ can be extracted from the THz measurements. In order to obtain the properties of the constituents, an effective medium theory is usually applied.

There are many EMTs in Choy (1999). Maxwell-Garnett (MG) (Garnett, 1904) and Bruggemann (1935) are the two most widely used ones. For particles made of a material of dielectric function $\epsilon_{p}$ imbedded in a medium of dielectric function $\epsilon_{m}$, the effective dielectric function of the composite $\epsilon$ is the solution of the following self-consistent equations:

$$
\begin{aligned}
& \text { MG: } \frac{\epsilon-\epsilon_{m}}{\epsilon+\kappa \epsilon_{m}}=f \frac{\epsilon_{p}-\epsilon_{m}}{\epsilon_{p}+\kappa \epsilon_{m}}, \\
& \text { Bruggemann: } \quad f \frac{\epsilon_{p}-\epsilon}{\epsilon_{p}+\kappa \epsilon}=(f-1) \frac{\epsilon_{m}-\epsilon}{\epsilon_{m}+\kappa \epsilon} .
\end{aligned}
$$

Here $f$ is the filling factor in volume and $\kappa$ is the geometric factor related to depolarization ( $\kappa=2$ for spheres) (Garnett, 1904). In contrast to the Bruggemann approximation, in Maxwell-Garnett EMT the role of the particles and their surroundings is not symmetric and is applicable only for dilute composites. The general consensus is that Bruggemann formula is more appropriate for composites consisting of two or more components at high filling factors. However, it is well known that the Bruggemann effective medium theory does not reproduce some important material properties, such as plasmon resonances in percolated conductors (Riikonen et al., 2005).

\section{Analysis of photoinduced changes in the dielectric function}

Now we turn our discussion to the analysis of typical optical pump and $\mathrm{THz}$ probe measurements. In these experiments the electric-field waveform $E(t)$ of $\mathrm{THz}$ pulses transmitted through an unexcited sample and the pump-induced change in the THz waveform $\Delta E(t)$ are recorded. The latter is done by using a lock-in technique with modulation of the optical pump beam for each fixed delay time $\tau$ between the optical pump and $\mathrm{THz}$ probe pulse. In such experiments, we are interested in retrieving the photoinduced change $\Delta \epsilon(\nu)$ in the complex dielectric function $\epsilon(\nu)$ as a function of the frequency $\nu$. This can be achieved using the Fourier transform of the two waveforms $E(\nu)$ and $\Delta E(\nu)$ following a similar procedure as in Sec. II.D.1, where

$$
\frac{\Delta E(\nu)}{E(\nu)}=\frac{t(\epsilon+\Delta \epsilon, \nu)-t(\epsilon, \nu)}{t(\epsilon, \nu)} .
$$

Note that, for now, we consider the simple case of a quasistatic limit, in which the photoinduced change in the material properties occurs on a much longer time scale than the $\mathrm{THz}$ pulse duration. Such a quasistatic condition can be satisfied in a material with a long carrier lifetime and probed several picoseconds after pump excitation when the fast hot-carrier effects have abated.

We first consider the analysis for a homogeneously excited slab of material in vacuum of thickness $d$ [Fig. 2(a)] In the limit of a weak perturbation to the $\mathrm{THz}$ response, a linear relation between the pump-induced response and the ratio of the Fourier transform of the waveforms can be found, ignoring multiple reflections (Knoesel et al., 2004)

$$
\Delta \epsilon(\nu)=2 \epsilon(\nu)\left[i \sqrt{\epsilon(\nu)} \frac{2 \pi \nu d}{c}-\frac{\sqrt{\epsilon(\nu)}-1}{\sqrt{\epsilon(\nu)}+1}\right]^{-1} \frac{\Delta E(\nu)}{E(\nu)} .
$$

This expression includes propagation of the $\mathrm{THz}$ probe through the sample, as well as interfacial reflection losses at the boundary of the sample.

A useful approximation can also be applied when only a very thin region near the surface of a sample is excited [Fig. 2(b)]. This situation occurs when the pump light is strongly absorbed by a material, such as for above-bandgap photoexcitation of a semiconductor. It is convenient to approximate the excited region by a homogeneous region of width $l_{0}$, the penetration depth of the optical excitation in the sample. If the excitation density is sufficiently small, the phase accumulated by a $\mathrm{THz}$ wave propagating through the 


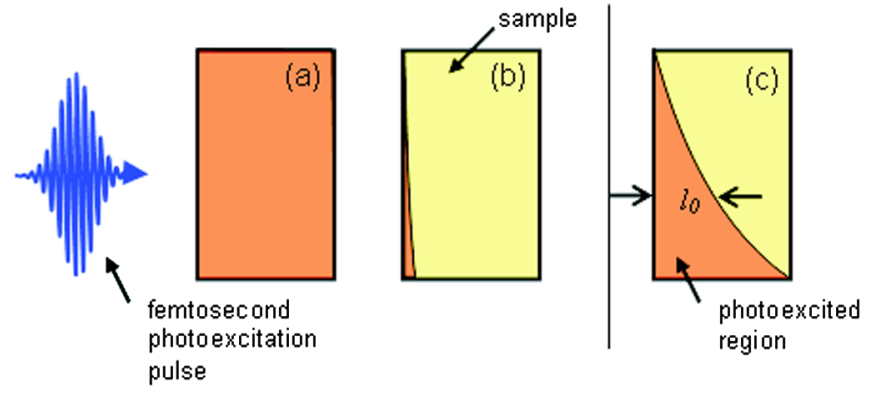

FIG. 2 (color online). Schematic of typical sample excitations achieved in an optical pump- $\mathrm{THz}$ probe experiment. Experiments with (a) homogeneous excitation and (b) interfacial excitation can be treated analytically.

excited region will be small (i.e., $\left.\sqrt{(\epsilon+\Delta \epsilon)} 2 \pi \nu l_{0} / c \ll 1\right)$. The exponential terms in the transmission function of the photoexcited region given by Eq. (6) can again be approximated by Taylor expansions. We thus obtain the following expression for the average change in the dielectric function inside the photoexcited region:

$$
\Delta \epsilon_{a v}(\nu)=-i \frac{c \sqrt{\epsilon(\nu)}}{\pi \nu l_{0}} \frac{\Delta E(\nu)}{E(\nu)} .
$$

Note that Eq. (7) follows directly from Eq. (6) under the neglect of the reflection contribution, which is not altered by photoexcitation for this case. Equation (7) applies to a broad range of $\mathrm{THz}$ pump-probe experiments where the condition of $\sqrt{(\epsilon+\Delta \epsilon)} 2 \pi \nu l_{0} / c \ll 1$ is fulfilled [see, e.g., (Hendry, Koeberg, O'Regan, and Bonn (2006) and Laarhoven et al. (2008)].

The analysis, however, is complicated when the penetration depth $l_{0}$ of the optical excitation in the material of interest is comparable to the wavelength of $\mathrm{THz}$ radiation. A numerical analysis is then required in which we divide the sample into many thin, homogeneous slabs, with different excitation densities. Similarly problematic is the effect of inhomogeneous excitation along the direction perpendicular to the propagation direction of the $\mathrm{THz}$ probe: finite difference time domain simulations are required (Kindt and Schmuttenmaer, 1999).

The discussion above applies to quasistatic changes in the material response. When the change in the material response occurs on a time scale comparable to or shorter than that of the $\mathrm{THz}$ pulse, one can no longer use the method described above for data analysis. This regime is typically encountered when probing immediately after optical excitation or in systems with very fast carrier recombination times. In these cases a complete two-dimensional scan of the pump-induced THz electric-field waveforms $\Delta E(t, \tau)$ at each optical pumpTHz delay time $\tau$ must be considered within an overall model of the material response, since the time-dependent frequencydomain material response function discussed above has no general physical meaning. In the limit of weak material response to the pump beam, the measurement can be considered as a particular form of four-wave mixing. More details can be found in Nemec et al. (2002).

\section{E. Typical responses observed}

\section{Drude response}

In bulk solids, the $\mathrm{THz}$ response of free carriers, whether from dopants or photoexcitation, can often be described with by a Drude response function. The result can be derived from several descriptions of charge motion within a solid, including the semiclassical Boltzmann transport equation and the Kubo-Greenwood analysis (Kubo, 1957; Hickey et al., 2001). The model, as discussed below, involves just a single relaxation parameter. Despite its simplicity, it has been verified for charge transport over a spectral range from $\mathrm{MHz}$ to the optical frequencies (Dressel and Scheffler, 2006) in various materials.

Heuristically, the Drude response can be derived from a simple semiclassical model of charge transport. In this picture, the charges are accelerated under the external electric field $E(t)$ according to an equation of motion of

$$
\frac{d^{2} \mathbf{r}}{d t^{2}}+\gamma \frac{d \mathbf{r}}{d t}=-\frac{e}{m} \mathbf{E}(t),
$$

where $r$ is the ensemble average of the displacement of the charge carriers, $\gamma$ denotes the damping rate or scattering rate, $e$ is the electronic charge, and $m$ is the effective mass of the charge carrier. For a given carrier density $N$ the dielectric response function $\epsilon_{D}$ to an alternating external electric field at angular frequency $\omega(=2 \pi \nu)$ can be solved from Eq. (8). This yields the well-known Drude response:

$$
\epsilon_{D}=\epsilon_{\infty}-\frac{\omega_{p}^{2}}{\omega^{2}+i \gamma \omega},
$$

where $\epsilon_{\infty}$ is the background dielectric constant.

The Drude response is characterized by a Lorentzian resonance centered at zero frequency with a linewidth $\gamma$, which is the charge carrier scattering rate; the inverse of the scattering time $\tau$. The amplitude of the dielectric response is determined by the plasma frequency $\omega_{p}$, defined as

$$
\omega_{p}^{2}=\frac{N e^{2}}{\epsilon_{0} m},
$$

with $N$ being the charge density and $\epsilon_{0}$ the permittivity of free space. The real part of $\epsilon_{D}$ crosses the zero point at the screened plasma frequency $\omega_{s p}=\omega_{p} / \sqrt{\epsilon_{\infty}}$. By plotting the inverse of the dielectric response $1 / \epsilon_{D}$, also known as the dielectric loss function, we find a peak centered at $\omega_{s p}$.

We can also write the Drude response in terms of the complex conductivity of the material

$$
\sigma_{d}=\frac{\sigma_{\mathrm{dc}}}{1-i \omega \tau}
$$

Here the parameter $\sigma_{\mathrm{dc}}=N e^{2} \tau / m$ gives the value of the dc conductivity of the material within the Drude model.

Depending on the relative value of the scattering rate and the $\mathrm{THz}$ frequency window, the observed Drude response will vary. Figure 3 shows the predicted dielectric function and complex conductivity for the case of a scattering rate within the $\mathrm{THz}$ frequency window and for a far higher scattering 

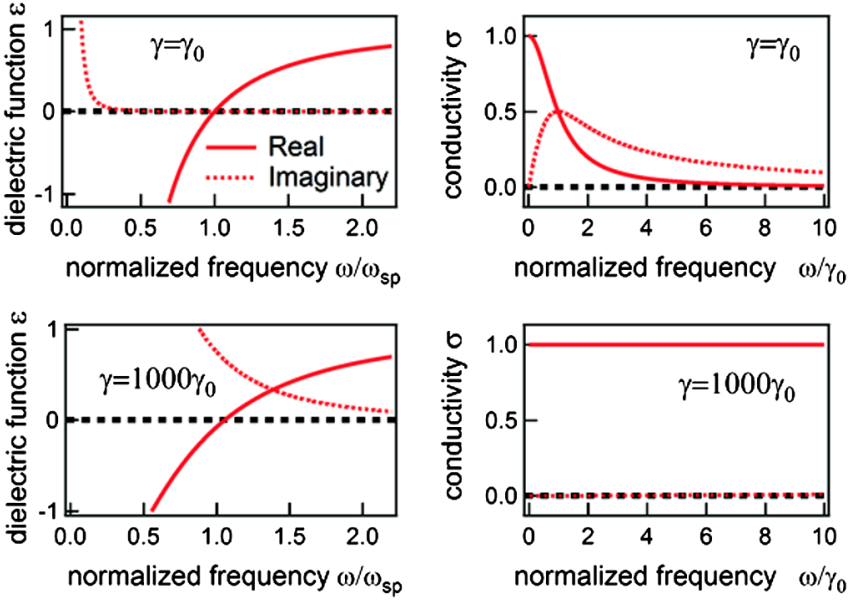

FIG. 3 (color online). Drude response for two regimes of scattering rates relative to the probe frequency window. Upper row: complex dielectric function and conductivity for a relatively low scattering rate $\gamma$; lower row: the same for a very high scattering rate. All solid (dotted) lines indicate real (imaginary) part of the response. The dotted lines indicate zero point. $\gamma_{0}$ is a frequency unit. The frequency axes of the conductivity plots are normalized for $\gamma_{0}$, while for the dielectric functions they are normalized for the screened plasma frequency $\omega_{s p}$ and $\epsilon_{\infty}$ is set to 1 .

rate. When the scattering rate is within the $\mathrm{THz}$ probe window, both the real and imaginary parts of the response are finite and dispersive. In this case, we can determine both of the parameters in the Drude model, i.e., the plasma frequency and the scattering rate. If the carrier mass is known, this analysis then provides the carrier concentration $N$ from the plasma frequency and the carrier mobility $\mu=e / m \gamma$ from the scattering rate. When the scattering rate far exceeds the available $\mathrm{THz}$ frequency range, then the analysis can only yield the dc conductivity $\sigma_{\mathrm{dc}}$. In this limit, the real part of the conductivity is finite, while the imaginary part is negligible. This corresponds to a purely imaginary dielectric function, with a trivial $1 / \omega$ frequency dependence. For this case, one consequently cannot independently determine the carrier concentration and scattering time. In many experiments involving photogenerated carriers, the excitation conditions provide information on the carrier concentration. The $\mathrm{THz}$ data within the Drude model are then seen to provide the carrier mobility (or scattering rate, assuming existing knowledge of the carrier effective mass).

Charge carriers in many semiconductors exhibit scattering times of a few hundred femtoseconds, corresponding to linewidths of the Drude peak of around $1 \mathrm{THz}$. Such a response is therefore readily characterized by $\mathrm{THz}$ pulses generated with either photoconductive antennas or ZnTe crystals, which allow one to determine the real and imaginary components of the dielectric response over a broad spectral window exceeding $1 \mathrm{THz}$. Hence, when the Drude model is applicable, $\mathrm{THz}$ spectroscopy can be used to simultaneously characterize the two key parameters associated with electrical conductivity, the plasma frequency and carrier scattering rate. Values for the carrier mobility $\mu$ and the charge concentration $N$ then follow immediately if the effective mass of the carriers is known.

\section{Lorentzian oscillator}

In addition to the free-carrier response described above, a second distinct type of $\mathrm{THz}$ response frequently observed is that with a resonance at a finite frequency. The $\mathrm{THz}$ spectral regime covers many fundamental excitations including rotations, vibrations, and low-lying electronic transitions in molecules and collective modes in condensed matter such as phonons, plasma, magnons, and energy gaps associated with superconductivity (Averitt and Taylor, 2002; Kaindl et al., 2006) as well as intraexcitonic transitions for excitons (Kira et al., 2004; Koch et al., 2006). A response with a resonance at finite frequencies, as will be shown in the remainder of this review, also occurs for a number of other situations, including the effective medium-type response (Garnett, 1904; Stroud, 1975; Choy, 1999), the plasmon-type response (Nienhuys and Sundstrom, 2005a), the Drude-Smith response (Smith, 2001), and the response associated with "dispersive" transport (Dyre and Schroder, 2000).

Here we present the simplest description of response at a finite frequency, the Lorentz oscillator (given by the scattering rate). This is a direct generalization of the Drude model including a response a a finite frequency and follows from the generalization of Eq. (8) to include a restoring force. The dielectric function thus can be described by

$$
\epsilon=\epsilon_{\infty}+\frac{A}{\omega_{0}^{2}-\omega^{2}-i \omega \gamma}
$$
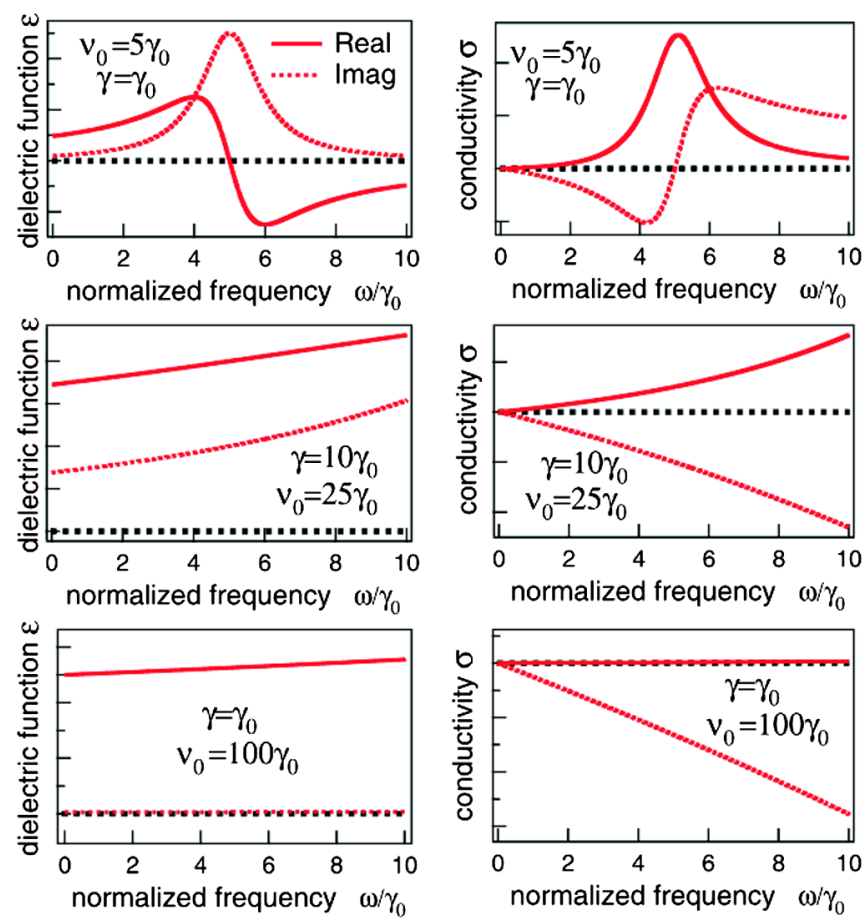

FIG. 4 (color online). Resonant response with different line widths and resonance frequencies relative to the probe frequency window. Upper row: complex dielectric response and conductivity for fully resonant response; middle row: the low-frequency side of the resonance; lower row: completely off-resonant response. All solid (dotted) lines indicate real (imaginary) part of the response. The dotted lines indicate zero point. $\gamma_{0}$ is a frequency unit. All frequency axes are normalized for $\gamma_{0}$. The dielectric response has been displaced by $-\epsilon_{\infty}$ for clarity. 
where $A$ is the amplitude, $\omega_{0}$ denotes the resonance frequency, and $\gamma$ defines the width of the resonance. Figure 4 shows the corresponding response as measured over a finite spectral range for different parameters. Applications of this treatment will be discussed below.

\section{F. Comparison to conventional transient photoconductivity}

Optical pump-THz probe spectroscopy has emerged as a powerful technique for probing charge transport in a variety of homogeneous and inhomogeneous materials. It is therefore useful to compare this technique with the conventional transient photoconductivity measurements, such as time-of-flight method (Bos and Burland, 1987; Lee et al., 1994; Moses et al., 1997; Konenkamp et al., 1999). In these measurements a transient current induced by a static electric field is measured as a function of time after photoexcitation by a short optical pulse. In this section, we briefly discuss the similarities and differences between these different experimental techniques.

In conventional transient photoconductivity measurements, the temporal evolution of the photoinduced current is determined by two factors: the density of mobile charge carriers induced by the photoexcitation and their response to the static external electric field. In Fig. 5(a) we show a simple time dependence of the transient current. Here the response of the photoexcited electrons is assumed to follow the Drude model with a scattering rate $\gamma_{0}$ and the population of the conduction electrons is described by a step function at time zero. This is representative of photoexcitaion in a bulk crystalline semiconductor by an ultrafast laser pulse. The transient current, calculated according to Hendry et al. (2005), is seen to rise exponentially with a time constant determined by the electron scattering rate. Thus, the time evolution of the current has a
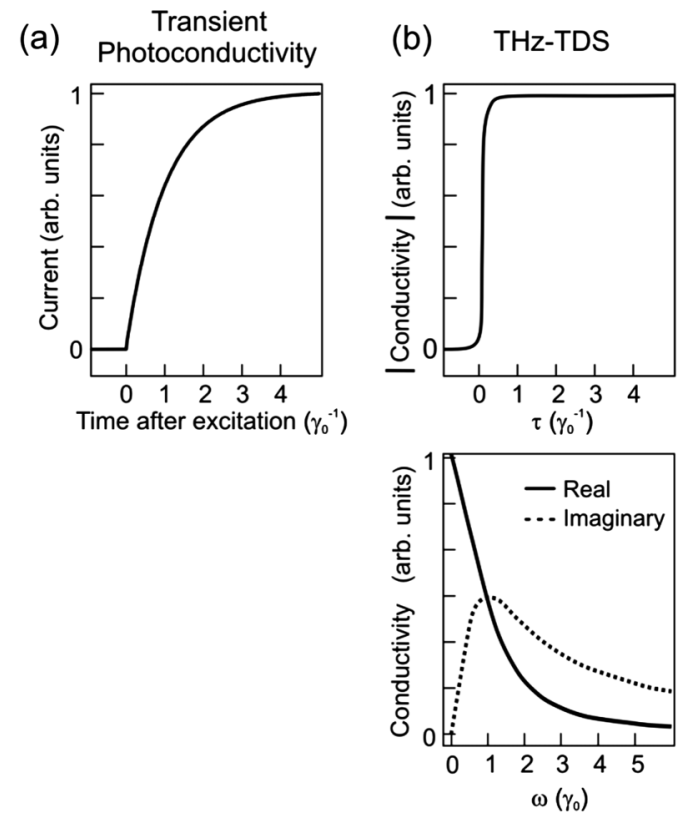

FIG. 5. Time dependence of signals in a (a) transient photoconductivity measurement and (b) THz-TDS measurement, both for a Drude electron gas undergoing a step increase in density at time zero. signature of the type of photospecies, and the transport mechanisms and population dynamics in the material of study. A more complicated time dependence of photoconductivity is expected in cases where charge localization and hopping transport mechanisms (Scher and Montroll, 1975; Bos and Burland, 1987; Konenkamp et al., 1999), excitonic effects (Lee et al., 1994; Moses et al., 1997), or population dynamics (Orenstein and Kastner, 1981) are important.

In a typical optical pump-THz probe experiment, an optical pump pulse creates an excitation in a sample, which is probed with a picosecond $\mathrm{THz}$ pulse at a time $\tau$ after the excitation. In this case, one probes the time-dependent conductivity of a material using a transient field $E(t)$ rather than a static one. Generally this is a two-dimensional detection of the THz probe electric field at time $(t)$ and time $(\tau)$ after the photoexcitation. It is more straightforward to present this information in terms of a time and frequency-dependent conductivity $\sigma(\omega, \tau)$, i.e., a frequency-dependent conductivity which depends parametrically on time $\tau$ after photoexcitation. If $\sigma(\omega, \tau)$ changes fairly slowly with time during the $\mathrm{THz}$ probe pulse (typically $\sim 1 \mathrm{ps}$ ), one can define a quasisteady-state conductivity $\sigma(\omega)$ (see previous section) that reflects only the dielectric response of the material (Fig. 5). The population dynamics is generally revealed independently of the dependence on $\tau$ as shown in Fig. 5(b)]. This is one of the major differences between THz-TDS measurements and conventional photoconductivity measurements: for the latter, both the response of the photoexcited species and their timedependent populations manifest themselves in the transient current as a function of time after photoexcitation. It should be noted that the general case, where $\sigma(\omega, \tau)$ varies on a time scale that is shorter than the $\mathrm{THz}$ pulse duration, such a simple separation does not apply and the temporal evolution of the $\mathrm{THz}$ fields may be quite complex (Kindt and Schmuttenmaer, 1999; Nemec et al., 2002; Hendry et al., 2005; Nemec, F. Kadlec, C. Kadlec et al., 2005; Nemec, Kadlec, Surendran et al., 2005; Nienhuys and Sundstrom, 2005b; Schins et al., 2007; Schins, 2010), as different temporal segments of the $\mathrm{THz}$ pulses probe different sample properties.

The pulsed nature of the THz electric field in a THz-TDS measurement also has implications regarding the length scales over which conductivity is probed. For example, a simple estimate of the thermal velocity of an electron at room temperature $\left(\sim 10^{5} \mathrm{~m} / \mathrm{s}\right)$ indicates that, in the $\sim 1 \mathrm{ps}$ duration of a $\mathrm{THz}$ cycle, the motion of charges is probed only on a length scale of $<100 \mathrm{~nm}$. Owing to the short time and length scales over which the $\mathrm{THz}$ conductivity is determined, the $\mathrm{THz}$ conductivity is relatively insensitive to defect-related effects that impede carrier motion over large distances. As such, charge mobilities inferred from $\mathrm{THz}$ measurements therefore usually represent intrinsic, upper limits for electron transport (Hendry, Koeberg, Schins et al., 2006b).

\section{CHARGE CARRIERS IN BULK SEMICONDUCTORS}

In this section, we illustrate the capabilities and achievements of $\mathrm{THz}$ spectroscopy in characterizing charge carrier dynamics of both free carriers (Sec. III.A) and excitons (Sec. III.B). 


\section{A. Free carriers}

The particular attractiveness of $\mathrm{THz}$ spectroscopy as a probe of charge carriers in semiconductors originates from the fact that the dielectric response of charge carriers is strong and dispersive in the $\mathrm{THz}$ region of the electromagnetic spectrum. Conversely, one can characterize the nature and mobility of charge carriers readily by probing the dielectric response using $\mathrm{THz}$ radiation. As we will show in this section, this allows for the direct determination of key conductivity parameters and their time evolution after photogeneration. Sections III.A.1, III.A.2, and III.A.3 deal with measurements of transport properties in inorganic and organic semiconductors. In Sec. III.A.4 ultrafast dynamics of charge carrier generation and decay are discussed.

\section{Determination of scattering times and plasma frequencies in bulk semiconductors}

THz-TDS allows one to measure both the amplitude and phase (or, equivalently, both the real and imaginary parts) of the material response over a wide range of frequencies. Frequently, the mobility of electrons exceeds that of the holes, and the data can be described by the Drude model with one carrier type. Hence the two key conduction parameters, the plasma frequency and scattering rate, (Beard et al., 2000; Hendry, Wang, Shan et al., 2004; Hendry, Koeberg, Pijpers, and Bonn, 2007; ), can be determined by simultaneously fitting both the real and imaginary parts of the frequency dependence of the conductivity.

In practice, however, the finite range of probe frequencies, determined by the bandwidth of the spectrometer, can set limitations. For example, for a Drude model conductor, if the charge scattering rate lies well outside the probe frequency range, the phase of the conductivity is approximately zero across the spectrum, and one can obtain reasonable fits to experimental data with a wide range of Drude parameters (Hendry, Wang, Shan et al., 2004). This limitation can be overcome by determining, in addition, the density of absorbed photons in the sample. This allows a method of independently determining the plasma frequency (assuming that the carrier mass is known), so that the $\mathrm{THz}$ spectrum can be fitted by the Drude model with only one adjustable parameter: the scattering rate. A similar approach is useful for samples with more than one photoexcitation species contributing to the $\mathrm{THz}$ spectrum, helping to separate carrier mobility from density.

This ability of $\mathrm{THz}$ spectroscopy to independently determine both scattering rates and plasma frequencies has proven particularly useful in recent work that assessed the efficiency of impact ionization processes in the low-band gap semiconductors $\mathrm{PbS}$ and $\mathrm{PbSe}$ (Pijpers et al., 2009). Impact ionization refers to a mechanism in which a photon possessing an energy of several times the band gap is able to convert its excess energy into the generation of more than one electron-hole pair. The terahertz response was measured following optical excitation at photon energies ranging from the infrared (close to band gap) to the ultraviolet. By verifying the uniformity of the scattering rate for all pump wavelengths and fluences, one is assured that the plasma frequency, and hence the signal amplitude, is directly

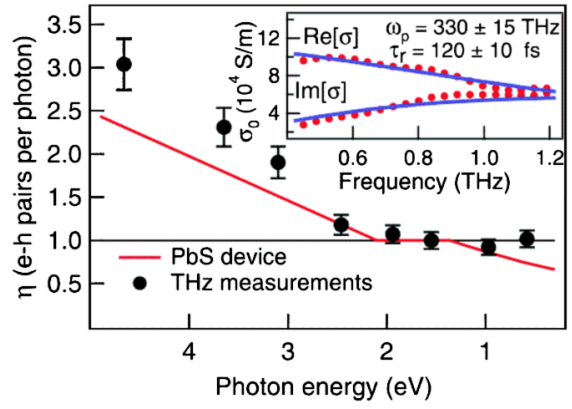

FIG. 6 (color online). Number of generated electron-hole pairs per photon in bulk $\mathrm{PbS}\left(E_{\text {gap }}=0.42 \mathrm{eV}\right)$ as a function of photon energy (circles). Also shown is the results of a device measurement (sloping solid line) from Smith and Dutton (1958). The inset shows the conductivity and the fit to the Drude model and corresponding plasma frequency and scattering time. From Pijpers et al. (2009).

proportional to the charge carrier density. The number density of absorbed excitation photons can be determined separately. Combining this quantity with the photogenerated charge carrier density inferred from terahertz measurements, one can directly determine the efficiency of impact ionization, measured in electron-hole pairs per photon. The results for bulk $\mathrm{PbS}\left(E_{\text {gap }}=0.42 \mathrm{eV}\right)$ are shown in Fig. 6 as a function of the photon energy of the excitation. It is apparent from this figure that the impact ionization efficiency is rather low (it takes a photon energy of $\sim 7 E_{\text {gap }}$ to produce two, rather than one, electron-hole pairs). However, compared to the efficiency of this process in quantum dots (QDs), the impact ionization efficiency is larger in bulk materials than in QDs for a given photon energy. This is in contrast to previous expectations that quantum confinement would lead to an enhancement of impact ionization.

It is interesting to compare the $\mathrm{THz}$ results to device measurements, shown as the solid sloping line in Fig. 6 (Smith and Dutton, 1958). Contrary to the $\mathrm{THz}$ measurements, device measurements to determine impact ionization efficiencies rely on photocurrent measurements (Brinza et al., 2005), which require charges to move over large distances on long time scales. As a result, recombination losses such as Auger recombination and trapping at surface defects can introduce uncertainties in the assessment of inherent carrier multiplication efficiencies in devices. In $\mathrm{THz}$ studies, the carrier density is determined picoseconds after photoexcitation, thus excluding the influence of all but the fastest trapping and recombination processes.

\section{Limitations of the Drude model}

Although the Drude model has been successfully applied in many instances where the frequency-dependent conduction was determined, there are several examples in which a description of the electron response requires more refined models.

For instance, the conductivity of $n$-type and $p$-type doped silicon samples at low charge densities departs from the Drude form in the $\mathrm{THz}$ spectral range. The data can, however, be fit by the Cole-Davidson model (Jeon and Grischkowsky, 1997; Jeon and Grischkowsky, 1998): 


$$
\sigma=\frac{\sigma_{\mathrm{dc}}}{(1-i \omega \tau)^{\beta}}
$$

where the parameter $\beta \neq 1$ describes the deviation from the Drude behavior and can be considered a measure of the departure from a carrier response characterized by a single scattering time (see Fig. 7). For low doping densities, the data could be described with $\beta \approx 0.8$. Only at charge densities above $10^{17} \mathrm{~cm}^{-3}$, a fit with $\beta=1$ was obtained, hence recovering the Drude model.

A second example where the Drude model should be handled with care is for very high charge densities. In these regimes scattering events between electrons and holes can no longer be neglected, and the scattering time and hence the mobility decrease with increasing charge density. This effect is most pronounced in materials with a small (real) dielectric constant $\epsilon^{\prime}$ since charge carriers are less effectively screened from each other. For instance, in silicon (small $\epsilon^{\prime}$ ) a hundredfold higher charge density from $10^{21}$ to $10^{23} \mathrm{~m}^{-3}$ leads to a reduction of the scattering time by roughly the same amount, while in titanium dioxide (large $\epsilon^{\prime}$ ) the same increase has barely any effect on $\tau$ (Hendry, Koeberg, Pijpers, and Bonn, 2007). We note that, even when electron-hole interactions become important, the dispersion of the conductivity may still appear Drude-like; the Drude parameters will be modified, however.

Another limitation in the applicability of the Drude model can be found in the limit of very strong electron-phonon interactions. For relatively weak electron-phonon interactions, one can consider electrons to exhibit their band mass, but to scatter by emission of absorption of a phonon. As the electron-phonon interaction becomes strong, it becomes appropriate to consider a local deformation of the lattice around a charge carrier. The free electron approximation basically assumes a rigid lattice. In reality the electric field exerted by a charge carrier displaces the lattice ions in its vicinity, forming a polarization field around it. The charge carrier and its accompanying lattice deformation result in the formation of a quasiparticle, the polaron (Devreese and Peeters, 1984; Klingshirn, 2006). The degree of coupling scales with the lattice polarizability and is measured by the Fröhlich constant

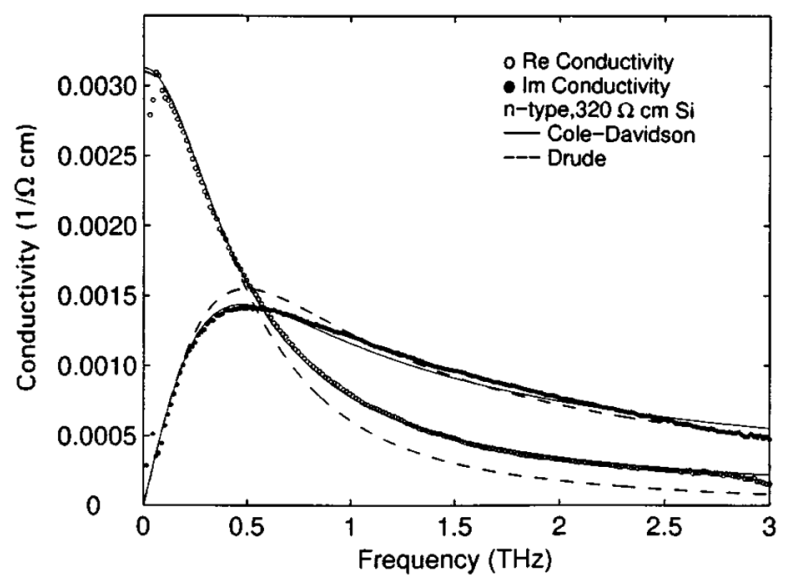

FIG. 7. Real and imaginary parts of the conductivity of an $n$-doped $\mathrm{Si}$ wafer with fits to the Cole-Davidson model (solid line) and the Drude model (dashed line). From Jeon and Grischkowsky (1998). $\alpha$, which characterizes the electron-phonon coupling energy in terms of the phonon energy (Frohlich et al., 1950). The potential well created by the polarization field hinders the movement of the polaron since it has to "drag" the field along. This results in an increased effective mass of the carriers, termed polaron mass. For sufficiently weak coupling ( $\alpha \ll 6$ ), the wave function of the carrier remains extended (large polaron) and coupling merely increases the polaron mass, but leaves its transport Drude-like in nature, as THzTDS measurements on sapphire have shown (Shan et al., 2003). For $\alpha \gg 6$, the potential well is deep enough to localize the carrier wave function, i.e., trap the charge (small polaron formation). In this limit transport can only occur via tunneling or hopping and the conductivity is not expected to follow Drude behavior.

Hence, charge carrier transport can in principle be divided into two idealized cases: coherent, bandlike transport in delocalized states when intersite coupling is large and incoherent hopping transport from one localized state to another, characterized by low intersite coupling. In addition to different types of response (Drude-like versus non-Drude-like), a second way to distinguish the two types of response is through the temperature dependence of the charge carrier mobility. While the mobility is generally thermally activated for hopping processes, i.e., it increases with temperature, it generally decreases with temperature in bandlike transport due to increased carrier scattering with phonons at elevated temperatures. In many complex materials this distinction might be an oversimplification since several types of charge transport mechanisms can occur on different time and length scales in a single material. This is particularly true for organic semiconductors where one often has to distinguish between intramolecular and intermolecular transport, both of which are strongly influenced by the sample morphology. Given the surge in recent applications of THz TDS on these systems, we will elaborate on these issues in more detail.

\section{Organic semiconductors}

The organic semiconductors are commonly divided into two classes: (i) small molecules that are typically deposited as crystalline material by evaporation and (ii) semiconducting organic polymers that are processed in solution. Smallmolecule semiconductors like acenes are typically characterized by an extended $\pi$ system along the molecular backbone on which charge carriers are delocalized. Under optimized process conditions they can assemble into crystals, bonded by intermolecular van der Waals forces. The key to efficient intermolecular electronic coupling lies in an optimum overlap between the $\pi$ orbitals of adjacent molecules. Several $\mathrm{THz}$ studies have been performed on single crystals of pentacene (Thorsmolle et al., 2004) and functionalized pentacene (Hegmann et al., 2002), as well as on thin films of both materials (Ostroverkhova et al., 2005). The photoinduced conductivity is real and essentially independent of frequency in the THz range Fig. 8. This can in principle be described by the Drude model with scattering times of only a few femtoseconds. Such a low scattering time would correspond to a carrier mean free path on the order of the intermolecular distance, indicating that charge carriers are likely to be localized on the molecule. 


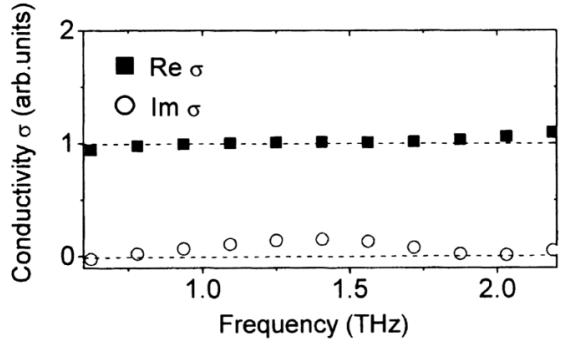

FIG. 8. Conductivity of an organic crystal pentacene at room temperature; the dashed lines are guides to the eye for a flat, frequency-independent response. From Ostroverkhova et al. (2005).

For single crystals, the photoconductivity drops within 2 ps and subsequently decays on longer time scales following a power-law decay $\sim t^{-\beta}$ with $\beta=0.5$. This was originally interpreted as the onset of dispersive (e.g., hopping) transport found in disordered media, pointing to localized charge carriers. While material dispersion can manifest itself in a time dependence (after photoexcitation) of the dc conductivity, in THz-TDS material polarization is normally expressed in the frequency dependence of the conductivity, while population dynamics will generally present themselves as a function of time after photoexcitation (see Sec. II.F). Moreover, the mobility of all measured samples, single crystals and thin films, was found to decrease with increasing temperature, which would indicate bandlike transport.

A possible explanation for this apparent contradiction was offered by Troisi and Orlandi (2006). Since the rate-limiting transport step is the intermolecular charge transfer, which in turn depends crucially on the distance between molecules, a so-called nonlocal (Peierls) electron-phonon coupling effect was proposed. In essence, it predicts a time-dependent modulation of the intermolecular coupling by the molecular vibrations of the molecules in the crystal that is sufficiently strong to localize carriers on the molecule and yet yields a bandlike temperature dependence of the mobility.

This was directly verified in a terahertz experiment on pentacene where on charge injection a pronounced absorption peak at $1.1 \mathrm{THz}$ was observed (Laarhoven et al., 2008). This peak was attributed to a charge-induced activation of a soft vibrational mode at that frequency that couples strongly to the charge carrier dynamics. In other words, the intermolecular distance is modulated at a frequency of $1.1 \mathrm{THz}$ and so is the intermolecular electronic coupling.

Another class of organic semiconductors can be found in semiconducting polymers, which are characterized by delocalized $\pi$-electron systems along the polymer backbone. The electronic properties of polymer thin films are generally inferior compared to their small-molecule counterparts. The morphology lacks the long-range order that characterizes polycrystalline thin films and especially single crystals of small molecules. The electronic coupling between polymers is hence comparably weak, resulting in a bottleneck for carrier transport, which leads to lower macroscopic charge carrier mobilities. The intrachain mobility, however, is anticipated to be high, since charge carriers are, at least in absence of torsional disorder originating from kinks, expected to be delocalized on the conjugated backbone.

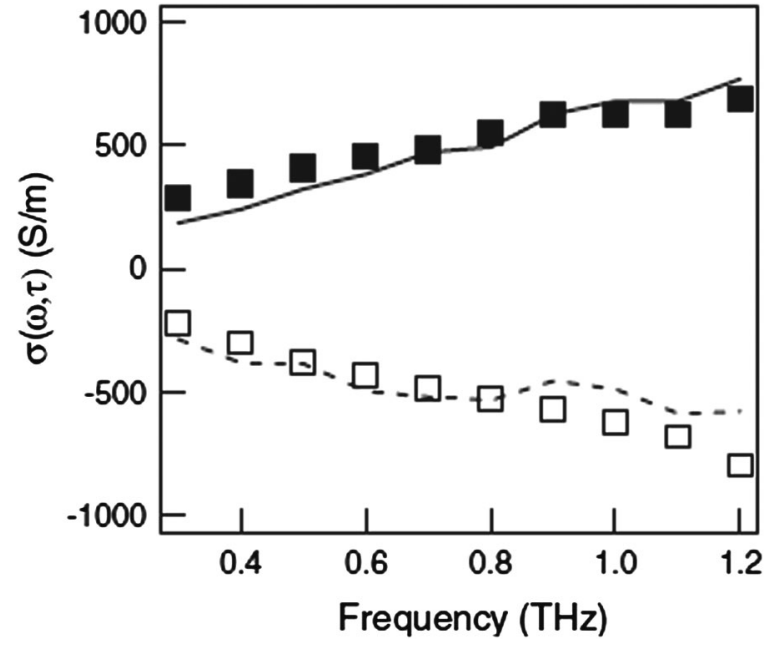

FIG. 9. Real conductivity (filled squares) and imaginary conductivity (open squares) of an MEH-PPV/PCBM blended sample, measured $10 \mathrm{ps}$ after photoexcitation. Lines are the results of model calculations. From Hendry, Schins et al. (2004).

Techniques that predominantly probe charge transport on the molecule, i.e., are only sensitive to charge carrier displacements on the nanometer scale, are therefore desirable in order to gain access to this regime. This problem can be translated into time by stating that such a technique ought to be sensitive to charge carrier movements on picosecond time scales, i.e., operating at $\mathrm{GHz}$ or $\mathrm{THz}$ frequencies. Indeed, microwave measurements at $34 \mathrm{GHz}$ on ladder-type poly(p-phenylenes) revealed that charge transport along the backbone that is only obstructed by the chain ends would result in mobilities as high as $600 \mathrm{~cm}^{2} / \mathrm{Vs}$ (Prins et al., 2006). THz TDS can therefore be expected to deliver similar results and with it the time-resolved evolution of photoexcited species.

A typical photoconductivity spectrum for semiconducting polymers, in this case from a MEH-PPV thin film, is shown in Fig. 9. The existence of a real part of the conductivity indicates the presence of free charge carriers. However, contrary to the Drude model, the real part decreases with decreasing frequency, approaching very low values close to zero frequency. This indicates that the long-range transport at dc frequencies is poor. The imaginary part differs from the Drude response as well, being negative and increasing with frequency. This type of behavior is characteristic of localized charge carriers in disordered systems. In these types of systems lacking long-range order, a highly corrugated potential energy landscape exists for carrier motion. Even along the backbone of one polymer strand, the mobility will be hindered by interruption of the $\pi$-electron continuum caused, for instance, by chemical or torsional defects. At increasingly high frequencies of the terahertz field, the mobility will be probed over increasingly short length scales. Hence, at high frequencies, the carrier response is probed in regions of high mobility.

The complex conductivity is thus characteristic of dispersive free charge transport in a disordered medium, where localization caused by the disorder in the material structure causes non-Drude behavior. Indeed, the observed $\mathrm{THz}$ 
conductivity spectrum could be reproduced very well using a model by Grozema et al. (2002) to describe this frequency dependence. This model simulates the conductivity of a charge along a polymer chain. It is based on the tight-binding approximation combined with static torsional disorder deviations from planar alignment of the chain determining the effective conjugation length of the polymer chain.

From these initial terahertz conductivity measurements on semiconducting polymers, it is apparent that there is an important effect due to the morphology of the polymer chains. It was subsequently shown that interactions between polymer chains also affect the photoconductivity of these materials: in a dropcast film of semiconducting polymers, the close proximity of nearest-neighbor chains facilitates exciton dissociation on subpicosecond time scales by allowing the resulting electron and hole charges to escape on separate chains and permitting significant real conductivity on much longer time scales. In solution, where polymer chains interact to a much lesser extent, significantly fewer charges are photogenerated, but the increased freedom of the polymer chains results in a larger conjugation length and an exciton with greater spatial extent and corresponding larger average polarizability than in the film sample (Hendry et al., 2005). More systematic studies of morphology issues, combining $\mathrm{THz}$ results with thin-film transistor device measurements, have revealed the influence of conjugation effects, molecular weight and film deposition conditions on the charge carrier mobility (Esenturk, Kline et al., 2008; Esenturk, Melinger, and Heilweil, 2008).

Apart from pristine polymers, films blended with fullerene derivatives [usually phenyl-C61-butyric acid methyl ester (PCBM)] have been investigated as well (Hendry, Schins et al., 2004; Ai et al., 2006; Cunningham and Hayden, 2008; Nemec et al., 2008; Parkinson et al., 2008; Nemec, Kuzel, and Sundstrom, 2009). The inclusion of PCBM is primarily intended for the application in organic solar cells; they provide a means to dissociate excitons after photoexcitation by acting as electron scavengers. Accordingly, free-carrier yields between a few and 30 times the yield in pristine films have been reported. Additionally, in blends a large fraction of free carriers is still present after nanoseconds, corroborating efficient spatial electron-hole separation (Parkinson et al., 2008). Two studies on poly(3-hexylthiophene) (P3HT)-PCBM blends found that photoexcitation below the band gap with $800 \mathrm{~nm}$ wavelength yielded comparable quantum efficiencies for charge generation and similar decay dynamics as compared to excitation at or above the band gap (Cunningham and Hayden, 2008; Parkinson et al., 2008). The origin for this behavior is still unclear. It was suggested that a charge-transfer state that is present in blends but absent in pristine polymers might act as an intermediate state for free-carrier generation.

Recent work has employed Monte Carlo simulations to explain measurements on fullerene blends (Nemec, Nienhuys et al., 2009). The application of Monte Carlo simulations seems a promising, albeit complex, route to take in order to interpret terahertz spectra in complex material systems such as semiconducting polymers. These simulations can be adapted to the specific morphological conditions and include different types of dynamics. The results hence offer important microscopic insights that more general and phenomenological models simply cannot provide.
It is apparent that the spectral signatures associated with conductivities of delocalized and localized charge carriers are different in the terahertz regime-and their differentiation thus possible using $\mathrm{THz}$ spectroscopy. This feature is particularly helpful for the characterization of materials that fall into the intermediate regime between large and small polarons. Measurements on these materials using other techniques are often inconclusive about the nature of charge transport. As an example, in titanium dioxide terahertz measurements revealed that, despite a large extracted Fröhlich constant of six, charge carriers were still delocalized (Hendry, Wang, Shan et al., 2004), contradicting previous reports that claimed the presence of small polarons. These examples demonstrate that $\mathrm{THz}$ TDS provides a unique tool to investigate photoconductivity-following the response to photoexcitation over a large frequency window. These insights, combined with complementary techniques that are sensitive to longer time and length scales, make a valuable contribution to developing an overall picture of photoconductivity in a variety of materials. In the following section, we discuss another appealing feature of THz TDS: the possibility to determine photoconductivity at different times after photoexcitation, with very high time resolution.

\section{Ultrafast dynamics}

Unlike other ultrafast techniques such as luminescence and transient absorption, THz TDS is applicable to the study of carrier dynamics in indirect-gap semiconductors (Koch et al., 2006). Moreover, other ultrafast techniques are usually sensitive to either the sum or the product of the electron and hole population densities and do not measure the conductivity directly, while methods which are sensitive to the diffusive motion of carriers, such as transient grating or four-wave mixing, are not able to determine the conductivity on very fast time scales (Othonos, 1998; Chemla and Shah, 2001; Axt and Kuhn, 2004; Cundiff, 2008). THz TDS is therefore an extremely useful tool for determining material conductivities on very fast time scales.

The first THz-TDS measurements were performed on impurity-doped semiconductors (Vanexter and Grischkowsky, 1990; Katzenellenbogen and Grischkowsky, 1992). The emergence of amplified Ti:sapphire systems in the 1990s (Backus et al., 1998) eventually provided the possibility to readily create charge carriers by optical means. Not only did this offer greater flexibility in generating different charge carrier densities, but it also permitted the study of systems driven out of equilibrium by optical excitation processes and to monitor the evolution (Elsaesser and Woerner, 1999; Shah, 1999). This is particularly useful for the determination of the mobility in systems that possess a high density of trap states or fast electron-hole recombination channels, for instance. Traditional techniques like time-offlight or photocurrent spectroscopy measure the dc current after photoexcitation (Brinza et al., 2005). Naturally, the carriers have to travel significant distances to reach the electrodes - too long for some materials to collect them all. THz TDS can take conductivity "snapshots" before trapping or recombination mechanisms set in. These are not influenced by processes occurring after distances much larger than the carrier mean free path which is typically less than $100 \mathrm{~nm}$. 
Mobilities obtained with THz-TDS measurements can therefore be considered as an upper limit for semiconductors and semiconductor devices. From the observed dynamics, trapping processes occurring on picosecond time scales can be identified (Jepsen et al., 2001; Messner et al., 2001). Especially in materials that possess a low degree of crystallinity or porous materials, the conduction pathway can be significantly obstructed, resulting in the localization of charge carriers. Terahertz measurements on such materials will be discussed in Sec. IV.A. Dynamics in the sub-ps regime are within reach as well. Probe pulses in the few-THz range allow under certain circumstances time resolutions on the order of 100 s of fs. This permits for instance the study of intraband dynamics of charge carriers driven out of their equilibrium. With state-of-the-art lasers generating midinfrared probe pulses the probe spectrum not only covers carrier responses but vibrational modes of the lattice as well and the time resolution can be as high as 10 fs (Huber et al., 2000; Sell, Scheu et al., 2008). This gives access to the dynamics of quasiparticle correlations like the coupling between charge carriers and phonons, as will be shown below.

a. Intraband dynamics The first $\mathrm{THz}$ studies of intraband relaxation dynamics were carried out in model semiconducting materials like GaAs. While THz transmission studies of GaAs were carried out as far back as in the early 1990s (Greene et al., 1991), the first truly ultrafast THz study of the relaxation processes immediately following photoexcitation was carried out by Beard et al. (2000). In this pioneering work, they showed that by deconvolving the detector response function one can obtain information about material conduction on time scales down to $\sim 200$ fs after photoexcitation, while accurately determining the carrier dynamics and mobilities.

The photoexcitation energies used by Beard et al. (2000) were high enough to excite carriers into adjacent valleys in the band structure of GaAs (labeled $L$ and $X$ valleys). Since the conduction electrons in either the $L$ or $X$ valley have a much lower mobility than electrons in the main valley $(\Gamma)$, the measured dynamics reflected the electrons returning to the $\Gamma$ valley. While the conductivity on long time scales was found to be well described by the Drude model, with good agreement with literature values for the Drude parameters, they uncovered a contribution to the frequency-dependent conductivity on time scales (from 0.2 to 3 ps) after photoexcitation that had not been previously observed. This was essentially described by a time-dependent scattering rate (see Fig. 10), which was attributed to the influence of phonon dynamics on intraband free-carrier absorption. At early times phonons can be either absorbed or emitted to assist the THz photoabsorption [inset (a)], whereas at long delay times, after the carriers have relaxed to the bottom of the conduction band, their energy is not great enough to allow emission of a phonon to accompany photoabsorption [inset (b)], corresponding to a reduced scattering rate as shown in Fig. 10. This was also predicted earlier by Vengurlekar and Jha (1991).

In this example hot carriers received the required kinetic energy to scatter into the $L$ or $X$ valley by the excess energy of the optical pump. It is also possible to induce intervalley scattering through the application of an electric field. Charge carriers then get accelerated until their kinetic energy is

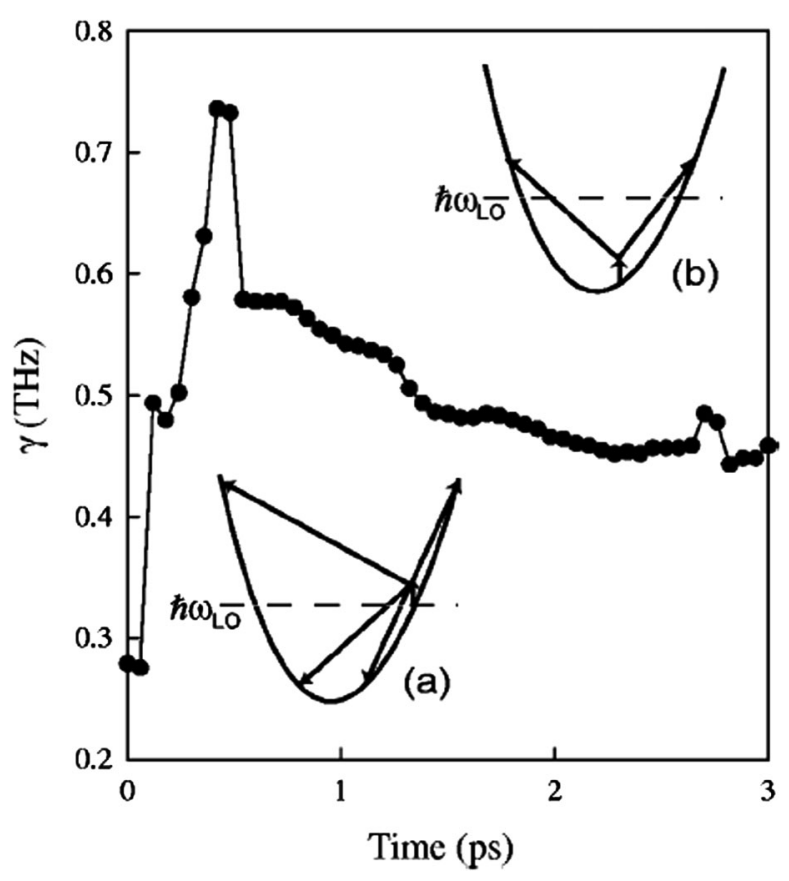

FIG. 10. Plot of the scattering rate $\gamma$ as a function of time after photogeneration of carriers in GaAs (corresponding to the frequency of the peak of the imaginary part of the conductivity). Variations as a function of time are attributed to phonon-assisted intravalley relaxation processes. Inset (a) shows the $\mathrm{THz}$ absorption assisted by LO-phonon absorption and emission. Inset (b) shows that after the electron distribution has relaxed below the longitudinal optical phonon frequency, $\omega_{\mathrm{LO}}, \mathrm{THz}$ absorption is only assisted by LOphonon absorption. From Beard et al. (2000).

sufficient to scatter into another valley. $\mathrm{THz}$ measurements on a GaAs wafer that was subjected to a dc field showed a decrease in conductivity for increasing electric fields and for field strengths above $3 \mathrm{kV} / \mathrm{cm}$ (Zhou et al., 2008). The decrease was attributed to an increasing amount of charge carriers that have scattered into the $\mathrm{L}$ valley and hence exhibit a lower mobility.

The recent development of high-energy single-cycle pulses in the low-THz regime as mentioned in Sec. II.A.3 has provided a unique tool to study such hot-carrier effects with high time resolution. Currently, electric fields of several hundred $\mathrm{kV} / \mathrm{cm}$ for pulses with a spectral coverage from 0.1 to $3 \mathrm{THz}$ have been realized (Blanchard et al., 2007; Hebling, Yeh, Hoffman, Bartal, and Nelson, 2008). Viewed in the time domain such frequencies correspond to electric fields that retain their polarity for up to several hundred femtoseconds-long enough to accelerate charges to very high velocities. An obvious application is thus the study of nonlinearities in the drift velocity of charge carriers and their impact on the system with sub-ps time resolution-something that is not feasible with electrically exerted fields.

GaAs served again to demonstrate this approach ( $\mathrm{Su}$ et al., 2009). Single-cycle $\mathrm{THz}$ pulses with peak fields up to $200 \mathrm{kV} / \mathrm{cm}$ were generated in a large-aperture ZnTe crystal (Blanchard et al., 2007). A single $\mathrm{THz}$ pulse was used to simultaneously perturb and probe carriers, following an optical pump pulse that photoinjected charge carriers in the material. The observed dynamics occurred within the 


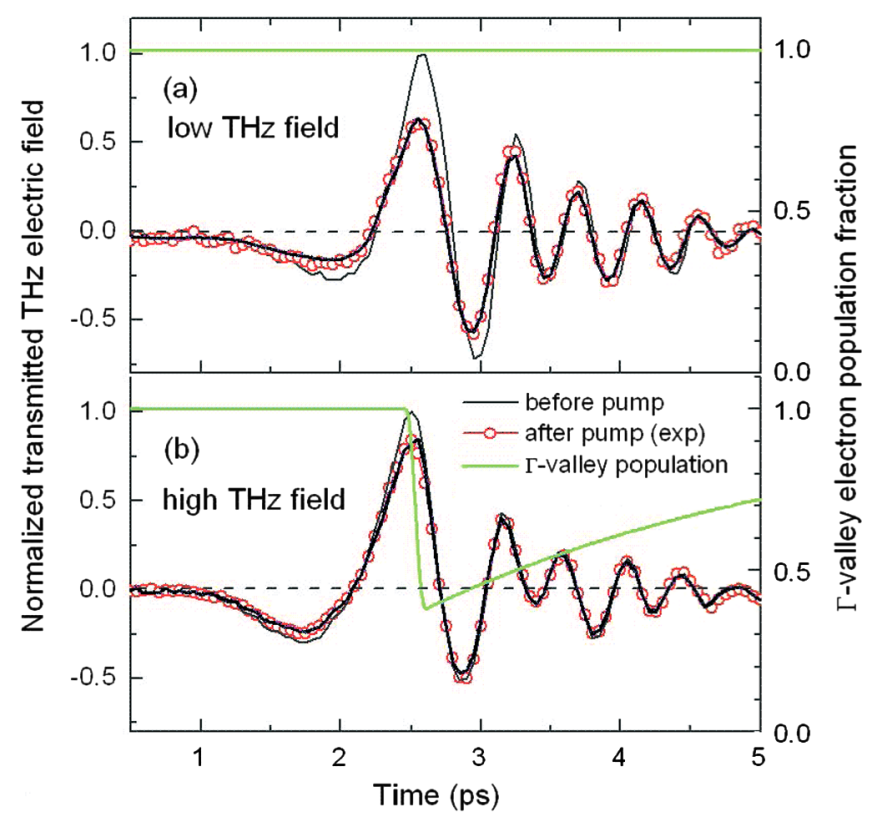

FIG. 11 (color online). Normalized THz electric fields transmitted through a GaAs sample before (thin line) and $10 \mathrm{ps}$ after photoexcitation (open circles) under low (a) and high (b) electric-field strengths. The solid lines represent the calculated fraction of photoexcited electrons in the central $\Gamma$ valley as a function of time. From Su et al. (2009).

bandwidth of the THz-pump pulse or, in other words, on time scales on the order of the pulse duration. Thus, the pump field was modulated as well during propagation. Analysis of the electric-field modulation in the time domain was then applied to infer the underlying processes.

Figure 11 shows the responses with and without optically generated charge carriers for two different $\mathrm{THz}$ field strengths: (a) low field, $4 \mathrm{kV} / \mathrm{cm}$, and (b) high field, $173 \mathrm{kV} / \mathrm{cm}$. Note that the transmission of the highfield beam is significantly enhanced as compared to the low-field case, suggesting a bleaching of the charge carrier absorption at higher fields. Additionally, the trailing part of the waveform features almost no change in amplitude or phase compared to the unexcited case at high $\mathrm{THz}$ fields. This particular behavior originates from the fact that several processes occurring within the pump pulse duration influence the overall conductivity and hence the time-dependent $\mathrm{THz}$ transmission: electrons are accelerated in the electric field to high velocities (which already lowers their mobility and hence bleaches the absorption) until they reach the threshold energy to scatter into the $L$ valley and thus exhibit a lower mobility. This is counterbalanced by backscattering into the $\Gamma$ valley. A model incorporating these and other parameters was implemented to reproduce the time-domain data. The calculated relative $\Gamma$ valley population in time is drawn as a solid line. The time it takes the electrons to be scattered back into the $\Gamma$ valley was calculated to be about 3 ps. This is comparable to the value of $1.9 \mathrm{ps}$ found by $\mathrm{THz}$ pump $\mathrm{THz}$ probe measurements on n-doped GaAs (Hoffmann et al., 2009b). Note that a similar study has dealt with intervalley scattering in n-doped InGaAs thin films using the same experimental setup (Razzari et al., 2009).
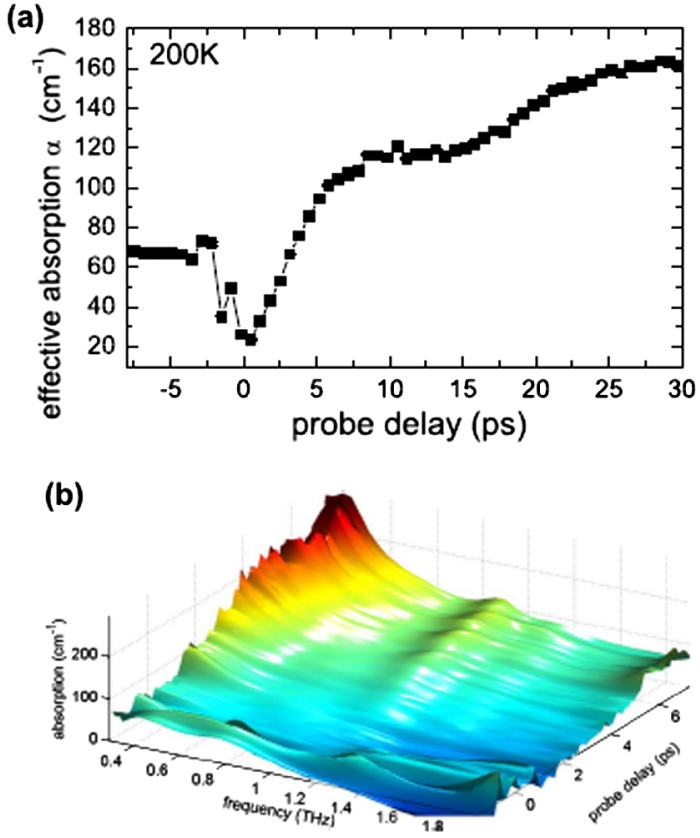

FIG. 12 (color online). THz pump and $\mathrm{THz}$ probe experiment on doped InSb: (a) Spectrally averaged THz absorption as a function of pump-probe delay at a sample temperature of $80 \mathrm{~K}$; (b) frequencydependent absorption coefficient at a sample temperature of $200 \mathrm{~K}$, featuring an increasing Drude part and a rising phonon population after cooling of hot electrons. From Hoffmann et al. (2009b).

Another semiconductor compound on which the effects of high-field low-THz excitation have been studied is InSb (Hoffmann et al., 2009a; Hoffmann et al., 2009b). It possesses one of the highest electron mobility and saturation velocities of all known semiconductors. Even though this enables charge carriers to accelerate to very high velocities in an electric field, intervalley scattering is usually less pronounced - the energetic separation to the next valley of about $1 \mathrm{eV}$ is too large (Chin et al., 1992). However, the band gap of only $170 \mathrm{meV}$ at room temperature is very low. The combination of a high charge carrier saturation velocity and a low band gap facilitates the occurrence of impact ionization: here an electron in the conduction band gains sufficient energy to reach the ionization threshold of an electron in the valence band and excite it into the conduction band. For dc fields this effect can already be observed for field strengths of several hundred $\mathrm{V} / \mathrm{cm}$. The experiments were carried out on $n$-doped $\mathrm{InSb}^{1}$ in a $\mathrm{THz}$ pump-THz probe scheme with the pump reaching field strengths of $100 \mathrm{kV} / \mathrm{cm}$. Both beams were generated by optical rectification in lithium niobate (Hebling, Yeh, Hoffman, Bartal, and Nelson, 2008). Figure 12(a) shows the spectrally averaged dynamics of the absorption for a sample temperature of $200 \mathrm{~K}$. The initial dip in the absorption is due to the common reduction of carrier mobility of hot electrons. Not only does the higher carrier velocity increase the phonon scattering rate, but also the effective mass: strongly nonparabolic bands imply a larger effective mass for higher-energy carriers. Both phenomena

\footnotetext{
${ }^{1}$ As compared to the photoexcited case this implies that only electrons are probed which allows a more direct interpretation.
} 
lower the mobility and thus the absorption. The subsequent absorption rise is caused by both the generation of new carriers through impact ionization and a mobility increase due to carrier cooling. The conductivity evolution shown in Fig. 12(b) reveals another interesting aspect: Apart from the rising Drude part on the low-frequency side a pronounced resonance appears at $1.2 \mathrm{THz}$. This peak was assigned to lattice vibrations: Hot charge carriers in $\mathrm{InSb}$ relax through the emission of optical phonons which in turn decay into acoustic modes. The sum and difference frequencies of these decay channels lie in the $\mathrm{THz}$ range. In particular, the difference frequency between longitudinal optical (LO) and longitudinal acoustic modes at the zone boundary can be found at 1.2 THz. The peak appearance can thus be attributed to a rising phonon population after energy transfer from hot electrons.

b. Dynamics of carrier-lattice coupling It is worth remembering that the collective oscillatory response of a charge carrier plasma to an alternating electric field is governed by many-body correlations: mobile carriers that are displaced from the equilibrium and their associated polarization field exert a restoring force on their surroundings and vice versa. A fundamental question concerns the time scale on which such many-body correlations form after charge carriers are injected in the conduction band-whether it is, for instance, instantaneous.

Huber et al. (2001) addressed this issue in one of the first publications that took advantage of the high time resolution and large bandwidth of mid-infrared (MIR) probes generated by 10 fs oscillator pulses in GaSe. The material of choice was GaAs. In a later publication by the same group the measurements were extended to InP, a semiconductor with a higher Fröhlich constant compared to GaAs (Huber, Kubler et al., 2005). The dynamics showed a similar evolution as in GaAs but spectral signatures that are characteristic for coupling between plasmon and phonon were more pronounced.

Figure 13(a) shows the temporal buildup of the plasma correlation in InP for increasing pump-probe delays. One should note that longitudinal modes of oscillation, such as bulk plasmons and longitudinal optical phonons, manifest themselves as nodes in the dielectric function (Kittel, 1995; Hasselbeck et al., 2002). For this reason, the inverse dielectric function, also known as the dielectric loss function $1 / \epsilon$, is plotted. For the unexcited material, the dielectric loss function features only one peak due to the longitudinal optical phonon. This resonance broadens and shifts to higher frequencies once electron-hole pairs have been injected. Within about $100 \mathrm{fs}$, the bandwidth narrows and eventually a peak appears at the plasma frequency of $14.4 \mathrm{THz}$. This behavior highlights the evolution from an uncorrelated electron-hole gas to a collective plasmon excitation that is mediated by many-body correlations, as shown in Fig. 13(b). The buildup time decreases with the charge carrier density; it scales with the inverse of the plasma frequency [see Fig. 13(c)]. The emergence of the bulk plasmon peak is accompanied by the appearance of a second smaller peak below the transverse optical phonon. This behavior is a direct consequence of the coupling between plasmon and phonon that forms a mixed mode. It causes a splitting of the normally linear plasmon dispersion into two branches: the upper mode $L_{+}$and the
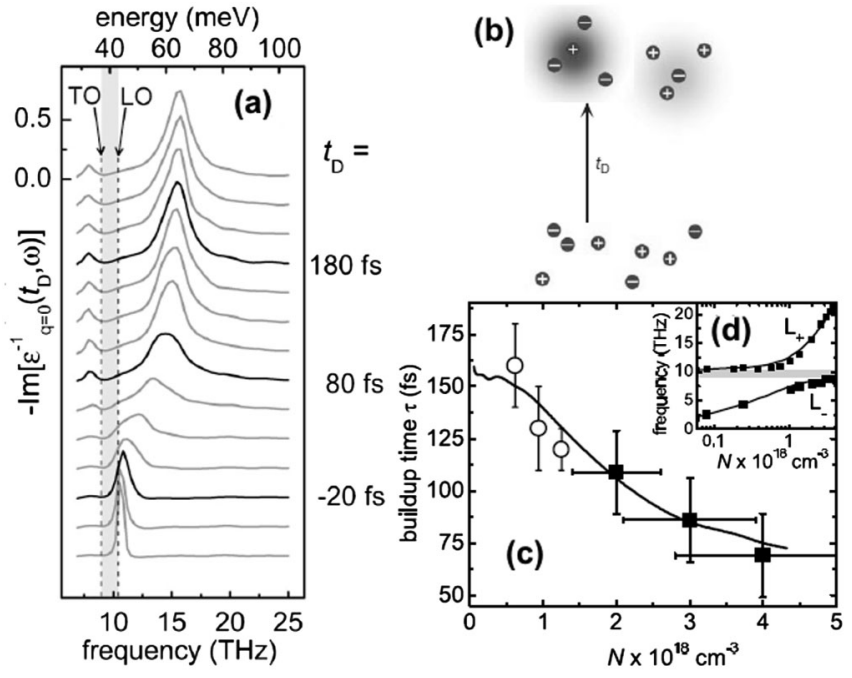

FIG. 13. (a) Imaginary part of the inverse dielectric function of InP vs frequency for various pump-probe delays. The grey region indicates the Reststrahlenband; (b) visualization of the transition of bare charges to a correlated screened charge carrier plasma; (c) plot of the buildup time $\tau$ for plasmon-phonon coupling vs excitation density; (d) spectral positions of the $L_{+}$and $L_{-}$modes for various excitation densities. From Huber, Kubler et al. (2005).

lower mode $L_{-}$. Mapping the resonance frequencies as a function of charge density sketches the dispersion of the two branches [Fig. 13(d)]. The disappearance of the bare phonon resonance that is visible before carrier injection provides another indication for the existence of a mixed mode: its spectral weight shifts into the coupled modes once the plasmon-phonon coupling has been established.

The question of how the internal motion of a polaron within its potential well is affected at high electric fields was recently discussed in an experiment on GaAs by Gaal et al. (2007). To illustrate the physical mechanisms, the selfinduced potential for a single polaron was calculated and is shown in Fig. 14 for low (a) and high (b) electric fields. A weak electric field merely induces a drift motion of the polaron (a). The electron stays at the center of the potential and the polaron response is determined by the center-of-mass motion of the entire quasiparticle. At high electric fields the electron gets appreciably displaced from the potential minimum [Fig. 14(b), filled circle). When the polaron reaches the saturation velocity, i.e., its energy equals the optical phonon energy, it impulsively transfers its energy to the lattice by emitting an optical phonon. The coherent oscillations of the emitted phonon appear as a stern wave of the moving electron. At a certain threshold, the induced oscillatory polarization changes are strong enough to modulate the lattice potential and alter the electron motion. The electron now oscillates within the potential with the optical phonon frequency [Fig. 14(b), open circles) on top of the polaron drift motion. The experiment succeeded in probing this wiggling motion by detecting the radiation emitted from the moving charge.

In the actual experiment a low- $\mathrm{THz}$ beam with a field strength of $20 \mathrm{kV} / \mathrm{cm}$ provided the electric field [Fig. 14(c) top) and a MIR beam with a center frequency of $17 \mathrm{THz}$ served as the probe. The combination of both beams 


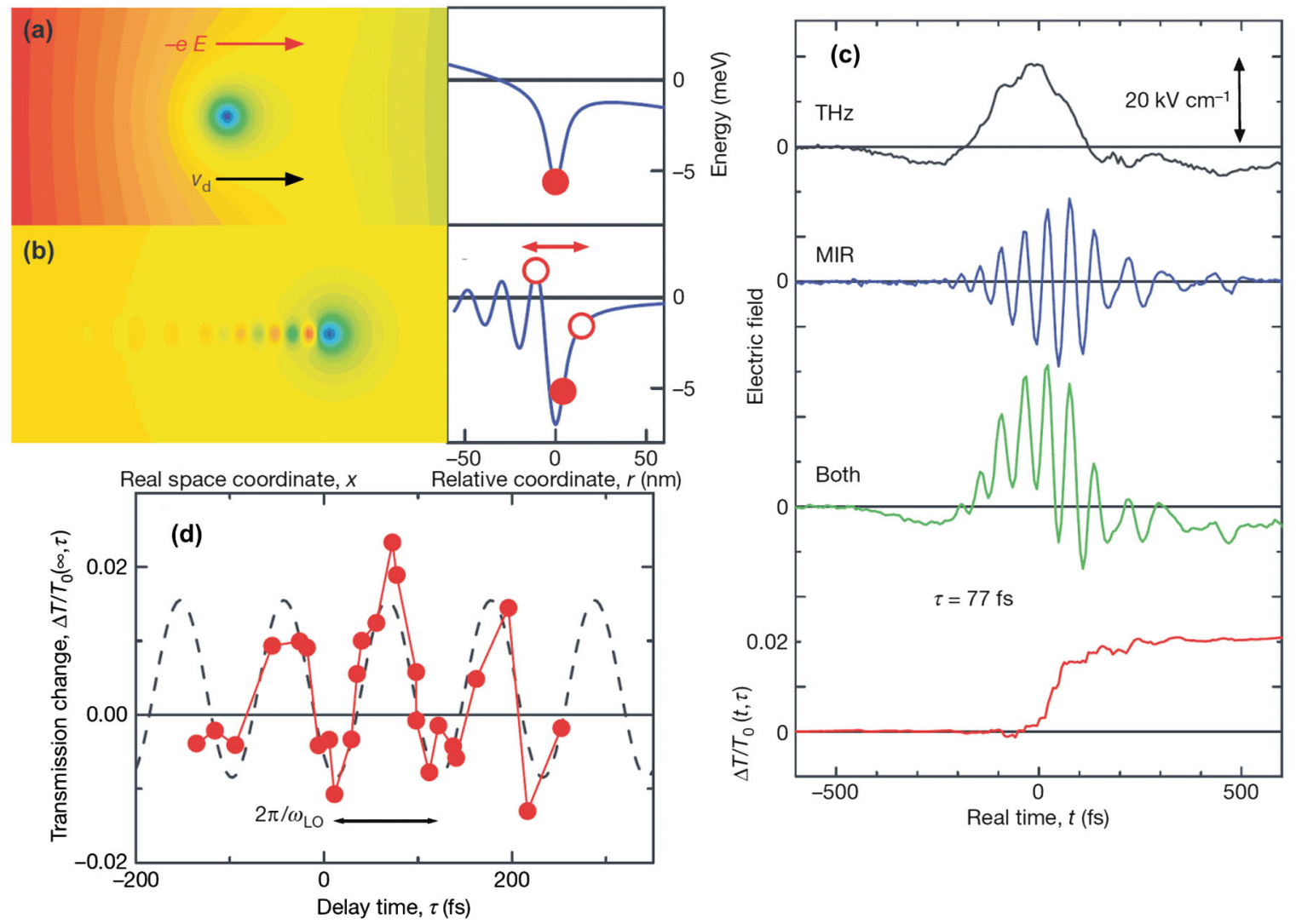

FIG. 14 (color online). Self-induced polaron potential of a polaron at (a) weak and (b) strong electric fields. Left side: contour plot of the polaron potential. Right side: polaron potential in coordinates relative to the quasiequilibrium position of the electron (dot); the circles illustrate the electron oscillation within the polaron potential at high electric fields. (c) Measured transients for a pump-probe delay of 77 fs; the bottom graph shows the time-integrated transmission change $\Delta T / T$; (d) transmission change $\Delta T / T$ as a function of pump-probe delay $\tau$. From Gaal et al. (2007).

for a certain pump-probe delay $\tau$ is also shown. The time-integrated transmission should normally be zero, which is the case for low electric fields. For fields $>10 \mathrm{kV} / \mathrm{cm}$ however a significant change in transmission occurs, as indicated for a pump-probe delay of 77 fs. Figure 14(d) shows that the magnitude and sign of the transmission change depends on the pump-probe delay. More precisely, it is modulated with a period equaling the LO phonon period (indicated as a dashed line). Apparently, the probe beam transmission is influenced by the radiation emitted from the wiggling charge, modulating it between absorption and gain.

In this study the MIR beam was used as a probe that coherently mixes with radiation emitted from the system and thus gets modulated-similar to well-known coherent wave mixing techniques in the visible or infrared region. This probe scheme is thus somewhat different from the cases treated before in which the probe essentially interacts with an incoherent entity like an electron-hole gas. This wave mixing approach has been used in many cases to study coherent polarization dynamics in semiconductors (Chemla and Shah, 2001; Axt and Kuhn, 2004).

It seems reasonable to address the influence of chargelattice interactions in organic semiconductors as well. Compared to their inorganic counterparts they usually possess a multitude of vibrational modes, both intramolecular and intermolecular. And as it turns out, charge carriers in organic semiconductors can be of very polaronic nature and polaron binding energies ${ }^{2}$ of $100 \mathrm{meV}$ or more are not uncommon. This strong coupling suggests that injected charge carriers significantly alter the potential landscape of their host molecules-something that should be visible spectroscopically.

Koeberg et al. (2007) investigated the ultrafast response of intramolecular vibrations after photoexcitation in rubrene, a small-molecule organic semiconductor that possesses one of the largest mobility values. The static absorption and dispersion spectrum is shown in Fig. 15(a). The featured resonances are intramolecular vibrations; whereas, intermolecular modes are restricted to lower frequencies $(<6 \mathrm{THz})$. The photomodulated spectrum for different pump-probe delays in the first picosecond is shown in Fig. 15(b). Analysis shows that it contains photoinduced modulations of the vibrational resonances carried on a nonresonant background absorption that originates from the spectrally broad free-carrier response. A close-up of the resonance around $15.5 \mathrm{THz}$ in Fig. 15(c)

\footnotetext{
${ }^{2}$ Having its roots in the field of chemistry rather than solid-state physics the polaron binding energy in organic semiconductors is often referred to as reorganization energy instead, which originates from electron transfer theory. We refer to Coropceanu et al. (2007) for more theoretical aspects of charge transport in organic semiconductors.
} 

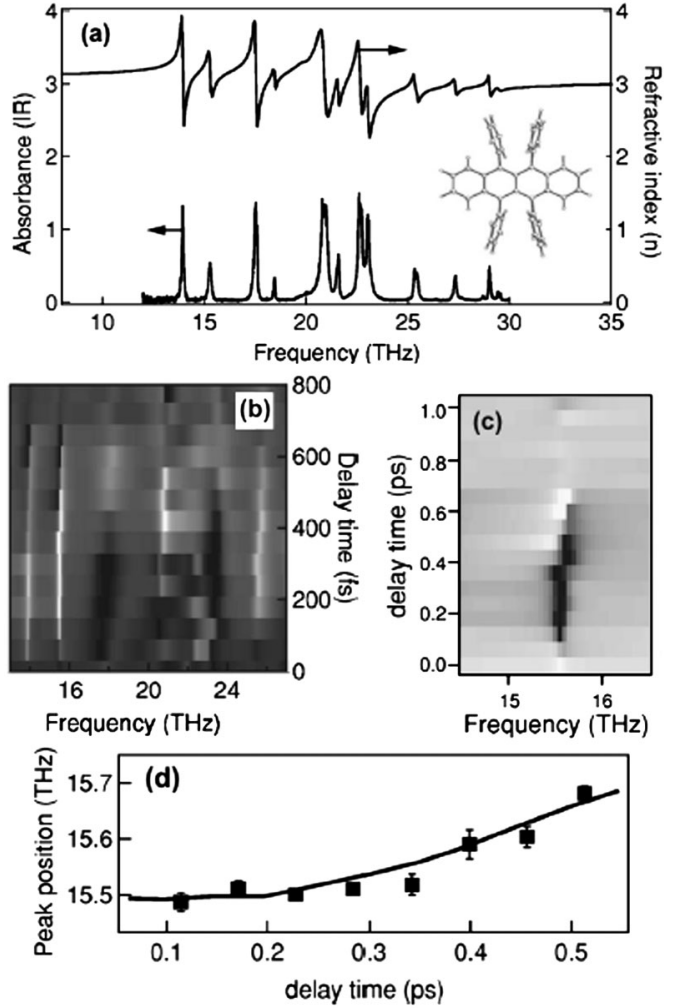

FIG. 15. (a) Linear absorption spectrum and refractive index of a rubrene crystal; the inset shows its molecular structure; (b) frequency-resolved real part of the transmsission change $\Delta E / E$ as a function of pump-probe delay; (c) close-up of the transmission change of the resonance around $15.5 \mathrm{THz}$; (d) experimental (squares) and modeled (solid line) shift of the resonance frequency vs pump-probe delay. From Koeberg et al. (2007).

reveals more details: The photomodulation of the mode lasts merely for 700 fs and its resonance frequency increasingly shifts in time to the blue [Fig. 15(d)]. The mechanisms responsible for this behavior are basically related to charge injection and redistribution which in turn alters the vibrational landscape. Both direct excitation of the vibronic states of the rubrene chromophores and their subsequent relaxation via internal conversion affects the vibrational potentials and frequencies. The magnitude of the observed resonance shift can thus be seen as a measure for the electron-phonon coupling strength.

\section{B. Excitons in bulk materials}

Electrons and holes created after photoexcitation across the band gap are not necessarily fully decoupled from each other. The attractive Coulomb force between them may lead to the formation of excitons, i.e., bound electron-hole pairs, particularly at low temperatures and for low-dielectric materials, in which screening of the charges is relatively inefficient. The binding energy associated with excitons lies generally in the 1-100 meV range, and intraexcitonic transitions are typically a fraction of the binding energy (Kira et al., 2001; Klingshirn, 2006; Koch et al., 2006).

The existence of excitons in a semiconductor has important implications on its electric properties. While an electric field acting on free charge carriers is capable of inducing a preferential drift and hence can produce an electric current, bound electron-hole pairs are merely displaced and no net force can be exerted. In a solar cell, for instance, this implies that photogenerated electron-hole pairs that are excitonic in nature are effectively bound and cannot contribute to the photocurrent. The identification and characterization of excitonic populations is therefore of practical importance.

Excitons may have signatures in the optical spectrum as sharp lines that are redshifted from the electronic band gap by their binding energy. Photoluminescence and absorption measurements of these peaks have traditionally provided the means to identify excitons. Their quantitative and unambiguous assessment is, however, not straightforward. Because of momentum conservation luminescence can only be observed from excitons with a total momentum close to zero, especially in the case of small exciton-phonon coupling. So-called "dark" excitons with a forbidden dipole moment for interband transitions are not accessible with visible or near-IR probes either. An additional complication may be noted from recent calculations and experiments, which have shown that emission from the alleged exciton resonance can originate from an unbound electron-hole plasma as well (Kira et al., 1998; Galbraith et al., 2005).

A direct way to monitor excitons that does not rely on probing interband transitions is therefore desirable. $\mathrm{THz}$ spectroscopy can detect excitons, both through resonant interactions with internal exciton transitions and through a nonresonant interaction, i.e., through the polarizability associated with the electron and hole wave functions of the exciton.

\section{Intraexcitonic transitions}

Many of the following experiments were performed on stacked GaAs quantum well samples rather than bulk materials and hence on two-dimensional electron-hole gases. There are several reasons for that: Epitaxially grown, these layers are virtually strain-free and of high purity. Quantum confinement effects increase band gap and the exciton binding energy of originally $4.2 \mathrm{meV}$ and can be tuned by changing the well thickness. The degeneracy of the valence band at $k=0$ is lifted and hence heavy and light hole levels are split. The band gap can be tuned to $1.55 \mathrm{eV}$ to match the output energy of Ti:sapphire lasers which means that resonant exciton or continuum excitation is possible without the use of optical frequency conversion processes. Furthermore, since the quantum well (QW) thickness of around $10 \mathrm{~nm}$ is much smaller than the pump and probe wavelengths in use, propagation effects in the medium can be neglected. The concepts inferred from the studies of these samples can nevertheless be transferred to the three-dimensional bulk case.

The first detailed study on the dynamics of the formation and ionization of excitons was performed by Kaindl et al. (2003) on GaAs quantum wells. Later works by this group incorporated a more elaborate analysis in order to retrieve precise quantitative figures on exciton and plasma densities (Kaindl et al., 2009). The quantum wells had a thickness of $14 \mathrm{~nm}$ which led to a $1 s-2 p$ transition energy of $7 \mathrm{meV}$ $(\sim 2 \mathrm{THz})$ — and this "exciton fingerprint" being perfectly accessible by the probe $\mathrm{THz}$ spectrum that spanned from 
2 to $12 \mathrm{meV}$. The optical pump spectrum centered around $800 \mathrm{~nm}$ was spectrally narrowed to about $1 \mathrm{meV}$ in order to selectively excite into either exciton or continuum states. Two scenarios were investigated:

(1) To observe condensation of an unbound plasma into excitons, the sample was kept at a temperature of $6 \mathrm{~K}$ and free charge carriers were created by excitation into the continuum.

(2) Exciton ionization dynamics were measured after resonant excitation of the 1s heavy-hole transition, with the sample held at temperatures ranging from 10 to $80 \mathrm{~K}$.

Regarding (1), directly after continuum excitation the spectrum is as expected dominated by the Drude response of free carriers. Surprisingly, however, already $40 \%$ of the electron-hole pairs are bound quasi-instantaneously (within the experimental time resolution of about $1 \mathrm{ps}$ ), indicated by a pronounced absorption peak at $7 \mathrm{meV}$ [Fig. 16(a)]. Timeresolved photoluminescence measurements show no emission at these early time delays which led to the conclusion that these excitons occupy high-energy states with momenta $k>0$ and, as a result, are dark. This fast initial generation of excitons is followed by a slower condensation process on $\sim 100$ ps time scales that leads to $90 \%$ of the electrons and holes being converted into excitons at $1 \mathrm{~ns}$ after photoexcitation. This process is accompanied by a rise of the exciton luminescence occurring on comparable time scales. Relaxation during the slow part involves at first the emission of optical and subsequently of acoustic phonons. The origin for the quasi-instantaneous exciton formation is as yet not understood in detail. Condensation facilitated by intricate energy and momentum exchange events between carriers, rather than a phonon bath, provides an explanation that is consistent with the observed time scale.

Regarding (2), resonant interband excitation into the heavy-hole state initially creates a coherent interband polarization that dephases via phonon and carrier scattering within 5 ps into an incoherent exciton population (Lovenich et al., 2002). Accordingly, the anticipated $\mathrm{THz}$ exciton signature
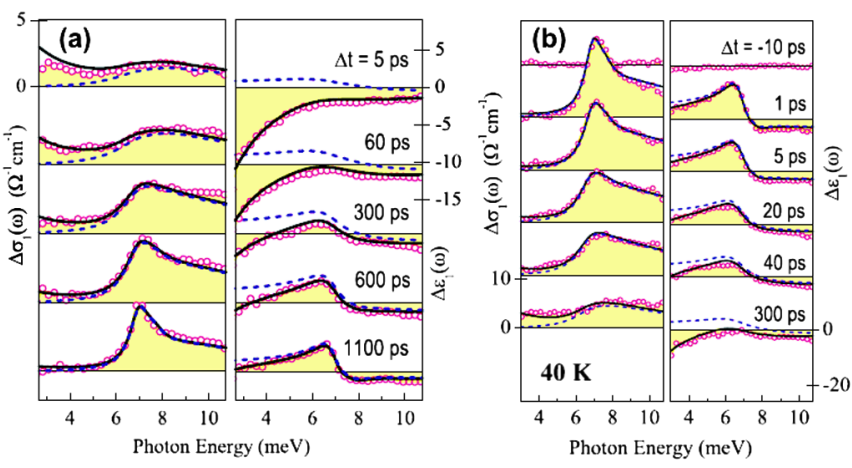

FIG. 16 (color online). Transient terahertz spectra of charge carriers in GaAs quantum wells at different pump-probe delays: (a) Nonresonant photoexcitation into the continuum at a sample temperature of $6 \mathrm{~K}$ shows the formation of excitons from an unbound charge carrier plasma; (b) resonant generation of exciton states at a sample temperature of $40 \mathrm{~K}$ and subsequent ionization of excitons. The respective left sides of the plots show the real part of the conductivity, the right side the real part of the dielectric constant of the charge carrier gas. From Kaindl et al. (2009). appears. At a sample temperature of $10 \mathrm{~K}$ the exciton fraction stays practically constant for all time delays, indicating that the thermal energy of the lattice is not sufficiently high to ionize a significant fraction of excitons. At higher temperatures, excitons get ionized on a time scale of several hundred picoseconds until they reach their temperature-dependent quasiequilibrium exciton concentration. As an example, the $\mathrm{THz}$ spectra for different pump-probe delays at $T=40 \mathrm{~K}$ are plotted in Fig. 16(b). At that temperature, the charge carrier plasma reaches its final exciton fraction of $60 \%$ after 400 ps. Above $50 \mathrm{~K}$ the ionization rate increases rapidly since now the ionization is facilitated through the absorption of LO phonons. At $80 \mathrm{~K}$ the quasiequilibrium exciton fraction of $30 \%$ is already established after 30 ps.

The mentioned experiments were performed at relatively low charge carrier densities of around $10^{10} \mathrm{~cm}^{-2}$. An interesting change in the $\mathrm{THz}$ response occurs with increasing excitation fluence (Huber, Kaindl et al., 2005). For a GaAs QW sample held at a temperature of $6 \mathrm{~K}$, optically exciting at the exciton line results in the formation of excitons of densities up to $5 \times 10^{10} \mathrm{~cm}^{-2}$. When the excitation fluence results in densities exceeding this number, however, absorption starts to build up at low frequencies, indicative for the appearance of a conducting phase. The spectrum starts to deviate from being purely excitonic and the addition of a Drude component is required in order to describe it. Higher charge densities amplify this effect until beyond a critical density of $2 \times 10^{11} \mathrm{~cm}^{-2}$, where all exciton signatures have vanished and the spectrum can be fully accounted for by the Drude model. This so-called excitonic Mott transition from an insulating phase at low charge carrier densities to a conducting one at high densities is driven by the increasing influence of Coulomb interactions between carriers. These many-body effects are usually negligible in a dilute electronhole gas but become relevant at high charge densities when interexciton distances become comparable to the exciton Bohr radius. In that case, the electric field exerted by adjacent charge carriers is increasingly screening the attractive force between electrons and holes, thereby lowering the binding energy of excitons.

This lowering of the exciton binding energy can also be observed directly in the experiment: In the low-density case, in which the fraction of bound and unbound species in (quasi) equilibrium is essentially governed by the sample temperature, the $\mathrm{THz}$ spectrum simply reflects the relative contributions from excitons and plasma, but these individual contributions remain unchanged. During the Mott transition, however, the excitonic fine structure as seen in the $\mathrm{THz}$ spectrum is modified as well, reflecting the density-dependent influence of many-body correlations: The $1 s-2 p$ transition energy decreases with increasing carrier density. This is a direct consequence of a lower exciton binding energy. This effect can also be observed in luminescence and absorption measurements as a blueshift of the exciton line towards the electronic band gap. Moreover, the $1 s-2 p$ linewidth increases with charge density. The dephasing time of the polarization induced between the $1 s$ and $2 p$ states is reduced due to the growing perturbation from adjacent charge carriers.

Similar observations have been made in a study on the bulk material zincoxide $(\mathrm{ZnO})$, where at low temperatures 
excitons are readily formed (Hendry, Koeberg, and Bonn, 2007). The exciton binding energy of $\mathrm{ZnO}$ is $60 \mathrm{meV}$ and hence several times higher than GaAs. The first exciton transition lies at $8 \mathrm{THz}$ and can thus not be detected resonantly in the ZnTe probe window. However, its existence can be sensed off-resonantly by tracing its influence on the phase of the transmitted $\mathrm{THz}$ pulse, i.e., on the imaginary part of the conductivity (see Fig. 4). At low excitation densities, where the average exciton-exciton distance is much larger than the Bohr radius in $\mathrm{ZnO}$, initially free charges are formed, as evidenced by the Drude-like behavior of the conductivity at short times after excitation. After $\sim 50 \mathrm{ps,} \mathrm{the} \mathrm{imaginary}$ component of the conductivity switches sign: from positive to negative. An analysis of the terahertz spectra reveals that this sign change originates from the conversion of free charges into excitons. This sign change can be understood by noting that the "resonance frequency" shifts from zero (Drude response) to finite frequency (exciton resonances). Indeed, at sufficiently long times the terahertz response can be described completely by the exciton response. For $\mathrm{ZnO}$, at low temperatures the majority of the initially excited electron-hole pairs forms excitons.

The exciton formation dynamics can be characterized by an exponential decay time of $20 \mathrm{ps}$ which is associated with the disappearance of the Drude response. This relatively slow evolution of conducting plasma into an exciton gas was explained by acoustic phonon emission being the rate determining step for exciton formation. This scenario contradicts the hypothesis that excitons are formed in the excited state, whereupon exciton cooling takes place ("hot exciton cascade"). In the hot exciton cascade picture, rapid emission of optical phonons by photocarriers leads to the formation of hot excitons, which subsequently cool to the emissive $(K=0)$ state by slow acoustic phonon emission [Fig. 17(a)].
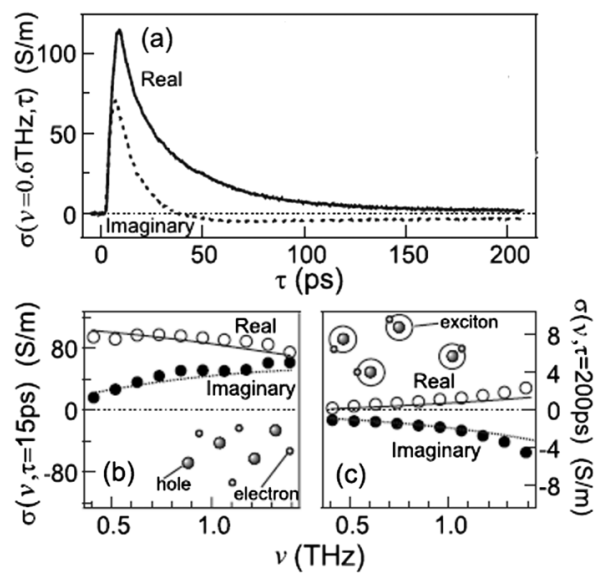

FIG. 17. (a) Evolution of the real (solid line) and imaginary (dashed line) conductivity after photoexcitation of low charge carrier densities in $\mathrm{ZnO}$ at $30 \mathrm{~K}$; (b) complex conductivity $15 \mathrm{ps}$ after photoexcitation showing the Drude response of free carriers (solid and dashed lines are real and imaginary conductivity following the Drude model); (c) complex conductivity 200 ps after photoexcitation showing the off-resonant response of intraexcitonic transitions (solid and dashed lines are real and imaginary conductivity of an excitonic response). From Hendry, Koeberg, and Bonn (2007).
However, emission of optical phonons by photocarriers in semiconductors typically occurs on subpicosecond time scales and one would expect disappearance of the Drude response on this time scale if the hot exciton cascade hypothesis were correct.

The formation of "cold" excitons from carriers, with acoustic phonon emission being the rate-limiting step, is corroborated by the temperature dependence of exciton formation. The temperature dependence of the exciton formation process is in good agreement with the temperature dependence expected for acoustic phonon scattering; the much stronger dependence expected for longitudinal optical phonons (expected in the hot exciton cascade picture) is not borne out experimentally.

At significantly higher excitation densities, where the interexciton distance is appreciably less than the Bohr radius, the photoconductivity reflects that of a Drude system with very high scattering rates. These high scattering rates can be attributed to carrier-carrier interactions. For these high densities, the electron-hole gas does not decay into an exciton state, but rather into an electron-hole plasma characterized by a density corresponding to an average carrier-carrier distance of roughly the Bohr radius. Apparently, above the Mott density, the initial high-density electron-hole plasma decays very rapidly (1.5 ps) through Auger annihilation to reach this value. In contrast to exciton formation, annihilation is found to be independent of lattice temperature, occurring while the plasma is still hot.

There has been some debate whether the description of the results in both the quantum wells and bulk materials in terms of a simplified two-component picture as a sum of a Drude and exciton transition is appropriate. Kaindl et al. (2009) validated this approach by calculating the exciton fractions for both excitation scenarios at different time delays using the Saha equation. Derived from basic thermodynamic relations, this equation assumes that excitons and free carriers reach a quasiequilibrium and their according densities can be deduced solely from the carrier temperature. Seasoned with literature values for the electron-phonon coupling strength the cooling dynamics can be calculated-which turned out to be consistent with the measured $\mathrm{THz}$ spectra.

On the other hand, it was shown recently that Coulomb correlations between electrons or holes are always present in the system, which can therefore not be completely adequately described as being composed of simply a mixture of an exciton gas and a free electron-hole plasma. A microscopic theory has suggested that one in fact has to distinguish three, rather than two, components: a continuum of ionized excitons, intermediate exciton states, and a Coulomb-correlated electron-hole plasma. It was shown that if this is not done correctly, it is difficult to quantitatively interpret the results in terms of relative contributions from the excitons and the electron-hole plasma (Kira and Koch, 2006; Koch et al., 2006).

Below a certain critical temperature an electron-hole gas can undergo a phase transition into an electron-hole liquid in the form of small droplets (EHD) that coexist with the gas phase of free carriers and excitons. The formation of a liquidlike phase is basically restricted to indirect semiconductors since the carrier lifetime is sufficiently long to reach the 
required charge carrier temperature and to spatially separate liquid and gas phases. In analogy to a real liquid the electronhole liquid density only depends on its temperature and not the pump power ("pressure"). Instead, higher pump powers merely increase the volume ratio between liquid and gas fractions. The majority of studies on the properties of EHDs in indirect semiconductors such as Si and Ge originate from the 1970s and early 1980s (Jeffries, 1975; Shah et al., 1977). Because of technical limitations, these measurements were limited to the steady state; only recently have ultrafast spectroscopy methods permitted the time-resolved observation of EHD formation, most notably through photoluminescence measurements.

For silicon, the temporal buildup of excitons and electronhole droplets after photoexcitation has recently been measured using THz TDS (Suzuki and Shimano, 2009). A pair of $\mathrm{GaP}$ crystals were used for terahertz generation and detection which provided a probe spectrum ranging from 0.5 to $6 \mathrm{THz}$ (2-25 meV). The $e-h$ phase diagram for silicon is shown in Fig. 18(a). The two dashed lines represent different calculations for the Mott density, i.e., the critical carrier density for the metal-insulator transition. Measurements were performed at charge carrier densities of $1 \times 10^{16} \mathrm{~cm}^{-3}$ and $1 \times$ $10^{17} \mathrm{~cm}^{-3}$, i.e., below and above the Mott density, respectively. A sample temperature of $5 \mathrm{~K}$ assured that for both excitation scenarios the $e-h$ system would reside in the two-phase region, i.e., an initial $e-h$ plasma is expected to condense into excitons and droplets.

The probe spectra for the high-density case at different pump-probe delays are shown in Fig. 18(b). At early times the response can roughly be described by the Drude model but excitonic signatures, recognizable by the appearance of the $1 s$ - $2 p$ transition at about $11 \mathrm{meV}$, are already visible. Within 400 ps the free-carrier response vanishes, accompanied by an increase of the exciton resonance and a broad high-frequency absorption. The exciton resonance subsequently decays and has all but disappeared after $2 \mathrm{~ns}$ whereas the magnitude of the broad high-frequency absorption stays practically constant. They assigned this broad absorption peak to $e-h$ droplets and suggested the following scenario: after photoexcitation, free carriers simultaneously condense within 500 ps into excitons and EHDs. Subsequently, excitons diffuse into EHDs where they are efficiently screened by the $e-h$ liquid and are hence not visible to the THz probe. After 2 ns, practically only EHDs are left in the system.

The EHD resonance can be understood by considering the droplet as being a conducting sphere surrounded by a dielectric (excitonic) medium. It is hence analogous to the observation of a plasmonic response of free carriers in nanoparticles (see the next section). They thus modeled the resonance using the Bruggemann formula as an effective medium description (see Sec. II.D.2). Measurements at low charge carrier densities of $1 \times 10^{16} \mathrm{~cm}^{-3}$ (not shown), i.e., below the Mott transition, yielded similar exciton condensation time scales of a few hundred picoseconds, with the EHD resonance, however, being much less pronounced and only visible as a slight side shoulder next to the exciton resonance.

The conversion of free carriers into excitons has also been observed in polymeric semiconducting materials. Semiconducting conjugated polymers have received considerable interest owing to their potential in technological applications, particularly in electronics. Despite their widespread optical applications, the nature of the photoexcitation physics in these materials has been subject to intense debate. One of the key questions that remained controversial was whether, initially upon photoexcitation, excitons or charge carriers are primarily formed. THz TDS was ideally suited to resolve this controversy, with its ability to monitor the evolution of free and bound charges on subpicosecond time scales following photoexcitation, through the time-dependent real and imaginary components of frequency-dependent conductivity. This allowed for an unambiguous assignment of the
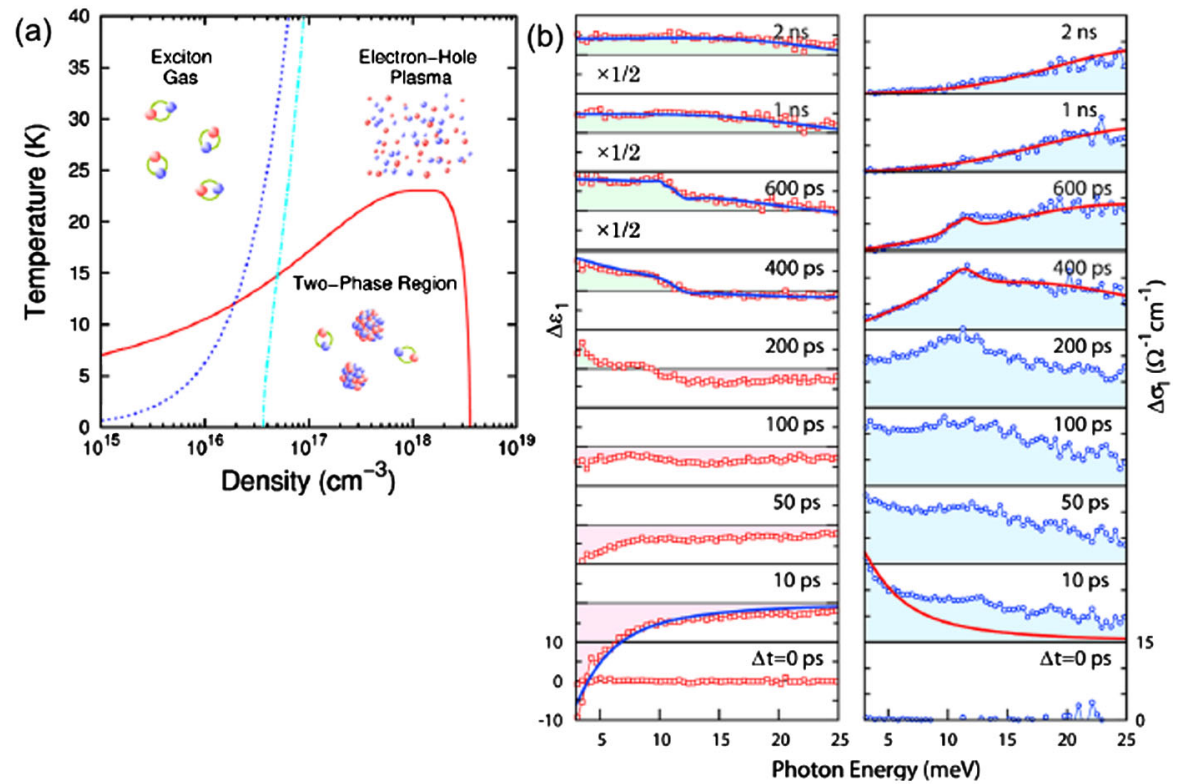

FIG. 18 (color online). (a) Phase diagram of an electron-hole system in silicon; (b) real part of the dielectric function (left) and conductivity (right) of the photoexcited plasma at high excitation densities for various pump-probe delays. From Suzuki and Shimano (2009). 
excited species during the excitation process. For the semiconducting polymer poly(2-methoxy-5-(2'-ethyl-hexyloxy)p-phenylene vinylene) (MEH-PPV), it was found that only a very small fraction $\left(<10^{-2}\right)$ of photons resulted in direct excitation of free-electron-hole pairs, whereas the majority of the excitation resulted in exciton formation (Hendry, Schins et al., 2004). Free charges are generated from the dissociation of nascent, hot excitons, and are extremely short lived $(\sim 1 \mathrm{ps})$.

Akin to works on intersubband transitions in quantum wells, which are covered in Sec. IV.B.1, THz measurements on intraexcitonic transitions have recently provided a new playing field to observe quantum phenomena such as stimulated emission, quantum beats (Huber et al., 2006), and Rabi cycling (Leinss et al., 2008). The key advantage here lies in its electro-optic detection scheme which permits the phaseresolved probing of pump-induced polarizations with a time resolution in the subcycle regime. This feature has so far been hard or impossible to achieve in the infrared or visible. In the following, we focus on the observation of Rabi cycles between intraexcitonic transitions in $\mathrm{CuO}_{2}$ by Leinss et al. (2008) since it provides another example of how high-field terahertz pulses can drive optical transitions in the nonlinear regime.

Excitons in $\mathrm{CuO}_{2}$ possess a particularly large binding energy of $150 \mathrm{meV}$. Strong exchange interaction splits their $1 s$ ground state by $12 \mathrm{meV}$ into an ortho and a lower-lying para variety [see Fig. 19(a)] (O'Hara and Wolfe, 2000;

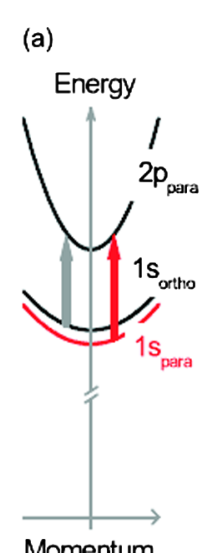

Momentum
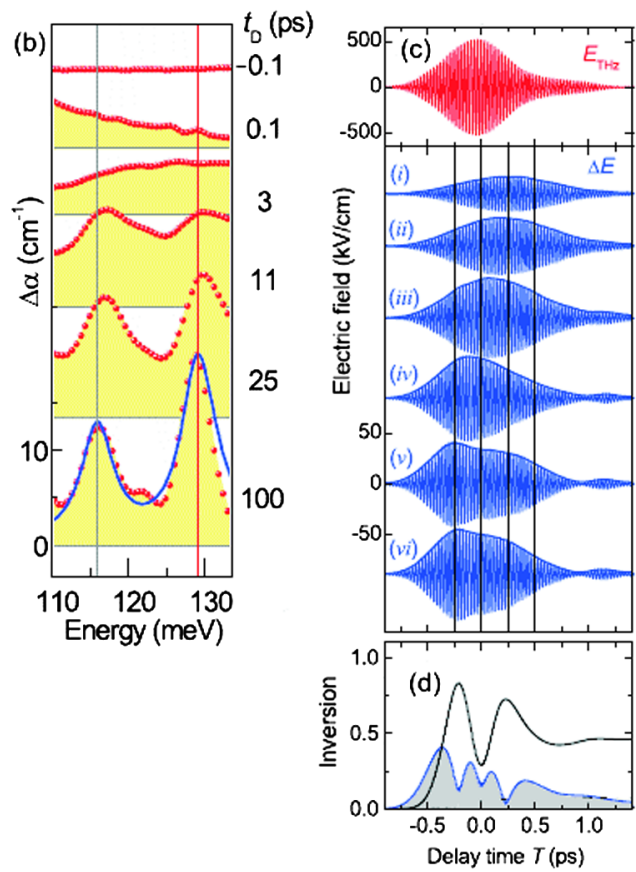

FIG. 19 (color online). (a) Sketch of the first intraexcitonic transitions of $\mathrm{CuO}_{2}$ orthoexcitons and paraexcitons in momentum space; (b) pump-induced absorption change $\Delta \alpha$ for various pump-probe delays featuring the $1 \mathrm{~s}-2 \mathrm{p}$ transitions of ortho and para excitons; (c) upper panel: profile of the exciting $\mathrm{THz}$ pulse; lower panel: Reemitted THz fields for driving field strengths reaching from $0.07 \mathrm{MV} / \mathrm{cm}$ (i) to $0.5 \mathrm{MV} / \mathrm{cm}$ (vi); (d) calculated inversion (solid line) and polarizability (shaded area) of the paraexciton $1 s-2 p$ two-level system. From Leinss et al. (2008).
Kubouchi et al., 2005). After pumping with $1.55 \mathrm{eV}$ charge carriers are generated via two-photon excitation that show a free-carrier response at early times [see Fig. 19(b)]. Within $100 \mathrm{ps}$, these have largely condensed into excitons, as evidenced by the appearance of the $1 s-2 p$ transitions of ortho $(116 \mathrm{meV})$ and para $(129 \mathrm{meV})$ excitons. In order to resonantly excite the $1 s-2 p$ para transition, pulses with a photon energy of $129 \mathrm{meV}$ and a width of $4 \mathrm{meV}$ were generated by optical rectification of $0.2 \mathrm{~mJ}$ in a GaSe crystal. In this way, peak fields of up to $0.5 \mathrm{MV} / \mathrm{cm}$ could be generated in the focal spot [see Fig. 19(c) upper panel).

The response for six different peak fields are shown in Fig. 19(c), (i)-(vi). For low excitation fields [traces (i) and (ii)], the reemitted field reaches its maximum towards the end of the driving pulse and dephases within 0.7 ps. At higher excitation intensities [traces (iii)-(vi)]. however, the response does not simply scale in magnitude. Instead, the reemitted field increasingly rises more rapidly and its peak shifts to earlier times. For the highest intensities, it reaches its maximum before the peak of the pump pulse. At the same time, the peak amplitude saturates and a second less pronounced peak appears about $0.5 \mathrm{ps}$ after the first peak. Apparently, the driving field saturates the $1 s-2 p$ transition and induces a coherent nonlinearity that leads to an oscillatory behavior.

The authors analyzed the results employing a microscopic description of the intraexcitonic light-matter coupling [see Kira and Koch $(2004,2006)]$. This involved the correction of the response for the ponderomotive current originating from existing free carriers and ionized excitons. The calculated dynamics of the $1 s-2 p$ transition are plotted in Fig. 19(d). The population inversion (solid line) and the polarizability (shaded area) feature approximately two Rabi cycle periods and the inversion reaches peak values of $80 \%$.

\section{NANOSTRUCTURED SEMICONDUCTORS}

The ability to characterize electrical properties in a noncontact fashion with subpicosecond temporal resolution is necessary in the field of nanoscale electronics and optoelectronics, where it is very challenging, if not impossible, to use conventional probes. In this section, we distinguish two types of nanostructured semiconductors: those where electrons and holes are present as free charge carriers, albeit impeded in their movement, and those where quantum confinement effects dominate the response of the carriers. The former class of materials are relatively large semiconductor structures, or sintered oxide particles. The latter includes quantum wells, quantum dots, and graphitic nanostructures.

\section{A. Structures with no quantum confinement}

\section{Carrier localization}

Pure crystalline semiconductors with high charge carrier mobilities constitute the main ingredient for most microelectronic devices. Such high degrees of crystallinity and purity are, however, often not desired or feasible due to cost considerations: low-cost thin films of amorphous silicon find, for instance, widespread use in thin-film transistors in the backplane of liquid-crystal displays. In solar cells, amorphous silicon can be processed as cost-saving thin films because 
due to its direct band gap it exhibits a larger absorption as compared to their crystalline counterparts with indirect band gap. Porous oxide nanoparticles form the main constituent in Gratzel-type solar cells (Oregan and Gratzel, 1991). Also, short lifetimes of optically excited charge carriers due to high defect concentrations can be beneficial in certain optoelectronic devices.

It is therefore apparent that "real-life" electronic materials can be found in varying compositions and structural appearances. Ultimately, their electronic properties on the nanometer scale, to which $\mathrm{THz}$ spectroscopy is sensitive to, depend on the degree to which delocalized "ideal" charge transport is disrupted and charge carriers become localized. In the Terahertz probe window the combined response of delocalized and localized charge carriers can lead to intricate spectra. The interpretation of these in terms of a meaningful microscopic charge transport picture remains challenging. In this section we introduce how these effects emerge and how they affect the $\mathrm{THz}$ response.

In order to introduce the role of localization effects and their physical interpretation we compare two studies on silicon, namely, on amorphous and polycrystalline silicon (Cooke et al., 2006; Fekete et al., 2009).

Cooke et al. (2006) compared the $\mathrm{THz}$ conductivities of silicon nanocrystallites embedded in an insulating silicon dioxide matrix ( $\mathrm{Si}-\mathrm{NCs}$, grain sizes 3, 4, and $7 \mathrm{~nm}$ ), nanocrystalline silicon (nc-Si, grain sizes around $20 \mathrm{~nm}$ ), and an epitaxially grown silicon-on-sapphire thin film. The results are shown in Figs. 20(a) and 20(b). The complex conductivity of the silicon-on-sapphire sample shows the typical signatures of a Drude response: a real part that is positive and decreasing with frequency and a positive imaginary part. The crystallite samples however show signs of carrier localization, reminiscent to the response of polymer semiconductors introduced in Sec. III. The real part of the conductivity is positive and decreasing with frequency whereas the imaginary part is negative. The conductivity values at dc are lower, however not vanishing. Clearly, the conduction process over long distances is suppressed by the existence of grain boundaries, most notably by the $\mathrm{SiO}_{2}$ barriers in the $\mathrm{Si}-\mathrm{NC}$ samples.

The study of the effects of disorder on conductivity has a rich scientific history. Generally, there are two regimes of interest: in the "weak" localization, electron motion is still considered diffusive, but electron wave interference caused by coherence after scattering from defects, introduces small deviations from the Drude conductivity. Such effects, first considered by Abrahams et al. (1979), generally only occur at low temperatures in conducting materials. "Strong" localization (Anderson, 1958) occurs when the degree of randomness of the impurities or defects is sufficiently large to inhibit diffusion. A large number of conductivity models have been developed to describe effects due to strong localization. The most well known of these are the variable range hopping model introduced by Mott (1968, 1969), which describes thermally assisted hopping between localized electron sites, and phonon-assisted tunneling of electrons (Ambegaokar et al., 1971), both of which are valid when electrons hop or tunnel a distance greater than the average impurity separation. The Drude-Smith model (Smith, 2001), because of its very general applicability, is a common choice for describing localization effects in $\mathrm{THz}$ measurements. Cooke et al. (2006) applied the Drude-Smith model to describe their data which is given by

$$
\sigma(\omega)=\frac{N e^{2} \tau / m}{1-i \omega \tau}\left[1+\sum_{j=1} \frac{c_{j}}{(1-i \omega \tau)^{j}}\right]
$$

This model was, among others (Mayou, 2000), developed to describe material systems in which long-range transport is suppressed by disorder. It lifts the constraint of the Drude model that charge carriers scatter isotropically, i.e., that their momentum randomizes completely. Instead, charge carriers are allowed to scatter in preferential directions by introducing
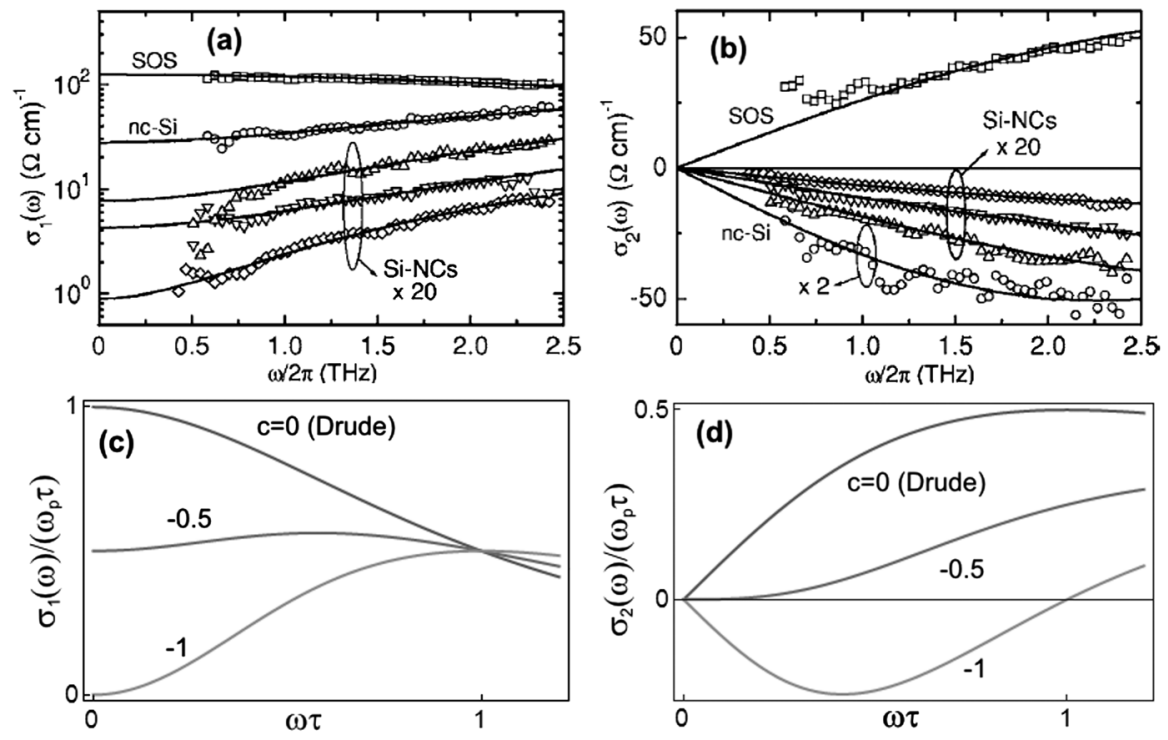

FIG. 20. (a) Real part and (b) imaginary part of the conductivities of photoexcited charge carriers in bulk and nanocrystalline silicon; the lower panels show exemplary plots of the Drude-Smith model for various values of $c$ : (c) real conductivity; (d) imaginary conductivity-both vs frequencies normalized to $\omega \tau$. From Cooke et al. (2006). 
a persistence of velocity parameter $c$ which can have a value between 0 and -1 . It is implemented for every scattering event $j$ in the series term in the bracket of Eq. (12). A key assumption of the model is that the persistence of velocity is retained for only one collision, i.e., only the first scattering event $j=1$ is considered. Despite the crude approximation, this simple empirical model works remarkably well. It can reproduce the signatures characteristic for charge carrier localization, as model plots of the conductivity reveal in Fig. 20(c) (real part) and Fig. 20(d) (imaginary part). $c=0$ yields the common Drude response. A more negative $c$ value increasingly depresses dc conductivity and shifts the oscillator strength to higher frequencies. For $c=-1$, the dc conductivity vanishes completely and the conductivity maximum occurs at $\omega \tau=1$. Translated to the measurements of Cooke et al. (2006), the $c$ parameter can be seen as a measure for the fraction of electrons that bounce back into the grain when they scatter at the boundary and is thus an indicator for the degree of charge carrier localization. The authors obtained values of -0.83 for $\mathrm{nc}-\mathrm{Si}$ and around -0.97 for Si-NCs.

One must note one caveat when describing the $\mathrm{THz}$ conductivity in nanocrystalline materials which are described by a spatially varying conductivity. Such inhomogeneous materials must be treated differently from those that contain large numbers of microscopic local defects, such as impurities or grain boundaries, which are evaluated using scattering theories (Stroud, 1975). This is because a far-field THz measurement reflects an averaged conductivity of a spatially varying material and one has to link the local conductivity in nanocrystalline materials to the average conductivity obtained by far-field $\mathrm{THz}$ measurements. In other words, it is not only the change in local dielectric response that determines the overall dielectric response, but also the contrast in the dielectric response between the material of interest and its surroundings for the unexcited system. To make the connection between the local, spatially varying conductivities and averaged conductivities measured in the far field, requires an effective medium theory, such as those proposed by Garnett (1904) and Bruggemann (1935).

The problem of relating the far-field response to the local conductivity is abated when the dielectric response of the nanomaterial and the surroundings are identical. Such is the case for the experiments of Fekete et al. (2009), who conducted an extensive study on microcrystalline silicon prepared by plasma enhanced chemical vapor deposition. A schematic of the sample structure is depicted in Fig. 21(a). It consists of an amorphous silicon layer in which conically shaped crystallites of around $500 \mathrm{~nm}$ size are embedded. These crystallites in turn are comprised of small grains of several tens of nanometers in size. Conductivity measurements were performed in a temperature range between 20 and $300 \mathrm{~K}$ and modeled with the help of Monte Carlo simulations.

Immediately following photoexcitation, the conductivity resembles a free-carrier response which rapidly vanishes within $0.6 \mathrm{ps}$. This decay is accompanied by the appearance of a conductivity shape characteristic of localized carriers, as shown before. They suggested that upon photoexcitation hot carriers are generated into delocalized bands which hence

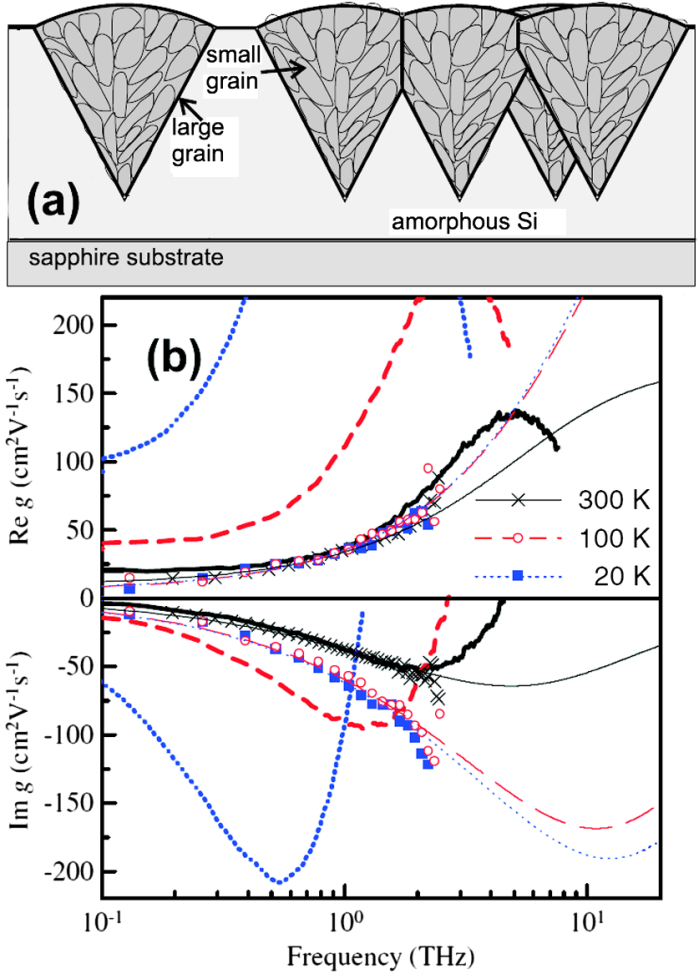

FIG. 21 (color online). (a) Scheme of the composition of the microcrystalline samples under investigation by Fekete et al. (2009), (b) Mobility spectra of charge carriers in microcrystalline silicon for temperatures of 300,100 , and $20 \mathrm{~K}$ at a pump-probe delay of 50 ps. Upper panel: real part, lower panel: imaginary part. Symbols: experimental data, thick lines: Monte Carlo simulations incorporating backscattering, thin lines: fits to hopping model. From Fekete et al. (2009).

exhibit free-carrier properties. The carriers subsequently thermalize with the lattice and get trapped into shallow localized states at the small-grain boundaries within hundreds of femtoseconds.

The mobility of the free carriers at early times was estimated to be only $70 \mathrm{~cm}^{2} / \mathrm{Vs}$ - an order of magnitude lower than bulk values. Furthermore, this mobility value and the free-carrier lifetime were independent of the sample temperature. These facts corroborate the speculation that the signal indeed originates from hot carriers whose temperature was estimated to be $1000 \mathrm{~K}$. Charge carriers at such elevated temperatures experience increased scattering. That is primarily caused by their high kinetic energy rather than the lattice temperature-which explains the insensitivity to the steadystate sample temperature.

In order to understand the localization mechanism, they applied Monte Carlo simulations which included specific microscopic parameters that influence charge carrier transport. At first, they incorporated a charge carrier behavior similar to the Drude-Smith model (i.e., free charge carriers spatially confined within a grain that have a certain probability to be backscattered into the grain) The conductivity at room temperature could be reproduced [see Fig. 21(b), thick line; note that the graphs are plotted in terms of the mobility $g$ rather than the conductivity] assuming grain sizes of 20-30 nm, however, only with a physically unreasonable 
high backscattering ratio of $99 \% .^{3}$ Even more, the theoretically predicted temperature dependence was very different from the experimental results (thick lines).

In a second scenario it was assumed that charge carriers get trapped into shallow localized states which would result in carrier transport dominated by hopping, without a significant Drude-like contribution. In that sense, the peak position of the resonance in the frequency-dependent conductivity can be related to a characteristic frequency on which the conductivity saturates, i.e., on which the hopping process is most efficient. The model results are shown as thin lines in Fig. 21(b). The saturation frequency shifts to higher values at lower temperatures - from $10 \mathrm{THz}$ at room temperature to $22 \mathrm{THz}$ at $20 \mathrm{~K}$. This suggests that a scattering mechanism which is activated at higher temperatures limits the upper hopping frequency and the maximum hopping mobility. It was suggested that scattering with optical phonons (located at $16 \mathrm{THz}$ ) could be the origin. At low temperatures, optical phonons are less populated; the electron-phonon scattering mechanism hence deactivated. This would push the conductivity saturation frequency to values past the optical phonon frequency.

\section{Plasmon resonance}

Bulk plasmons are quantized, longitudinal oscillations of charge which occur in a plasma near the plasma frequency $\omega_{p}$. In a homogenous, three-dimensional material, one cannot couple optically to plasmon modes with a transverse electric field of a plane wave light source, and plasmon modes manifest themselves as nodes, rather than resonances, in the bulk dielectric function of a conductor (Kittel, 1995). Surface plasmons, first predicted in the 1950s by Ritchie (1957), are plasmons which are confined to interfaces between conductors and dielectrics. As is the case for bulk plasmons, surface plasmons at a flat metallic interface cannot be excited by plane wave light, because the wave vector of the plasmons is always larger than the wave vector of the incident light. This momentum mismatch can be overcome by scattering effects on rough surfaces (Kretschmann, 1972), for which coupling to surface plasmons occurs. Similarly, in inhomogeneous conducting materials one can couple to local plasmon oscillations. Semiconductors, on the other hand, exhibit significantly lower plasma frequencies than metals (Isaac, Barnes, and Hendry, 2008; Isaac, Rivas et al., 2008), so that one can expect coupling to $\mathrm{THz}$ plasmon oscillations in structured semiconductors. Such plasmon oscillations produce resonances in the effective dielectric function, similar to those expected from carrier localization effects (see preceding section). However, the physical origin of a plasmon resonance is very different from the single particle, localization effects described in the previous section: plasmon resonance is a many-body effect, and results in a coherent oscillation of all the conducting electrons.

\footnotetext{
${ }^{3}$ Boundaries between small grains as investigated here are commonly due to lattice dislocations. The electronic barriers associated with them are rather low and therefore expected to only cause small perturbations on the drift of charge carriers.
}

To illustrate the origins of plasmon resonance, we consider the work of Nienhuys and Sundstrom (2005a), who measured $\mathrm{THz}$ photoconductivities of isolated silicon microspheres embedded in a polyvinylpyrrolidone matrix. These particles were fabricated by simply grinding a silicon wafer to a powder with particle sizes between 1 and $30 \mu \mathrm{m}$. The conductivities for three different excitation fluences are shown in Fig. 22(a). Since the particles were completely isolated, the system lacked any pathway for long-range charge transport. It is therefore not surprising that the conductivity does not follow the Drude law and vanishes for probe frequencies approaching zero. Notably, the spectra feature a resonance whose center frequency shifts to higher values, proportionally to the square root of the charge carrier density. One should note that the Drude-Smith model cannot account for this behavior; its resonance frequency is solely defined by the scattering time (in addition, this model would not make sense physically: given that the particle dimensions are in the
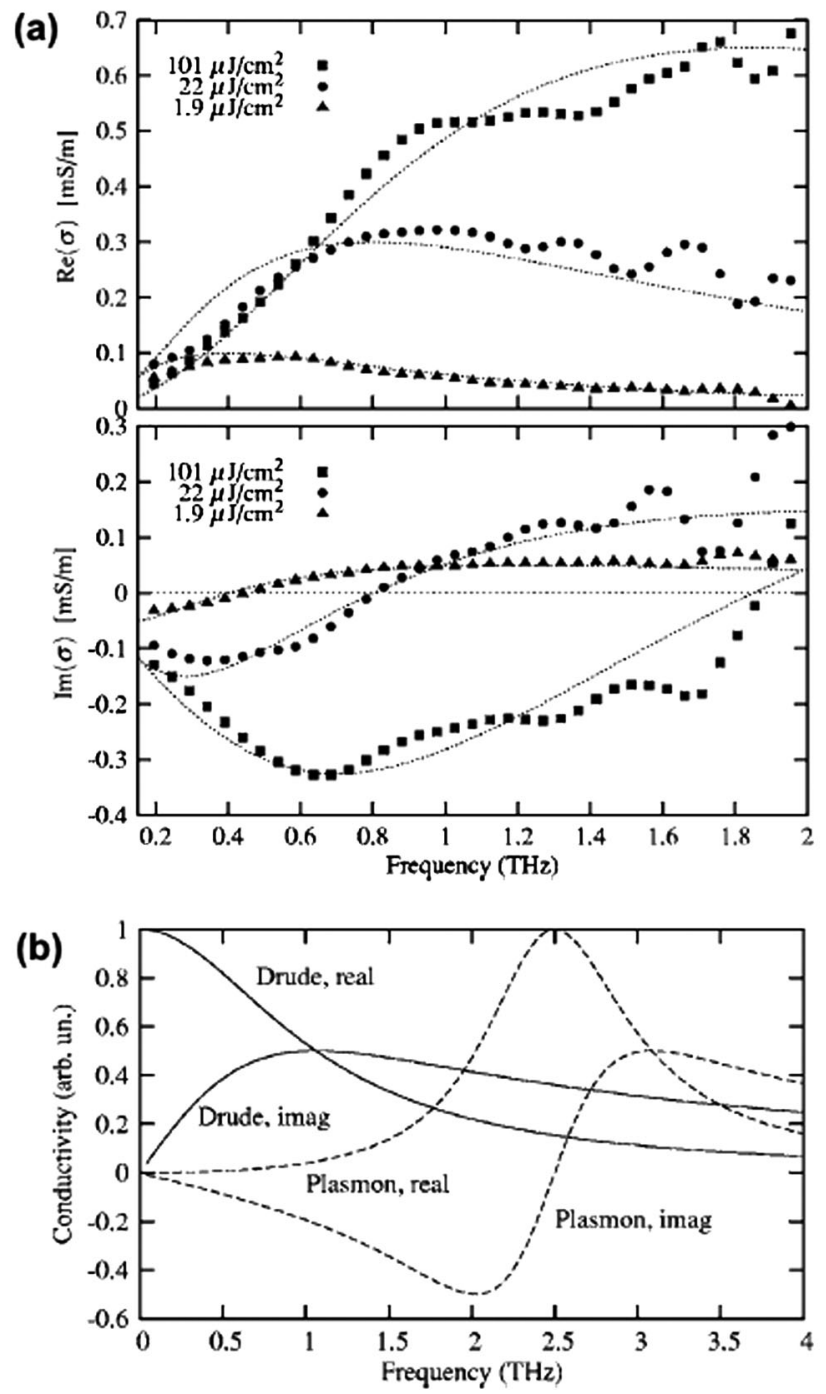

FIG. 22. (a) Complex conductivity of photoexcited charge carriers in silicon microparticles for three pump fluences (upper panel: real part, lower panel: imaginary part) and (b) exemplary conductivity plots of the Drude model and a plasmon resonance. From Nienhuys and Sundstrom (2005a). 
micron range and thus much larger than the charge carrier diffusion length on picosecond time scales, the relative amount of carriers that backscatter from the surface is negligible). A hopping model is also not appropriate: intraparticle transport is still expected to be bulklike, and interparticle transport—or transfer into the polyvinylpyrrolidone matrixis practically impossible. It is therefore clear that these transport models, which describe localization of single particles in an inhomogeneous material, cannot account for the behavior shown in Fig. 22.

To understand the response, it is instructive to recall that the terahertz probe acts as an oscillating electric field that displaces electrons and holes in opposite directions. Because of the restricted size of the particles, charges will accumulate at the particle boundaries and form a space charge layer at the surface. The resulting dipole moment acts as an additional force on the motion of charge carriers (depolarization field) and has thus to be included as a restoring force in the differential equation of the damped harmonic oscillator introduced in Sec. II.E.1 to derive the Drude model. The solution gives a conductivity $\sigma_{\mathrm{pl}}$ which is essentially represented by the Drude model whose resonance frequency has shifted from zero to higher frequencies (see Fig. 22):

$$
\sigma_{\mathrm{pl}}=\frac{N e^{2} \tau_{D} / m}{1-i \omega \tau_{D}\left[1-s\left(\omega_{p}^{2} / \omega^{2}\right)\right]}
$$

$\tau_{D}$ is the Drude scattering time, $s$ denotes a scaling factor that shifts the resonance frequency depending on the particle shape $^{4}$ (in the case of spherical particles as considered here, $s=1 / 3$ ) and the dielectric constant of the particle and the surrounding medium (which were neglected in this study). One should note that Eq. (13) is a Lorentzian resonance [introduced in Sec. II.E.2, Eq. (10)], defined in terms of conductivity, with a resonant frequency $\omega_{0}=\omega_{p} \sqrt{s}$. Hence, the resonance frequency scales with the plasma frequency $\omega_{p}$ and with the square root of the charge carrier density, in accordance with the measured conductivities. The plasmon model for spherical particles as considered above gives the same resonance condition (i.e. $\omega_{0}=\omega_{p} \sqrt{s}$ ) as is obtained by inserting the Drude conductivity [Eq. (9)] into Maxwell-Garnet effective medium theory [Eq. (4)] in the limits $\omega \tau \gg 1 / \tau_{D}, \epsilon_{m}=\epsilon=1$ and the filling fraction $f \rightarrow 0$ (Hendry, Koeberg, O'Regan, and Bonn, 2006). This is because Maxwell-Garnet effective medium theory is derived from the Clausius-Mosotti relation (Feynman, 1970), and therefore takes into account the local depolarization field due to surrounding charge carriers. Indeed, it is well known that, in contrast to the Bruggemann's approximation, Maxwell-Garnet effective medium theory reproduces plasmon resonance at optical frequencies in islandized metals (Riikonen et al., 2005). Strictly speaking, Maxwell-Garnet effective medium theory is correct only to first order expansion in $f$ (Spanier and Herman, 2000), and therefore gives exact results in the limit $f \rightarrow 0$. Despite this limitation, there is convincing evidence (Gittleman and Abeles, 1977)

\footnotetext{
${ }^{4}$ For metal nanoparticles that exhibit plasmon frequencies in the visible this dependence can be nicely seen as a variation of the particle color with particle shape (Murray and Barnes, 2007).
}

suggesting that Maxwell-Garnet theory may also satisfactorily reproduce plasmon resonances observed at optical frequencies in porous or granular metals, and has been shown to produce meaningful results (Granqvist and Hunderi, 1978) even for materials described by $f \sim 0.5$.

In addition to the observation of plasmon resonance in isolated silicon particles described above, there have been several studies where plasmon resonance has been observed in semiconductor nanowires. The diameter of most nanowire semiconductors fabricated today is larger than the exciton Bohr radius and electronic quantum confinement effects are thus not observed. For a general review of charge carrier dynamics in semiconductor nanowires, see Prasankumar et al. (2009). Most nanowires fabricated so far have not yet reached lateral sizes small enough to exhibit quantum confinement effects. Their response in the terahertz region is thus expected to be of plasmonic nature as well. Parkinson et al. (2007) investigated charge carrier dynamics in GaAs nanowires. After photoexcitation, the conductivity vanishes rapidly within 1-2 ps, an indication of severe trapping of charge carriers in surface trap states. Interestingly, the accompanied decrease in charge carrier density is reflected as a redshift of the plasmon frequency within the probe spectrum (see Fig. 23, plasma frequency indicated with arrow). Around $300 \mathrm{fs}$ after photoexcitation the localized surface plasmon mode has build up at a frequency of $1 \mathrm{THz}$. Its peak shifts within the next picosecond to lower frequencies until it is outside the probe window and all charge carriers have been trapped.

In a follow-up paper Parkinson et al. (2009) correlated the influence of growth conditions of GaAs nanowires and their overcoating with a larger-band gap material on the chargecarrier mobility and lifetime.

The influence of depolarization fields on the $\mathrm{THz}$ response of nanowires depends on the sample dimensions with respect to the probe polarization: this was explicitly demonstrated by
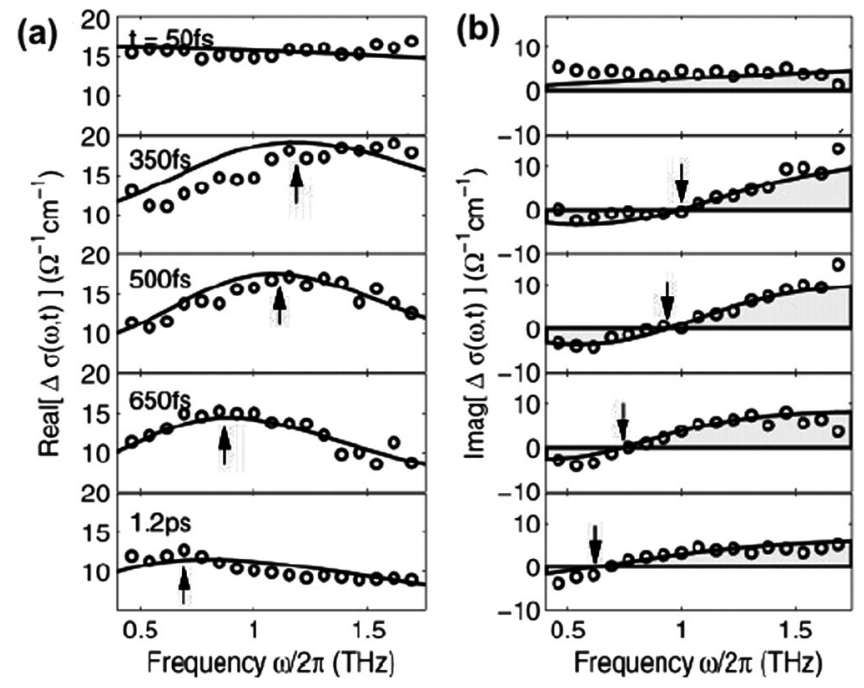

FIG. 23. (a) Real part and (b) imaginary part of the conductivity of photoexcited charge carriers in GaAs nanowires for various pumpprobe delays. A redshift of the localized surface plasmon resonance frequency is observed (indicated by the arrows): trapping at surface states decreases the charge carrier density over time and hence the plasmon frequency. From Parkinson et al. (2007). 
Strait et al. (2009). Germanium nanowires ( $l=10 \mu \mathrm{m}, d=$ $80 \mathrm{~nm}$ ) had been transferred in an aligned fashion from the growth substrate onto quartz. The high degree of ordering is observable in the dark-field optical micrograph image in Fig. 24(a). The sample was oriented in such a way that the $\mathrm{THz}$ polarization had an angle of $45^{\circ}$ with respect to the nanowire axes. A polarizer was used to select certain polarization components, indexed by the angle $\Theta$. An angle of $90^{\circ}$ corresponds to the polarization being perpendicular to the axis whereas for $\Theta=0$ it is parallel [see sketch in Fig. 24(b)]. The dynamics of the photoinduced transmission change are shown in Fig. 24(b). The decay is due to carrier recombination, most likely via surface defect states. The transmission change, i.e., the real conductivity, is largest for polarizations along the nanowire axis and decreases with increasing $\Theta$ until for $90^{\circ}$ it has vanished completely. They applied Eq. (13) to explain the results. For $\Theta=0$, the scaling factor $s$ was set to $(d / L)\left(\epsilon_{s} / \epsilon_{0}\right)^{1 / 2}$. Since $d \ll L$, the scaling factor pushed the plasmon frequencies below $300 \mathrm{GHz}$ and hence below the $\mathrm{THz}$ window for all charge carrier densities. This implies that the conductivity approximately resembles the bulk plasmon mode, i.e., the traditional Drude response with a peak at dc. For polarizations perpendicular to the nanowire axis, $s$ was set to $\left[\epsilon_{s} /\left(\epsilon_{s}+\epsilon_{0}\right)\right]^{1 / 2}$. This yielded plasmon frequencies of tens of THz. Since the width of the plasmon peak is relatively narrow due to the high carrier scattering times in germanium, no absorption can be found in the $\mathrm{THz}$ window.

Two recent investigations report the dark and photoconductivity of nanoporous InP structures (Lloyd-Hughes et al., 2008; Merchant et al., 2008). The samples were fabricated by electrochemical etching of $n$-doped InP wafers, generating pores perpendicular to the surface. A scanning electron microscope image and a schematic are shown in Fig. 25(a).
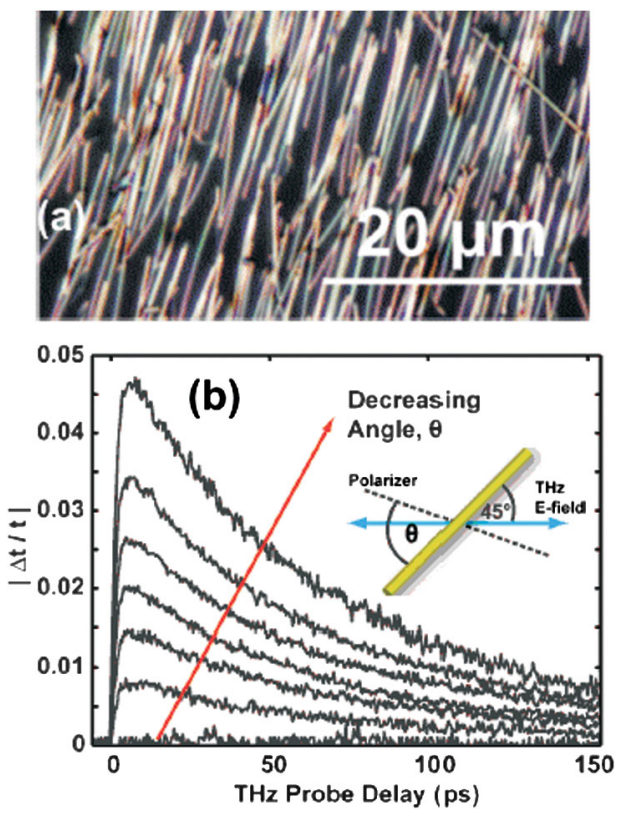

FIG. 24 (color online). (a) Dark-field optical micrograph of oriented germanium nanowires. (b) Measured differential transmission for various polarizer angles $\Theta$. Inset: Sketch of the geometry of nanowire orientation, $\mathrm{THz}$ electric field, and polarizer angle $\Theta$. From Strait et al. (2009).
The pores had diameters of around $60 \mathrm{~nm}$ and were separated by a distance of around $100 \mathrm{~nm}$. The dark conductivities, i.e., of the extrinsic electrons introduced by doping, of three samples with different degrees of porosity featured the common signs of localized charge transport: a real part that is positive and increasing with frequency and a negative, increasing imaginary part. The real part was further suppressed at higher porosities, accompanied by an increased imaginary part. Clearly, a higher porosity impairs charge transport. Interestingly however, the conductivity of photoexcited charge carriers displays a free-carrier response with mobility values that are independent of the excitation density and are of roughly half the bulk value.

In order to explain the observations they calculated the two-dimensional carrier density, assuming an electron doping density of $10^{18} \mathrm{~cm}^{-3}$. Additionally, it was assumed that electronic states at the pore surface pin the Fermi level above its bulk value. The bands thus bend upwards near the pores, creating a surface layer depleted of electrons. The calculated spatial charge carrier density distribution is shown in Fig. 25(b): the carrier densities in regions close to the pore approach zero and are hence of low conductivity. The increasing prevalence of depleted areas in the higher-porosity samples thus explains the decreasing conductivity trend. The localized conductivity response can now be understood by visualizing the areas with a significant amount of charge carriers as completely isolated from each other by depleted zones, hence lacking any percolation pathway between them. These carrier islands exhibit localized surface plasmon modes with plasmon frequencies much higher than the $\mathrm{THz}$ probe bandwidth.

The scenario after photoexcitation is shown in Fig. 25(c). Here the color legend indicates the change in charge carrier

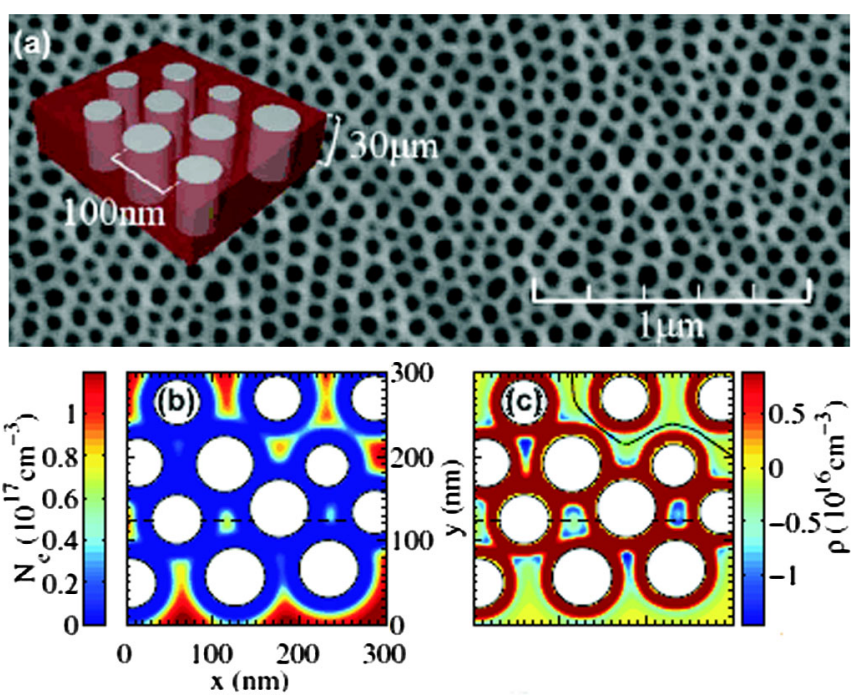

FIG. 25 (color online). (a) Scanning electron micrograph of the surface of nanoporous InP; the inset shows a three-dimensional cartoon of the porres. Simulations of the spatial distribution of the carrier density in nanoporous $\mathrm{InP}$ at $100 \mathrm{~K}$ : (b) Before photoexcitation regions close to the pores are depleted of charge carriers due to band bending. (c) After photoexcitation, the bands flatten out and charge carriers are distributed more homogenously. From Lloyd-Hughes et al. (2008). 
density as compared to the dark conductivity. As can be seen, the charge density in formerly depleted regions close to the pores increases whereas it decreases in formerly populated regions. The band bending has thus been flattened out and charge carriers have been distributed more homogeneously. In fact, no entirely depleted areas can be found anymore. Charge transport is thus well percolated, in agreement with the measured free-carrier response.

\section{Localization versus particle plasmons}

Since plasmon resonance and carrier localization in inhomogeneous semiconductors can both result in conductivity resonances in the $\mathrm{THz}$ frequency region, it is often difficult to determine the physical origin of any resonance behavior in these materials. To illustrate this point, we summarize below two works published in recent years which investigated the $\mathrm{THz}$ response of nanoporous $\mathrm{TiO}_{2}$ films (Turner et al., 2002; Hendry, Koeberg, O’Regan, and Bonn, 2006). These studies illustrated how one can interpret $\mathrm{THz}$ measurements on the same system in different ways.

First published was the work by Turner et al. (2002), who studied the $\mathrm{THz}$ photoconductivity of a porous material composed from sintered $\mathrm{TiO}_{2}$ nanoparticles (25 nm radius). The $\mathrm{TiO}_{2}$ matrix was sensitized with photoactivated dye molecules, from which carriers were injected into the $\mathrm{TiO}_{2}$ matrix. The photoconductivity, measured at $77 \mathrm{~K}$, exhibited a clear resonance in the $\mathrm{THz}$ spectrum. They interpreted this response as a localization effect, fitting their frequencydependent conductivity using the Drude-Smith model (Smith, 2001). This fit yielded a mobility for the porous material of $1.5 \mathrm{~cm}^{2} / \mathrm{Vs}$, compared to the bulk value for $\mathrm{TiO}_{2}$ of $56 \mathrm{~cm}^{2} / \mathrm{Vs}$.

In later work on a similar system, Hendry, Koeberg, O'Regan, and Bonn (2006) also measured the THz photoconductivity of sintered, $25 \mathrm{~nm} \mathrm{TiO}_{2}$ nanoparticles. There were, however, a number of subtle differences from the original experiments by Turner et al. (2002). First, in order to remove effects due to injection, carriers were introduced by directly photoexciting an unsensitized $\mathrm{TiO}_{2}$ with both 266 and $400 \mathrm{~nm}$ light. This means that both electrons and holes were excited in the material. By comparing directly the photoconductivity of the nanoporous material with that measured for a homogeneous $\mathrm{TiO}_{2}$ single crystal (Hendry, Wang, Shan et al., 2004), they were able to determine that the $\mathrm{THz}$ mobility of electrons in the porous sample was $\sim 10^{-2} \mathrm{~cm}^{2} / \mathrm{Vs}$ at $77 \mathrm{~K}$. The contribution from the holes was negligible due to the very large effective mass of holes in $\mathrm{TiO}_{2}$. On increasing the temperature of the sample to $300 \mathrm{~K}$, a reduction in mobility of only a factor of 2 (compared to a change of almost 2 orders of magnitude in the bulk $\mathrm{TiO}_{2}$ material) was observed. More notably, they also observed a conductivity resonance in the $\mathrm{THz}$ conductivity of the nanoporous material. By varying the excitation intensity, they observed that the resonance frequency depended on carrier density, which is a strong indication of a plasmon resonance. They therefore employed Maxwell-Garnet effective medium to describe the observed plasmon resonance. This model also explained the relative insensitivity of the $\mathrm{THz}$ conductivity to temperature, since the plasmon resonance frequency is determined only by the carrier density, and not the scattering rate.
While both Turner et al. (2002) and Hendry, Koeberg, Wang et al. (2006) observed similar resonances in their $\mathrm{THz}$ conductivity spectra of nanoporous $\mathrm{TiO}_{2}$, two different explanations for the physical origin of the response were given: as an intraparticle, single-carrier response governed by boundary scattering, on the one hand, and as a Drude response buried in an effective medium, on the other hand, resulting in a plasmon resonance. This illustrates how $\mathrm{THz}$ conductivities ought to be evaluated with care, as one essentially has to distinguish how local, single-carrier properties (Drude, hopping, backscattering) and local field or manybody contributions (dielectric screening, depolarization fields) affect the overall, far-field response. A proper understanding of both the local intraparticle behavior and local field effects due to inhomogeneities in the dielectric response are thus imperative.

To address the issue of local conductivity, Nemec, Kuzel, and Sundstrom (2009) performed Monte Carlo simulations to calculate the conductivities of charge carriers in spherical particles of varying sizes. Only a few parameters entered the simulation: the particle dimension $d$, the thermal velocity $v_{t h}$, and the carrier momentum scattering time $\tau$ (and hence the mean free path $\left.l_{f}=v_{\mathrm{th}} \tau\right)$. The parameter $p_{r}$ denotes the probability that upon scattering with the particle boundary a charge carrier is reflected back into the particle ( $p_{r}$ is thus akin to the $c$-parameter in the Drude-Smith model). The degree of localization was expressed as the parameter $\alpha$, defined as the ratio between the mean free path and the particle dimension. They concluded that the shape of the conductivities only depend on $\alpha$ and $p_{r}$.

The results for completely isolated particles (corresponding to $p_{r}=1$ and $c=-1$ in the Drude-Smith model) are shown in Fig. 26(a). For $\alpha$ going to infinity, i.e., $d \gg l_{f}$, the conductivity is represented by the Drude model. For $\alpha<10$, the dc conductivity significantly drops and a resonance appears. The center frequency of this peak is related to the ballistic round-trip time of carriers in the sphere $\tau_{\mathrm{rt}}=2 \mathrm{~d} / v_{\mathrm{th}}$. For smaller particles, this round-trip time decreases which is reflected in a blueshift of the resonance frequency for decreasing $\alpha$. Additionally, the conductivity amplitude decreases with $\alpha$. The conductivity evolution is in principle similar to the case of $p_{r}=0.5$ [Fig. 26(b)] with the exception that the real part does not vanish at dc for all values of $\alpha$, proving that long-range transport is enabled.

One should note, however, that the spectra calculated by Nemec et al. (2009) still represent local, single-carrier properties of the material. In order to obtain the effective response of the whole system one would still have to either incorporate carrier-carrier interactions into the calculation or alternatively apply an appropriate effective medium theory. Nevertheless, we still can consider, in a general manner, the effects that carrier-carrier interactions would have: for a nanoporous material in which the interparticle transport is inefficient (i.e., one which exhibits a localized, single particle conductivity resonance), carrier-carrier interactions would introduce an additional restoring force for carriers near the interface, pulling them towards the center of the particle. Such a restorative effect would shift the spectral weight of the low-frequency, localized resonance, to higher frequencies (see Sec. II.E.2). This means that on increasing the strength of 

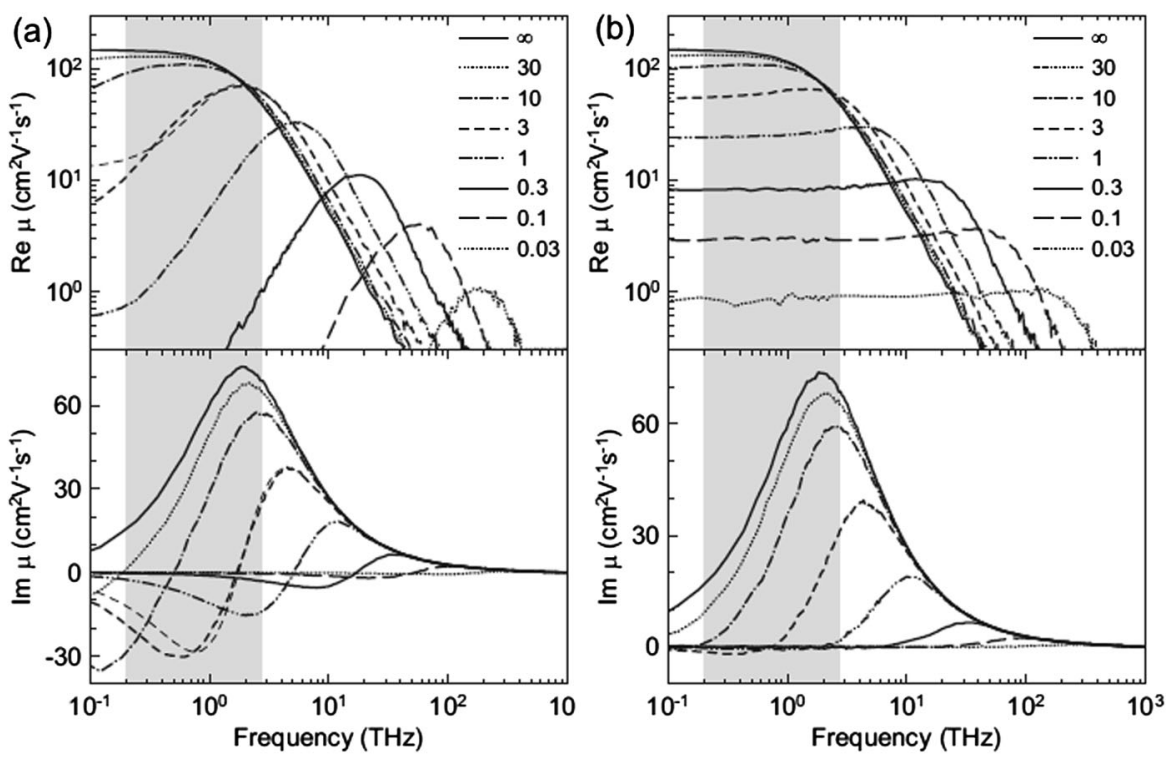

FIG. 26. Mobility spectra of localized particles calculated by Monte Carlo simulations for various values of $\alpha$ (upper panels: real part, lower panels: imaginary part). (a) $p_{r}=1$, (b) $p_{r}=0.5$. From Nemec, Kuzel, and Sundstrom (2009).

carrier-carrier interactions (by increasing the carrier density) one can expect to observe the evolution from a localized resonance to plasmon resonance in the same material. This problem of resolving plasmon resonance from localization effects is obviously not restricted to $\mathrm{TiO}_{2}$ but can also occur in other nanostructured materials that possess domain dimensions comparable to the carrier mean free path.

\section{B. Structures with quantum confinement}

The previous section dealt with nanostructured semiconductors in which the typical dimension of the carrier de Broglie wavelength remained small compared to the physical dimensions of the semiconductor nanostructures. The effects of quantum confinement take place when the size of a system or structure approaches the de Broglie wavelength of the charge carriers (electrons or holes) in one, two, or all three dimensions. Such a structure is referred to as a quantum well, quantum wire or nanowire, and quantum dot, respectively. The electronic and optical properties of these structures deviate substantially from those of their bulk counterparts (Harrison, 2002). Typically, this quantum confinement leads to discrete energy states of carriers (in analogy to a quantum mechanical particle in a box) and, depending on the confinement dimensionality, to various other effects like change in band gap energy or exciton binding energy (Haug, 1994; Davies, 1997; Gaponenko, 1998). This behavior will affect the $\mathrm{THz}$ response; inversely, the $\mathrm{THz}$ response can be used to characterize confined charge carriers, as will be demonstrated in this section for quantum wells, quantum dots, and graphite nanostructures.

\section{Quantum wells}

QWs are often formed in semiconductors by sandwiching a material between two layers of another wider band gap material such as GaAs/AlAs. These structures typically are grown by either molecular beam epitaxy or chemical vapor deposition with a control of the layer thickness down to monolayers. The potential well in the growth direction confines electrons and holes in the plane and creates subbands [see Fig. 27(a)]. Within the subbands, charge carriers behave as a free quasi-two-dimensional electron gas (Shik, 1999).

Initial THz-TDS experiments on QWs measured basic linear properties of intersubband (IS) transitions, e.g., their dispersion (Heyman et al., 1998), induced polarization (Kersting et al., 2000), and subband population dynamics (Bratschitsch et al., 2002). Note that these early experiments did not probe the electric field via electro-optic sampling but by a heterodyne detection scheme in which the interferometric signal between the probe, and a reference pulse was detected with a bolometer or MCT detector. A typical sample consists of a stack of a few dozens of quantum wells grown on a single substrate. A prism is attached to it that efficiently couples the probe light into the sample at a tilted

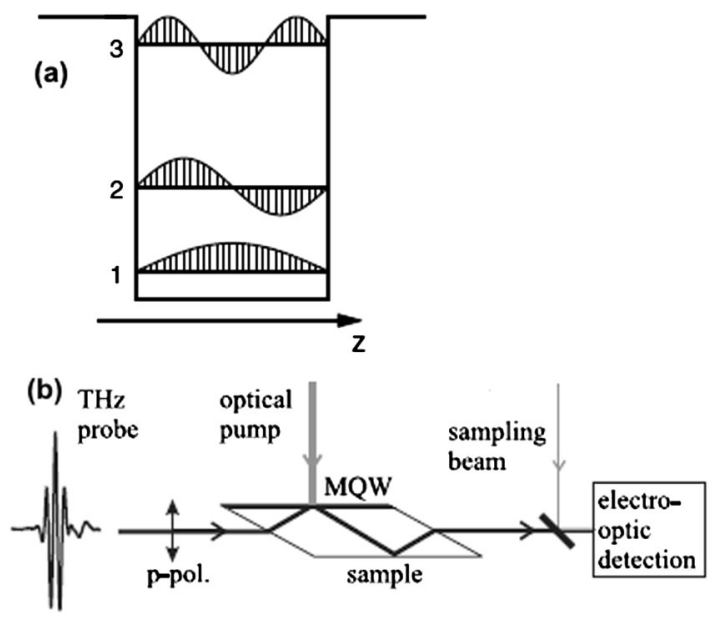

FIG. 27. (a) Sketch of the electron wave functions in subbands of quantum wells; (b) schematic of a optical pump-THz probe experiment on multiple-quantum-wells samples. From Golde et al. (2009) and Luo, Reimann, Woerner, Elsaesser et al. (2004). 
angle [see Fig. 27(b)] In that way a p-polarized THz probe is sensitive to both the in-plane IS transitions and the twodimensional free charge carrier plasma. The interference between the two contributions, manifested as a Fano resonance, was observed only recently in QWs (Golde et al., 2009).

The sample studied consisted of undoped GaAs QWs of $8.2 \mathrm{~nm}$ diameter that featured the $1 \rightarrow 2$ IS transition at 27 THz. A $100 \mathrm{fs}$ optical pump pulse at $1.56 \mathrm{eV}$ was used to populate the first conduction subband and a MIR probe pulse generated in GaSe to measure the response. The pumpinduced differential transmission of the probe field is shown in Fig. 28(a). It features the induced IS polarization with the common exponentially decreasing envelope due to the free induction decay. However, a clear beating can be observed as well. The origin of the beating is revealed in the frequency domain as a broad absorption peak centered at $20 \mathrm{THz}$ next to the IS transition at $27 \mathrm{THz}$ [Fig. 28(b)]. The spectrum features typical signatures of a Fano resonance: an asymmetric line shape with a dip on the low-frequency side. It is caused by the coherent superposition of the polarizations of a two-level system (the IS transition) and a continuum (the 2D electron gas). The interference is destructive on the red side of the IS resonance, hence causing the undershoot; whereas, it is constructive on the blue side, resulting in a broadening. Interestingly, the plasma contribution vanishes in the absorption spectrum, leaving only the bare IS transition [Fig. 28(c)]. This indicates that the plasma contribution is nonabsorptive in the probed spectral range and hence only its phase behavior

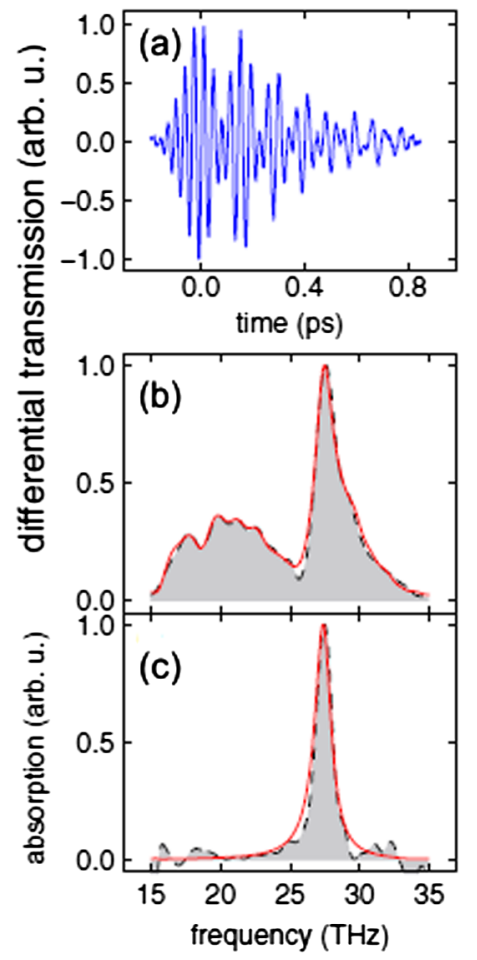

FIG. 28 (color online). Fano effect in intersubband transitions of GaAs quantum wells: the differential transmission of the THz probe field in time shows a beating (a) and a broad peak in the normalized transmission spectrum, additionally to the intersubband resonance (b). The absorption spectrum only features the IS transition (c). From Golde et al. (2009). responsible for the modulation of the overall transmission. This again corroborates the coherent nature of the mechanism.

Transition dipole moments between subbands are comparably strong due to the large extension of the envelope wave function along the $z$ direction. This allows one to drive IS transitions into the nonlinear regime at relatively low electric field strengths (Luo, Reimann, Woerner, and Elsaesser, 2004). As a result, Rabi oscillations can be observed more clearly and at lower electric fields as compared to the case of intraexcitonic transitions (Luo, Reimann, Woerner, Elsaesser et al., 2004). This was observed in $n$-doped GaAs quantum wells that had a charge carrier density such that the Fermi level lies in the gap of the lowest subbands, i.e., only the first subband is populated. The $1 \rightarrow 2$ transition at $24 \mathrm{THz}$ was pumped with a narrow-band MIR pulse generated in GaSe. The dynamics of the reemitted field in the linear regime are similar to the exciton study in Sec. III.B, hence we focus on the nonlinear regime. Figures 29(a) and 29(b) show the
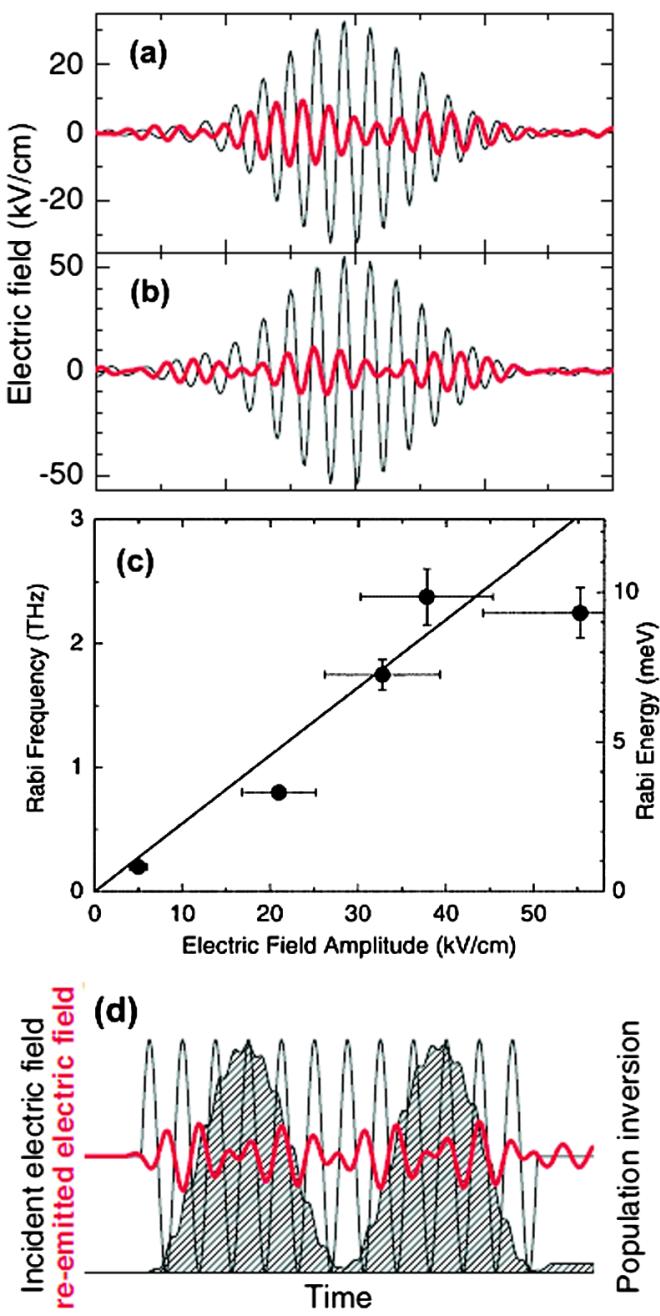

FIG. 29 (color online). Rabi oscillations of intersubband transitions for two different electric-field strengths of the driving field [(a) and (b)]: the thin line indicates the driving field, the thick lines the reemitted field. (c) Rabi frequency as a function of electric-field amplitude. (d) Calculated time evolution of the applied field (thin line), reemitted field (thick line), and population inversion (shaded area). From Luo, Reimann, Woerner, Elsaesser et al. (2004). 
dynamics for driving fields of 25 and $50 \mathrm{kV} / \mathrm{cm}$ in peak amplitude, respectively. The Rabi effect is clearly visible as an envelope modulation of the reemitted field. The time period of that beating decreases for higher driving fields. In fact, the modulation frequency (Rabi frequency) scales linearly with the electric field of the pump pulse [see Fig. 29(c)]. A closer look on the phase relation between the driving field and the reemitted field in each of the Rabi half-cycles reveals the alternating nature of the process between absorption and stimulated emission: a phase difference of $180^{\circ}$, indicative of absorption and hence buildup of population, in one halfcycle, is followed by a phase difference of $0^{\circ}$ in the next half-cycle, caused by stimulated emission and hence depopulation of the excited state. The relation between these parameters is sketched in a simulation in Fig. 29(d).

Apart from the more fundamental scientific insights that quantum wells can provide on quantum phenomena (Wang et al., 2007), recent interest on the application side has been motivated (among others) by their use in quantum-cascade lasers, which consist of a stack of multiple quantum well heterostructures (Williams, 2007). QCLs promise a cheap and compact source for coherent terahertz and MIR radiation. Compared to traditional semiconductor lasers, they rely on a similar design philosophy in the sense that a bias voltage is applied to the structure in order to align the energy bands and create inversion. However, inversion is achieved between intraband (i.e., subband) transitions rather than interband transitions. Furthermore, a recombined electron can be reused by tunneling from the lowest state (injector state) in one QW to the upper laser state (collector state) in the adjacent QW, again building up inversion (cascade; see sketch in Fig. 30(a)]. For more information about the device architecture, see Williams (2007).

A time-domain technique like electro-optic sampling is perfectly suited to unambiguously study lasing dynamics in a phase-resolved manner since it allows one to detect the relative phase between probe and emitted fields. Kroll, Darmo et al. (2007) studied the polarization dynamics of AlGaAs/GaAs $\mathrm{THz}$ QCLs that lased at a frequency of 2.9 THz. The $\mathrm{THz}$ probe pulse was generated by a photoconductive antenna, coupled into the QCL waveguide and its transmission detected by EO sampling. Figure 30(b) shows the electric field after transmission through an unbiased sample. It features the input pulse followed by small oscillations due to weak absorption in the waveguide. The situation changes when a bias is applied, as depicted in Fig. 30(c) which shows the difference signal between unbiased and biased signal. It is now a result of changed absorption (the first single-cycle pulse) and gain (the subsequent oscillation lasting several picoseconds). The transition from initial absorption to stimulated emission can be recognized by a phase jump between the initial pulse and the oscillating part, indicated by the arrow. This behavior is visible in the frequency domain as well: the absorption spectrum in Fig. 30(d) shows a broad absorption peak between 1.0 and $2.2 \mathrm{THz}$, which is caused by the differences in the charge carrier distribution between unbiased and biased conditions. A narrow negative absorption peak, i.e., gain, is observed at the lasing frequency of $2.9 \mathrm{THz}$. It is accompanied by a flipped phase behavior, as compared to absorption, around the resonance which displays

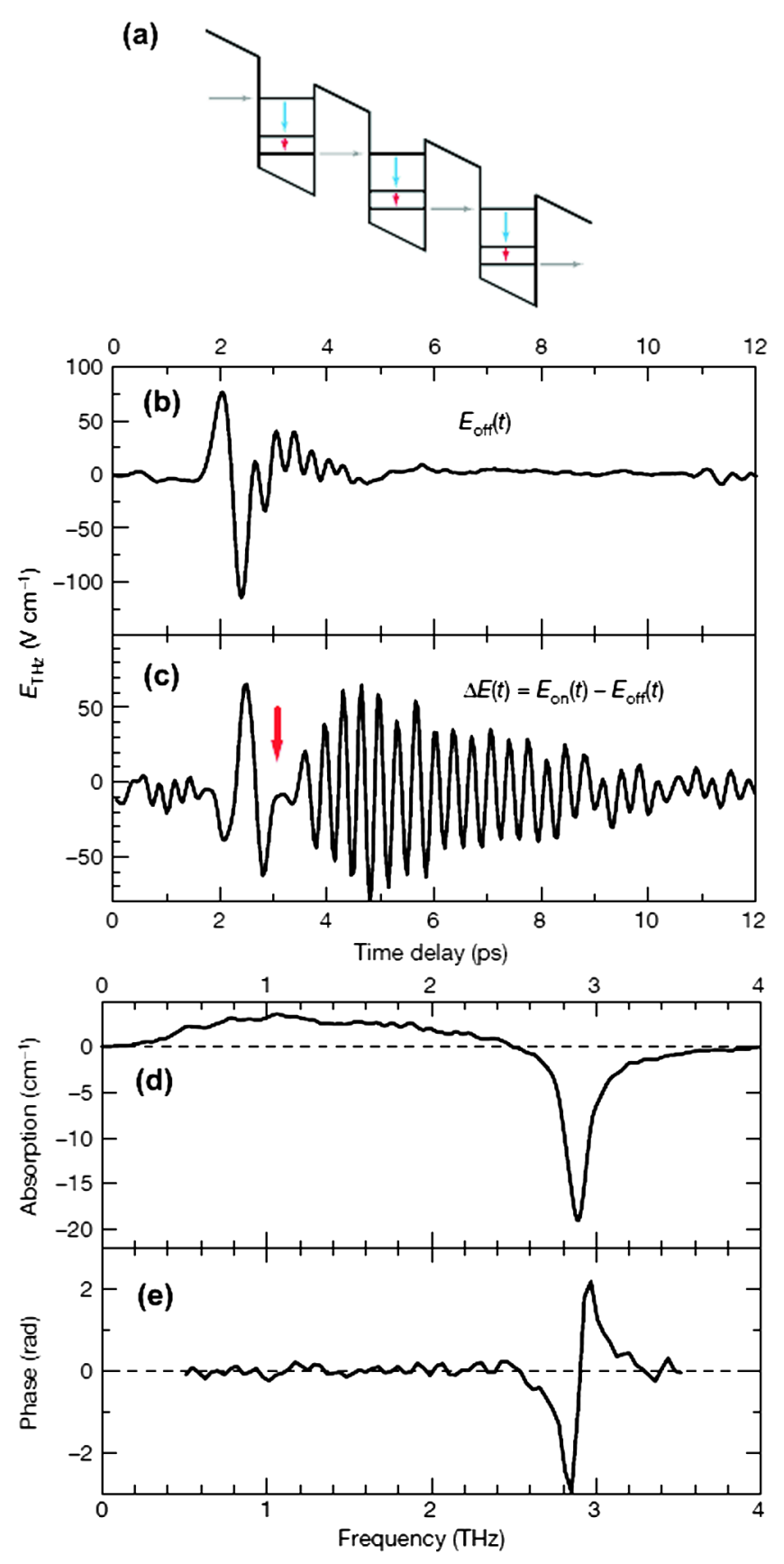

FIG. 30 (color online). Terahertz time-domain spectroscopy of a $\mathrm{THz}$ quantum-cascade laser: (a) Schematic of a quantum-cascade laser; (b) $\mathrm{THz}$ probe transmitted through an unbiased QCL; (c) change in the transmitted probe through a biased QCL; (d) absorption spectra, and (e) phase of the bias-modulated probe. From Kroll, Dhillon et al. (2007).

the negative part on the low-frequency side. This is an unambiguous evidence for stimulated emission.

Other THz studies on QCLs focused on gain dynamics below and above lasing threshold (Kuehn et al., 2008; Jukam et al., 2009), the charge carrier mobility and density (LloydHughes et al., 2009), observed spatial hole burning (Kroll, Darmo et al., 2007), spectral gain narrowing (Jukam et al., 2008), and identified the charge injection process from the injector layer into the upper laser state as being coherent, i.e., through resonant tunneling (Eickemeyer et al., 2002). 


\section{Quantum dots}

Quantum dots are semiconductors with charge carriers confined in all three dimensions (Efros and Rosen, 2000; Harrison, 2002). In QDs the electron and hole energy states become discrete due to the 3D spatial confinement. As a result, QDs have optical responses very different from the bulk material from which they are made. What is more, the QD optical response is tunable: by varying their size (and therefore degree of electronic confinement), the band gaps of QDs can be modified and tuned to cover the entire visible to near-infrared spectral range (Woggon, 1997). As such, they are promising materials for a variety of electro-optic applications, such as LEDs and photovoltaic devices. Knowledge of the processes immediately following photoexcitation is therefore essential, and there has been much interest recently in exciton dynamics in QDs [see Klimov (2007), and references therein], especially in exciton decay, exciton cooling, multiexciton dynamics, and the possible formation of multiexcitons by carrier multiplication.

Many studies of carrier dynamics in semiconductor quantum dots have been performed using ultrafast optical spectroscopy (Klimov, 2007). Most of these studies, typically based on transient absorption or time-resolved fluorescence, have focused on interband transitions in the optical range. The observed optical dynamics in the visible are dictated by both electron and hole dynamics. Infrared transient absorption measurements have been used to study specifically electron dynamics by probing electron intraband transitions. As we will show in the following, $\mathrm{THz}$ spectroscopy has proven to be a fruitful approach for the study of exciton and carrier dynamics in quantum dots and quantum dot assemblies.

In quantum dots, the spacing of the discrete electron and hole energy levels is strongly dependent on the size of the quantum dot. The two relevant length scales of the problem are the QD diameter $D$ and the exciton Bohr radius $a_{B}$ that is the natural physical separation in a bulk crystal between an electron in the conduction band and its corresponding hole in the valence band. Strong confinement in small QDs $\left[D<a_{B}\right.$, see Fig. 31(a)] results in high confinement energies and relatively large energy spacing between the levels, while larger QDs have more closely spaced energy levels. For even larger QDs [ $D \gg a_{B}$, see Fig. 31(b)], the confinement is weak, and charge carriers can move within the boundaries of the QD volume. For assemblies of quantum dots, the electron or hole wave functions between different QDs can couple, and energy and charge transfer can occur within the system [Fig. 31(c)].
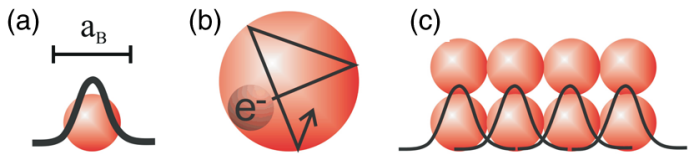

FIG. 31 (color online). Different regimes of charge carriers in quantum dot (assemblies): (a) In the limit of strong confinement, the QD radius is smaller than the exciton Bohr radius and strong confinement occurs. (b) For weak confinement, charge carriers can move within the confined space of the nanocrystal. (c) For QD assemblies, electronic coupling between adjacent quantum dots (owing to the finite overlap of charge carrier wave functions) allows for finite electrical conductivity over macroscopic distances.
In the limit of strong confinement, the energy-level spacing depends on the degree of confinement, but also on the band masses of the electrons and holes: a rule of thumb is that the heavier the quasiparticle the more closely spaced the energy levels. In quantum dots made of II-VI materials such as $\mathrm{CdSe}$, the hole band mass is several times that of the electron, so that the valence electronic levels are more closely spaced than the electron levels [see Fig. 32(a)].

$\mathrm{THz}$ spectroscopy was first used to investigate the nature and dynamics of photoexcitations in semiconductor quantum dots by Beard et al. (2002a), for colloidal CdSe quantum dots of various sizes. They note that for QDs in the strong confinement regime $(<5 \mathrm{~nm}$ diameter $)$, the photoinduced change in the $\mathrm{THz}$ dielectric function is purely real (finite imaginary conductivity; zero real conductivity) and the magnitude increases with the fourth power of the particle radius.

The origin of such a response (purely real dielectric response of photoexcited QDs) was subsequently revealed by Wang et al. (2006) and Dakovski et al. (2007). They argued that for QDs with strong confinement both electrons and holes occupy discrete energy levels separated by at least tens of meV (Efros and Rosen, 2000), i.e., energies significantly larger than the photon energies used to probe the levels $(1 \mathrm{THz}=4 \mathrm{meV})$; see Fig. 32(a). This means that $\mathrm{THz}$ photons with energy below the intraband transitions of electrons and holes interact with with them nonresonantly. The nonresonant nature of the interaction dictates that no absorption of THz light can occur, which explains the experimental observation of a photoinduced change in the $\mathrm{THz}$ dielectric function that is purely real (imaginary conductivity). The amount of photoinduced increase in the dielectric response can be directly related to the polarizability $(\alpha)$ of the exciton.

In a QD, the energy levels of electron and holes scale with the quantum dot radius $R$ as $\propto R^{-2}$, while the Coulomb interaction scales as $\propto R^{-1}$. In the limit of strong confinement, i.e., small $R$, the confinement energies far exceed the Coulomb interactions and the electron and hole comprising an exciton are therefore largely uncorrelated (Efros and Rosen, 2000). Consequently, one can treat the exciton polarizability as the sum of separate contributions from the electron and the hole. The polarizability is a measure of the extent to which the electron and hole wave functions are deformed in an applied electric field. This deformation requires mixing of higher lying states, and occurs more readily when these states are energetically close by. Owing to smaller energylevel spacing, the hole therefore dominates the polarizability at frequencies below the intraband transition energies, giving rise to a real and spectrally flat susceptibility [see Fig. 32(b)]. Typically, the magnitude of the hole polarizability is around $10^{4} \AA^{3}$, scaling as $\alpha \propto R^{4}$ [see Fig. 32(c)]. A more detailed analysis, taking into account the nonparabolicity of the electronic bands and the electron-hole interaction, yields $\alpha \propto$ $R^{3.6}$. Subsequent studies on PbSe quantum dots (Dakovski et al., 2007) revealed that similar conclusions can be drawn for $\mathrm{PbSe} \mathrm{QD}$ systems. However, the $\mathrm{THz}$ response is no longer dominated by the holes due to the comparable band masses of electron and hole and corresponding similar level spacing for conduction and valence states in lead salts.

In comparison, by investigating the response in the range of $2-7 \mathrm{THz}$ of negatively charged CdSe QDs in solution, 

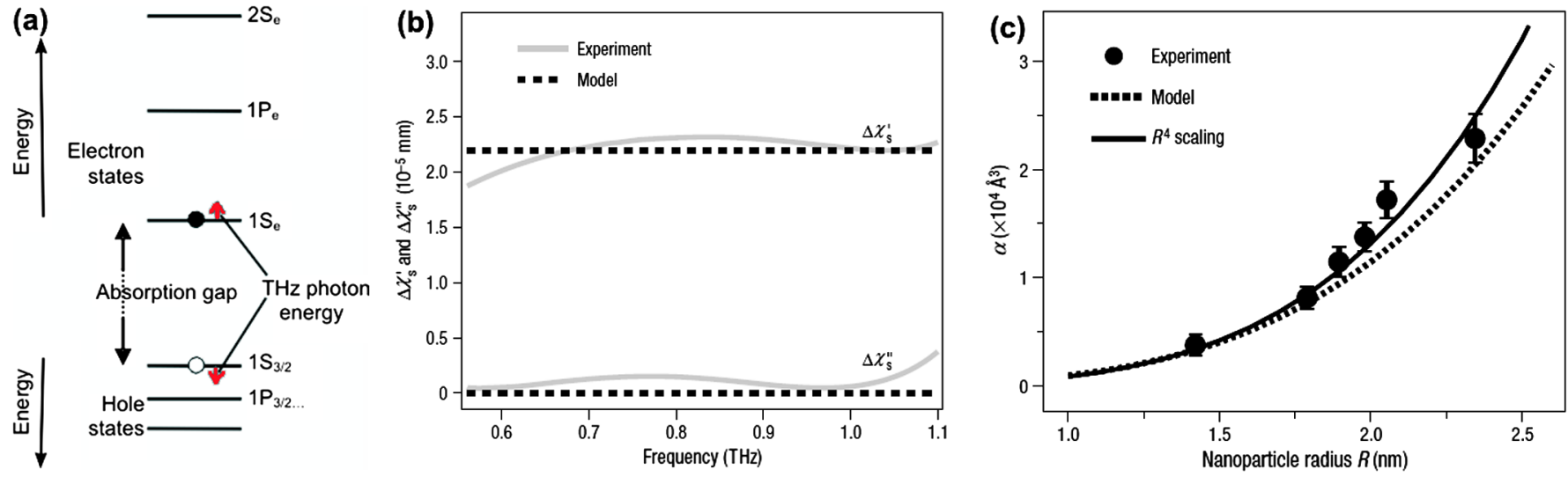

FIG. 32 (color online). (a) Energy-level scheme for a QD in the limit of strong confinement. All transitions occur at energies exceeding that of the low-frequency $\mathrm{THz}$ spectrometer (small arrows). As a result, the excitonic transitions are interrogated off-resonant. (b) The resulting change in the real $\Delta \chi^{\prime}$ and imaginary $\Delta \chi^{\prime \prime}$ parts of the photoinduced sheet susceptibility of a CdSe QD dispersion. The light lines are experimental data and the dashed line represents a purely real, frequency-independent change in the susceptibility that corresponds to purely imaginary conductivity. (c) Polarizability of single excitons in photoexcited CdSe QDs as a function of the QD radius. The experimental data (circles) are well described with a simple $R^{4}$ scaling (solid line). The QD polarizability was calculated by perturbative multiband tightbinding calculations (dashed line). From Wang et al. (2006).

Mandal and Chikan (2007) were able to experimentally determine the electron polarizability in 3.2 and $6.3 \mathrm{~nm}$ diameter QDs to be $0.5 \pm 0.1 \times 10^{3}$ and $14.6 \pm 0.3 \times 10^{3} \AA^{3}$, respectively, around an order of magnitude smaller than the corresponding polarizabilities of the holes.

THz TDS is evidently capable of selectively probing the hole populations in II-VI (e.g., CdSe) quantum dots. By monitoring the hole polarizability as a function of time after excitation, one can evaluate population dynamics of the hole states. This principle has been exploited by Pijpers et al., $(2007,2008)$ to study carrier multiplication in highly excited, small gap, InAs QDs. Moreover, coupling femtosecond optical pump-THz probe measurements with ultrafast optical spectroscopies (such as time-resolved luminescence or transient absorption) can therefore help separate hole from electron dynamics, which is generally challenging. Hendry, Koeberg, Wang et al. (2006) employed a $400 \mathrm{~nm}$ optical pump-THz probe scheme, coupled with time-resolved, band gap luminescence measurements, to extract cooling rates of photoexcited hot holes and hot electrons in colloidal CdSe QDs. These cooling rates are inferred from the rise times of the experimental signals $\tau_{\text {rise }}$, for various QD diameters; see Fig. 33. A clear step in the luminescence rise time is observed

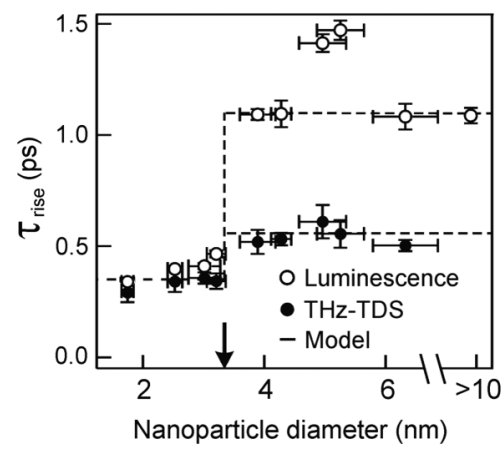

FIG. 33. Cooling times of CdSe nanoparticles as a function of mean particle diameter. From Hendry, Koeberg, Wang et al. (2006). for diameters $>3.5 \mathrm{~nm}$. For particles larger than this diameter, the excitation photon energy $(3.2 \mathrm{eV})$ is sufficient to excite electrons into the $1 P$ excited state, which subsequently relax into the $1 S$ state. The delayed appearance of ground state electrons is reflected in a slower rise of the time-resolved photoluminescence response. Interestingly, a simultaneous slowing down in the $\mathrm{THz}$ response is also observed. This observation of delayed hole cooling upon higher electronic excitation signifies energy transfer from electrons to holes (the holes are reexcited upon electron cooling, resulting in an apparent slowing down of hole cooling). These observations constituted the first direct evidence of electron-to-hole (or "Auger") energy transfer as a key electron cooling mechanism in QDs. A quantitative analysis of the data using a simple rate equation model (lines in figure), allowed for the determination of hole cooling and electron-to-hole energy transfer times of $330 \pm 50 \mathrm{fs}$ and $1 \pm 0.15 \mathrm{ps}$, respectively.

While for CdSe QDs in the strong confinement regime $(<5 \mathrm{~nm}$ diameter), the photoinduced change in the $\mathrm{THz}$ dielectric function was observed to be purely real (finite imaginary conductivity; zero real conductivity), in the weak confinement regime (>5 nm diameter), a complex dielectric response was observed (finite imaginary and real conductivity). Beard et al. (2002a) interpreted the results for the larger particles in terms of a modified Drude transport theory, adapted to include a term accounting for surface scattering.

Larger conductivities have been observed for selfassembled or arrayed (Muller et al., 2008) QD assemblies. The use of THz to study QD assemblies is motivated by the notion that THz TDS is very sensitive to the degree of electronic coupling between the quantum dots (Beard et al., 2003; Crooker et al., 2003; Cooke et al., 2004; Murphy et al., 2006). Strong coupling will allow electrons or holes to "hop" between QDs, giving rise to a real component of the complex conductivity (or equivalently an imaginary component of the permittivity or susceptibility) even at frequencies below the intraband transitions of isolated QDs. Initial studies on quantum dot molecules (Boucaud et al., 
2000), consisting of two vertically coupled InAs selfassembled quantum dots separated by a GaAs barrier, revealed the electronic coupling between the dot states through an intraband absorption at $\mathrm{THz}$ frequencies. In laterally ordered chains of self-assembled InGaAs quantum dots (Cooke et al., 2004), a large anisotropy in the transient photoconductive response was observed depending on the polarization of the $\mathrm{THz}$ probe pulse with respect to the orientation of the dot chains, indicating that the increased $\mathrm{THz}$ response indeed arises from the interdot coupling.

In arrays (or QD solids), THz TDS allows both the degree of interdot electronic coupling and the carrier dynamics (affected by the delocalization of carriers between dots) to be extracted simultaneously. In disordered arrays of $3.2 \mathrm{~nm}$ diameter InP QDs, Beard et al. (2003) reported a sixfold increase in the transient photoconductivity when the average QDs are separated by $0.9 \mathrm{~nm}$ compared to arrays with $1.8 \mathrm{~nm}$ separation (the different separation is introduced by modifying the capping layer of the QD). This change in the transient response was interpreted using the Drude-Smith transport model (see Sec. IV.A), a derivative of the Drude model which takes into account varying degrees of charge localization. By varying pump and probe delays, Beard et al. (2003) also found triexponential decay kinetics for the photospecies, with longer trapping times observed for closer spacing, attributed to the increased tunneling probability between closely spaced QDs, allowing carriers to escape from trap cites. Similarly, Murphy et al. (2006) observed similar effects in PbSe QD arrays.

\section{Graphitic nanostructures}

As one of the most widespread and versatile elements, carbon has been known and studied for centuries. With the discovery of fullerenes, carbon nanotubes, and lately graphene, interest in graphitic materials among the scientific community has been revived in the last several decades. A vast and still expanding volume of information on their physical, chemical, and other properties has been accumulated (Pierson, 1993; Reich et al., 2004; Jorio et al., 2008; Kruger, 2008).

Graphene, a single sheet of $s p^{2}$-hybridized carbon atoms arranged in a hexagonal lattice, is the basic building block for all graphitic materials. It is the latest carbon allotrope to be discovered, having been first experimentally produced and identified only seven years ago by Novoselov et al. (2004). Since then, the worldwide research activities on graphene have grown dramatically. Remarkable fundamental effects have been discovered in graphene (Castro Neto et al., 2009), which are related to its unusual band structure: monolayer graphene is a gapless semiconductor in which charge carriers behave like massless Dirac fermions (Wallace, 1947). Such particles are described by a linear dispersion relation between energy and momentum near the $K$ point of the Brillouin zone 34 (Castro Neto et al., 2009). This unique electronic structure also gives rise to several interesting optical properties: for example, the universal optical absorption of graphene has been shown to be wavelength independent ( $\cong 2 \%-3 \%$ per layer) over a remarkably broad range of optical frequencies (Mak et al., 2008).
All other graphitic materials can be derived from graphene. For instance, monolayer graphene can be stacked up in a crystalline order to form bulk graphite. These structural alternations lead to the modification in the electronic band structure and the corresponding electronic and optical properties of the graphitic materials. In graphite, the band structure is altered by interlayer interactions, leading to the emergence of hyperbolic bands. Graphene can also be rolled up to form carbon nanotubes. By changing the manner in which the edges of the graphene layer join to form the tubes, nanotubes may be engineered to be a semimetal (like graphene) or semiconductor. These one-dimensional (1D) materials exhibit markedly enhanced Coulomb interactions, which is associated with many phenomena in the charge transport of nanotubes, including Coulomb blockade (Bockrath et al., 1997; Tans et al., 1997), Kondo effects (Nygard et al., 2000; Odom et al., 2000), and Luttinger liquid behavior (Bockrath et al., 1999; Ishii et al., 2003). In contrast to graphene, the enhanced Coulomb interactions in 1D carbon nanotubes mean that excitons dominate their optical properties (Wang et al., 2005; Jorio et al., 2008; Geim, 2009).

Photoexcited species play an important role in the electronic and optical properties of graphitic materials. Understanding their nature and dynamics is not only fundamentally interesting, but also important for their applications in electronics, photonics, and photovoltaics. A key question concerns the product of photoexcitation, for instance, in nanotubes (NTs). Coulomb interaction is strong in NTs because of its 1D nature. Whether excitons or free charges are the primary photoexcitation products is still a matter of much debate. Optical pump-THz probe spectroscopy, as we discuss below, provides an ideal tool to resolve this issue by directly measuring both the real and imaginary parts of the optical conductivity over a broad $\mathrm{THz}$ spectral range (Perfetti et al., 2006; Beard et al., 2008; Xu et al., 2009). Another key issue concerns the ultrafast cooling and recombination dynamics of photoexcited electrons in graphitic materials. Optical pump$\mathrm{THz}$ probe spectroscopy with a ps to sub-ps temporal resolution is also a suitable tool to address this question. As we discuss below, THz can be used as a sensitive and contact-free probe to the electronic temperature and the carrier density (Kampfrath et al., 2005; George et al., 2008; Choi et al., 2009). Nevertheless, the technique has a significant drawback in its spatial resolution (a few hundred microns in the far field for low-frequency $\mathrm{THz}$ probes) due to the long-wavelength nature of the $\mathrm{THz}$ radiation. Application of the technique to graphene films has been limited to epitaxial graphene for which large films with a relatively constant layer thickness can be obtained. For NTs, ensembles are typically studied. Although some success has been made in separating singlewalled carbon nanotubes (SWNTs) of a single chirality, practically all the ensembles are of mixed chiralities. The richness of the electronic structure of NTs, on the one hand, provides a wide range of interesting electronic and optical properties, but, on the other hand, makes the interpretation of the experimental observation challenging. Progress has been made towards the measurement of a single NT using the $\mathrm{THz}$ time-domain technique. For instance, the ballistic propagation speed of electrons was measured along a SWNT by integrating a $\mathrm{THz}$ source and carbon NT transistor on the 
same substrate (Zhong et al., 2008). THz electric-field pulses are generated locally by the source and detected locally by the NT transistor.

A variety of graphite and graphene films has been investigated by the $\mathrm{THz}$ method, including thin films of graphite exfoliated from a high-quality crystal of highly oriented pyrolytic graphite and epitaxial graphene grown on $\mathrm{SiC}$ of thickness ranging from a few to monolayers (George et al., 2008; Choi et al., 2009). The equilibrium conductivity of these materials over a broad $\mathrm{THz}$ spectral range was measured. It was found to be consistent with intraband and interband transitions of a dense 2D electron plasmon (Choi et al., 2009). In the low-frequency range $(<3 \mathrm{THz})$ the $\mathrm{THz}$ response is dominated by intraband transitions (see Fig. 34) and can be described by the simple Drude model with the carrier scattering rate and the plasma frequency as parameters. Scattering times ranging from 10s (George et al., 2008) to 100s of fs (Kampfrath et al., 2005) were extracted for various samples at room temperature. The scattering time is controlled by electron scattering with phonons, lattice defects, and other electrons. At higher $\mathrm{THz}$ frequencies, however, contribution from interband transitions in the conductivity is important, particularly, for $\mathrm{THz}$ photon energies around and above twice the Fermi energy.

The first optical pump-THz probe study of graphitic films was performed by Kampfrath et al. (2005), in which a THz probe of $10-25 \mathrm{THz}$ was employed to study the role of strongly coupled optical phonons (SCOP) in the ultrafast carrier cooling dynamics in graphite. It has been proposed that photoexcited electrons rapidly thermalize by transferring most of their energy to SCOPs, which subsequently cool down through anharmonic coupling to other phonon modes. Kampfrath et al. (2005) modeled their experimental pumpinduced complex dielectric function as a sum of intraband and interband transitions (see Fig. 34). The intraband transitions were described by the Drude model and the interband transitions were calculated using the graphite band structure and transition matrix elements between states near the Fermi energy. The electrons were assumed to follow a Fermi-Dirac distribution with an elevated electronic temperature $T_{e}$ after thermalization. Two opposite effects are expected: the intraband transitions enhance the $\mathrm{THz}$ absorption because of the photoexcited carriers and the elevated temperature; the interband transitions, on the other hand, reduce the THz absorption due to state blocking. The temporal evolution of the parameters including the electronic temperature, the carrier scattering rate, and the plasma frequency was extracted by fitting experiment to the model. They concluded that more than $90 \%$ of the initially deposited excitation energy is transferred to a few SCOPs immediately after thermalization; the carrier scattering rate increases from $10 \mathrm{THz}$ at equilibrium to $14 \mathrm{THz}$ shortly after photoexcitation resulted from scattering from hot SCOPs. The slow decay of $T_{e}$ with a time constant of $7 \mathrm{ps}$ was interpreted as the SCOP lifetime, which is compatible with the value of $2 \mathrm{ps}$ from a direct measurement based on the time-resolved Raman spectroscopy (Yan et al., 2009).

Most $\mathrm{THz}$ studies of graphene made use of $\mathrm{THz}$ probes of relatively low frequencies $(<3 \mathrm{THz})$. In this regime the ultrafast optical pump typically reduces the amplitude of

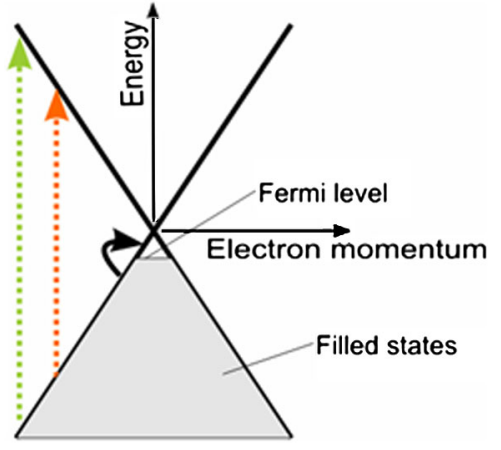

FIG. 34 (color online). Schematic representation of the graphene band structure. Dotted arrows: interband transitions. Solid bent arrow: intraband transitions.

the $\mathrm{THz}$ electric-field waveform transmitted through the photoexcited graphene sample, but without any measurable shift in time or distortion in the waveform. In the frequency domain this pump-induced response corresponds to a dispersionless real conductivity, which is a special case of a Drudelike behavior when the carrier scattering rate is much higher than the probing frequencies. George et al.(2008) and Choi et al. (2009) investigated few-layer epitaxial graphene grown on the carbon face of semi-insulating $6 \mathrm{H}-\mathrm{SiC}$ wafers. The decay of the pump-induced conductivity in their measurement lasted for a few picoseconds and could not be described by a single exponential. They attributed it to the electron-hole interband recombination and proposed plasmon emission, phonon emission, and Auger scattering as possible recombination mechanisms. To account for the detail of the decay dynamics, they had to assume an electron-hole recombination rate that is quadratic with the carrier densities. A similar study was performed by Choi et al. (2009) in thinner epitaxial graphene samples of monolayers and multilayers grown on the $\mathrm{Si}$-face of $\mathrm{SiC}$ wafers, but a single exponential decay was observed for the pump-induced conductivity. Choi et al. (2009) found their samples to be highly $n$-doped and derived that the pump-induced conductivity is dominated by the minority carriers. The observed single exponential decay, therefore, corresponds to the recombination of holes and a largely excitation independent lifetime of 1.2 ps was extracted. This value of recombination time is consistent with Auger and phonon-mediated recombination (Rana et al., 2009).

Next, we turn our discussion to the THz studies of carbon nanotubes. The spectrum of complex conductivity up to $1 \mathrm{THz}$ of SWNT films in equilibrium was first measured by Jeon et al. (2002, 2003). A large anisotropy between the direction parallel and perpendicular to the NT axis was observed in aligned NT films. A more comprehensive study of the equilibrium far-IR properties of SWNTs was reported by Kampfrath et al. (2008). The complex dielectric response or conductivity over a broad spectral range (1-40 THz) was measured for films of high pressure CO grown NTs (Fig. 35). The measurement was accomplished by making use of three different pairs of emitter and detector crystals in combination with laser pulses of $10 \mathrm{fs}$ in duration. The response can be described by a Drude component and a broad Lorentz component peaked around $4 \mathrm{THz}$. The origin of the broad 


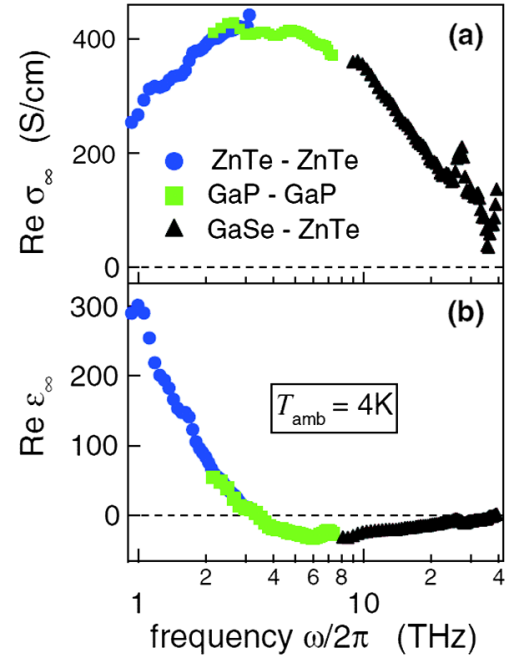

FIG. 35 (color online). Complex dielectric response of unexcited films of high pressure CO grown NTs. From Kampfrath et al. (2008).

absorption peak, however, is not well understood. Interband transitions in small gap SWNTs have been proposed. These are very small SWNTs that have their chiralities satisfying the criterion for metallic tubes, but a small gap in the range of $10 \mathrm{meV}$ is induced by the curvature of their tube walls. However, the resonant feature shows a very weak temperature dependence, uncharacteristic for electronic transitions at such low energies. Phonons and plasmons have thus been proposed as alternative mechanisms. Kampfrath et al. (2008) were able to show that the weak temperature dependence is consistent with the picture of interband transitions in small gap SWNTs if there is an NT-to-NT variation of the chemical potential. Based on a simple model of an ensemble of two-level systems, they estimated a value of $100 \mathrm{meV}$ for the variation of the chemical potential. This value is compatible with the chemical potential variations found by transport and Raman measurements of NTs.

The THz conductivity of photoexcited carbon NTs has been investigated independently by several groups (Perfetti et al., 2006; Beard et al., 2008; Kampfrath et al., 2008; Xu et al., 2009). Distinct response was observed and based on these results controversial conclusions were drawn regarding the primary product of photoexcitation in SWNTs. In the study of high pressure CO grown NT films in a broad $\mathrm{THz}$ frequency range, Perfetti et al. (2006), Kampfrath et al. (2008), and coworkers observed the absence of the characteristic free-carrier response-a negative real part of the complex dielectric function-in the pump-induced optical conductivity. The absence of free-electron response was attributed by them to the strongly bound excitons in SWNTs with large energy gaps as the primary photo-excitation product (Perfetti et al., 2006). They also observed an enhanced transmission of the $\mathrm{THz}$ radiation in photoexcited SWNT films. This was explained as photo-induced bleaching of the interband transitions in the small gap NTs (Kampfrath et al., 2008). Beard et al. (2008) investigated densely packed films of both mostly semiconducting and metallic SWNTs produced by laser vaporization using a $\mathrm{THz}$ probe of 0.4 2.5 THz. In contrast, they observed a Drude-Smith-like response in the photoinduced conductivity and attributed it to free carriers. The result was further supported by a comparison to the observed dark conductivity in chemically doped NT films. Based on a detailed analysis they concluded that free carriers are generated with $>60 \%$ yields by a linear exciton dissociation process that occurs within $\sim 1$ ps after photoexcitation and is independent of the excitation wavelength or tube type.

The disagreement among these independent studies could be due to the differences in the different samples. To avoid intertube interactions, $\mathrm{Xu}$ et al. (2009) investigated isolated SWNTs with a THz probe of up to $2.5 \mathrm{THz}$. Similar to the study by Perfetti et al. (2006), a free-electron response was found absent in the photoinduced conductivity. The photoinduced complex dielectric function was described by a Lorentzian absorption term peaked around $11 \mathrm{meV}$ and a reduced conductivity that is flat within the frequency window of the THz probe. Unlike in Perfetti et al. (2006), they assigned both terms to the same mechanism (photoexcited excitons) with the two contributions corresponding to internal transitions of excitons and exciton-induced reduction in the intrinsic conductivity of the NT sample. All of the above independent studies seem to be compatible with the hypothesis that excitons are generated upon photoexcitation; efficient exciton dissociation into free charges is expected to occur at the heterojunctions of NTs of different types, and such exciton dissociation is absent in carefully separated NTs.

\section{SUMMARY AND OUTLOOK}

It is apparent from this review that time-resolved terahertz spectroscopy has developed as an extremely versatile and powerful tool in the study of charge carrier dynamics in both semiconductor bulk materials, and, perhaps more importantly, semiconductor nanostructures. It is clear that, given our current state of understanding of $\mathrm{THz}$ spectroscopy to these types of systems, future applications of time-resolved terahertz spectroscopy can focus on systems of increasing complexity, such as those encountered in photovoltaic devices where different semiconductor phases are intermixed on nanometer lengths scales (Esenturk et al., 2009). Such complex systems may include, for instance, semiconductor nanostructures (quantum dots or nanorods) embedded in an organic polymer semiconductor phase and quantum dot or dye-sensitized transition metal oxide systems (Tiwana et al., 2009; Nemec, Kuzel, and Sundstrom, 2010; Nemec, Rochford et al., 2010; Pijpers et al., 2010, 2011). The ability to monitor exciton and carrier dynamics on ultrafast time scales, across a wide frequency range, without the necessity to apply contacts to the material makes time-resolved terahertz spectroscopy a rather unique tool for studying carrier dynamics, for instance, allowing us to monitor the conversion of excitons into free charge carriers and vice versa.

An interesting aspect of $\mathrm{THz}$ radiation interacting with photoexcited semiconductors that has not been discussed in this review is the possibility to optically tune the plasma frequency in highly ordered nanostructured semiconductors, which has opened a new field of $\mathrm{THz}$ plasmonics (Rivas et al., 2004; Isaac, Rivas et al., 2008; ) and metamaterials (Chen, Padilla et al., 2007; Chen et al., 2008). The optical excitation of appropriately designed semiconductor structures 
allows for the (ultra)fast switching of $\mathrm{THz}$ transmission at specific frequencies with a high degree of control (Hendry et al., 2008; Azad et al., 2009; Berrier et al., 2010).

There are also several recent technical developments that will allow for improved experiments and completely new types of experiments. These new developments can roughly be classified into three classes: increased $\mathrm{THz}$ bandwidth (Kono et al., 2002; Sell, Scheu et al., 2008), increased $\mathrm{THz}$ intensities (peak field strengths) (Bartel et al., 2005; Hebling, Yeh, Hoffman, and Nelson, 2008), and increased stability of the sources (Adler et al., 2007). The increased intensities can be achieved by high-intensity laser sources combined with large-aperture emitters, tilted-pulse-front excitation in pyroelectrics (Hebling, Yeh, Hoffman, and Nelson, 2008), free-electron lasers ( $\mathrm{Su}$ et al., 2003), four-wave mixing in plasma (Kim et al., 2008), or difference frequency mixing of two synchronized, near-degenerate laser sources (Sell, Leitenstorfer, and Huber, 2008). The latter two approaches also inherently provide increased bandwidth, spanning to frequencies as high as $100 \mathrm{THz}\left(\sim 3000 \mathrm{~cm}^{-1}\right)$ allowing for phase-sensitive time-resolved transmission measurements approaching the near-infrared (Krauss et al., 2010), thus providing ready access to excitonic resonances, high-frequency vibrational modes and resonances associated with various types of transport, different from Drude-like.

The high intensities of improved and novel $\mathrm{THz}$ sources have already allowed for a whole range of novel experiments under strong nonequilibrium conditions, including, for example, Rabi flopping between excitonic states (Leinss et al., 2008; Steiner et al., 2008) and intersubband states in quantum wells (Luo, Reimann, Woerner, Elsaesser et al., 2004), nonlinear phase-resolved multidimensional spectroscopies in the THz range (Kuehn et al., 2009), high-field-accelerated charge carrier effects like impact ionization (Hoffmann et al., 2009b) and intervalley scattering (Su et al., 2009), and severe lattice distortions induced by high $\mathrm{THz}$ fields (Qi et al., 2009). The high intensities will also become useful to perform $\mathrm{THz}$ probe measurements in the near-field (Chan et al., 2007; Keilmann et al., 2009) using, for instance, scanning near field techniques utilizing metallic tips (Huber et al., 2008; Huber et al., 2009) or nanometer apertures (Adam et al., 2008; Seo et al., 2009). Near-field measurements promise to resolve an issue we have not addressed so far: $\mathrm{THz}$ spectroscopy is suitable to probe charge carrier dynamics in nanoscale systems. However, when used as a far-field probe of carriers, its spatial resolution is at best in the range of tens of microns owing to the diffraction limit of the probe radiation. Hence, current standard $\mathrm{THz}$ systems can merely probe ensemble properties and do not provide direct information about the local conductivity that is governed by the local charge carrier behavior. The possibility to probe conductivities on very short length and time scales will lift this limitation.

Finally, the superior stability achieved by the latest generation of fiber lasers (Adler et al., 2007) is starting to approach the level at which the quantum properties of lightmatter interactions become significant (Gunter et al., 2009). It is becoming possible to detect $\mathrm{THz}$ radiation at the single photon signal-to-noise levels (Hashiba et al., 2010), bringing $\mathrm{THz}$ quantum optics close to realization.

\section{ACKNOWLEDGMENTS}

This work is part of the research program of the "Stichting voor Fundamenteel Onderzoek der Materie (FOM)," which is financially supported by the "Nederlandse organisatie voor Wetenschappelijk Onderzoek (NWO)." J. S. acknowledges support from the National Science Foundation under Grant No. DMR-0907477 at Case Western Reserve University.

\section{REFERENCES}

Abrahams, E., P.W. Anderson, D.C. Licciardello, and T. V. Ramakrishnan, 1979, Phys. Rev. Lett. 42, 673.

Adam, A. J. L., J. M. Brok, M. A. Seo, K. J. Ahn, D. S. Kim, J. H. Kang, Q. H. Park, M. Nagel, andP.C. M. Planken, 2008, Opt. Express 16, 7407.

Adler, F., A. Sell, F. Sotier, R. Huber, and A. Leitenstorfer, 2007, Opt. Lett. 32, 3504.

Ai, X., M. C. Beard, K. P. Knutsen, S. E. Shaheen, G. Rumbles, and R. J. Ellingson, 2006, J. Phys. Chem. B 110, 25462.

Ambegaokar, V., B. I. Halperin, and J. S. Langer, 1971, Phys. Rev. B 4, 2612.

Anderson, P. W., 1958, Phys. Rev. 109, 1492.

Armstrong, M.R., E. J. Reed, K. Y. Kim, J.H. Glownia, W. M. Howard, E. L. Piner, and J. C. Roberts, 2009, Nature Phys. 5, 285.

Auston, D., 1983, Picosecond Photoconductors, Physical Properties and Applications, Picosecond Optoelectronic Devices (Academic Press, New York).

Auston, D. H., K.P. Cheung, and P. R. Smith, 1984, Appl. Phys. Lett. 45, 284.

Averitt, R. D., and A. J. Taylor, 2002, J. Phys. Condens. Matter 14, R1357.

Axt, V. M., and T. Kuhn, 2004, Rep. Prog. Phys. 67, 433.

Azad, A. K., H. T. Chen, S. R. Kasarla, A. J. Taylor, Z. Tian, X. C. Lu, W. Zhang, H. Lu, A. C. Gossard, and J. F. O'Hara, 2009, Appl. Phys. Lett. 95, 011105.

Backus, S., C. G. Durfee, M. M. Murnane, and H. C. Kapteyn, 1998, Rev. Sci. Instrum. 69, 1207.

Bartal, B., I.Z. Kozma, A. G. Stepanov, G. Almasi, J. Kuhl, E. Riedle, and J. Hebling, 2006, Appl. Phys. B 86, 419.

Bartel, T., P. Gaal, K. Reimann, M. Woerner, and T. Elsaesser, 2005, Opt. Lett. 30, 2805.

Beard, M. C., J. L. Blackburn, and M. J. Heben, 2008, Nano Lett. 8, 4238.

Beard, M. C., G. M. Turner, J. E. Murphy, O. I. Micic, M. C. Hanna, A. J. Nozik, and C. A. Schmuttenmaer, 2003, Nano Lett. 3, 1695.

Beard, M. C., G. M. Turner, and C. A. Schmuttenmaer, 2000, Phys. Rev. B 62, 15764.

Beard, M. C., G. M. Turner, and C. A. Schmuttenmaer, 2002a, Nano Lett. 2, 983.

Beard, M. C., G. M. Turner, and C.A. Schmuttenmaer, 2002b, J. Phys. Chem. B 106, 7146.

Benicewicz, P. K., J. P. Roberts, and A. J. Taylor, 1994, J. Opt. Soc. Am. B 11, 2533.

Berrier, A., R. Ulbricht, M. Bonn, and J.G. Rivas, 2010, Opt. Express 18, 23226.

Birch, J. R., C. C. Bradley, and M. F. Kimmitt, 1974, Infrared Phys. 14, 189.

Blanchard, F., et al., 2007, Opt. Express 15, 13212.

Bockrath, M., D. H. Cobden, J. Lu, A. G. Rinzler, R. E. Smalley, L. Balents, and P. L. McEuen, 1999, Nature (London) 397, 598.

Bockrath, M., D. H. Cobden, P. L. McEuen, N. G. Chopra, A. Zettl, A. Thess, and R. E. Smalley, 1997, Science 275, 1922. 
Bos, F. C., and D. M. Burland, 1987, Phys. Rev. Lett. 58, 152.

Boucaud, P., K.S. Gill, J.B. Williams, M.S. Sherwin, W. V. Schoenfeld, and P. M. Petroff, 2000, Appl. Phys. Lett. 77, 510.

Bratschitsch, R., T. Muller, G. Strasser, and K. Unterrainer, 2002, Physica E (Amsterdam) 13, 908.

Brinza, M., J. Willekens, M.L. Benkhedir, E. V. Emelianova, and G. J. Adriaenssens, 2005, J. Mater. Sci. Mater. Electron. 16, 703.

Brucherseifer, M., P. H. Bolivar, and H. Kurz, 2002, Appl. Phys. Lett. 81, 1791.

Bruggemann, A., 1935, Ann. Phys. (Leipzig) 24, 636.

Button, K., 1980, Infrared and Millimeter Waves, Vol. 1 (Sources of Radiation) (Academic Press, New York).

Cai, Y., I. Brener, J. Lopata, J. Wynn, L. Pfeiffer, J. B. Stark, Q. Wu, X. C. Zhang, and J. F. Federici, 1998, Appl. Phys. Lett. 73, 444. Cao, H., T. F. Heinz, and A. Nahata, 2002, Opt. Lett. 27, 775.

Carr, G. L., M. C. Martin, W. R. McKinney, K. Jordan, G. R. Neil, and G. P. Williams, 2002, Nature (London) 420, 153.

Castro Neto, A. H., F. Guinea, N. M. R. Peres, K. S. Novoselov, and A. K. Geim, 2009, Rev. Mod. Phys. 81, 109.

Chan, W. L., J. Deibel, and D. M. Mittleman, 2007, Rep. Prog. Phys. 70, 1325.

Chang, G. Q., C.J. Divin, C.H. Liu, S. L. Williamson, A. Galvanauskas, and T.B. Norris, 2006, Opt. Express 14, 7909.

Chemla, D. S., and J. Shah, 2001, Nature (London) 411, 549.

Chen, H. T., J. F. O'Hara, A. K. Azad, A. J. Taylor, R. D. Averitt, D. B. Shrekenhamer, and W. J. Padilla, 2008, Nat. Photon. 2, 295. Chen, H. T., W. J. Padilla, J. M. O. Zide, S. R. Bank, A. C. Gossard, A. J. Taylor, and R. D. Averitt, 2007, Opt. Lett. 32, 1620.

Chen, Y. Q., M. Yamaguchi, M.F. Wang, and X. C. Zhang, 2007, Appl. Phys. Lett. 91, 251116.

Cheville, R., 2008, Terahertz Time-Domain Spectroscopy with Photoconductive Antennas, Terahertz Spectroscopy: Principles and Applications (CRC, Boca Raton).

Chin, V. W. L., R. J. Egan, and T. L. Tansley, 1992, J. Appl. Phys. 72, 1410.

Choi, H., F. Borondics, D. A. Siegel, S. Y. Zhou, M. C. Martin, A. Lanzara, and R. A. Kaindl, 2009, Appl. Phys. Lett. 94, 172102.

Choy, T., 1999, Effective Medium Theory: Principles and Applications, International Series of Monographs on Physics (Clarendon, Oxford).

Cook, D. J., and R. M. Hochstrasser, 2000, Opt. Lett. 25, 1210.

Cooke, D. G., F. A. Hegmann, Y. I. Mazur, W. Q. Ma, X. Wang, Z. M. Wang, G. J. Salamo, M. Xiao, T.D. Mishima, and M. B. Johnson, 2004, Appl. Phys. Lett. 85, 3839.

Cooke, D. G., A. N. MacDonald, A. Hryciw, J. Wang, Q. Li, A. Meldrum, and F. A. Hegmann, 2006, Phys. Rev. B 73, 193311.

Coropceanu, V., J. Cornil, D. A. da Silva, Y. Olivier, R. Silbey, and J. L. Bredas, 2007, Chem. Rev. 107, 926.

Crooker, S. A., T. Barrick, J. A. Hollingsworth, and V. I. Klimov, 2003, Acta Phys. Pol. A 104, 113.

Cundiff, S. T., 2008, Opt. Express 16, 4639.

Cunningham, P. D., and L. M. Hayden, 2008, J. Phys. Chem. C 112, 7928.

Dakovski, G. L., S. Lan, C. Xia, and J. Shan, 2007, J. Phys. Chem. C 111, 5904.

Darrow, J. T., X.C. Zhang, and D.H. Auston, 1991, Appl. Phys. Lett. 58, 25.

Davies, J., 1997, The Physics of Low-dimensional Semiconductors: An Introduction (Cambridge University, Cambridge).

Dekorsy, T., H. Auer, H. J. Bakker, H. G. Roskos, and H. Kurz, 1996, Phys. Rev. B 53, 4005.

Devreese, J., and F. Peeters, 1984, Polarons and Excitons in Polar Semiconductors and Ionic Crystals (Springer, New York).

Dressel, M., and M. Scheffler, 2006, Ann. Phys. (Leipzig) 15, 535.
Duvillaret, L., F. Garet, and J. L. Coutaz, 1996, IEEE J. Sel. Top. Quantum Electron. 2, 739.

Dyre, J. C., and T. B. Schroder, 2000, Rev. Mod. Phys. 72, 873.

Efros, A. L., and M. Rosen, 2000, Annu. Rev. Mater. Sci. 30, 475.

Eickemeyer, F., K. Reimann, M. Woerner, T. Elsaesser, S. Barbieri, C. Sirtori, G. Strasser, T. Muller, R. Bratschitsch, and K. Unterrainer, 2002, Phys. Rev. Lett. 89, 047402.

Elsaesser, T., 2004, Appl. Phys. A 79, 1627.

Elsaesser, T., and M. Woerner, 1999, Phys. Rep. 321, 254.

Esenturk, O., R. J. Kline, D. M. Deongchamp, and E. J. Heilweil, 2008, J. Phys. Chem. C 112, 10587.

Esenturk, O., J. S. Melinger, and E. J. Heilweil, 2008, J. Appl. Phys. 103, 07A920.

Esenturk, O., J. S. Melinger, P. A. Lane, and E. J. Heilweil, 2009, J. Phys. Chem. C 113, 18842.

Faist, J., L. Ajili, G. Scalari, M. Giovannini, M. Beck, M. Rochat, H. Beere, A. G. Davies, E. H. Linfield, and D. Ritchie, 2004, Phil. Trans. R. Soc. A 362, 215.

Faist, J., F. Capasso, D. L. Sivco, C. Sirtori, A. L. Hutchinson, and A. Y. Cho, 1994, Science 264, 553.

Fattinger, C., and D. Grischkowsky, 1989, Appl. Phys. Lett. 54, 490.

Fekete, L., P. Kuzel, H. Nemec, F. Kadlec, A. Dejneka, J. Stuchlik, and A. Fejfar, 2009, Phys. Rev. B 79, 115306.

Fermann, M.E., and I. Hartl, 2009, IEEE J. Sel. Top. Quantum Electron. 15, 191.

Feynman, R., 1970, Feynman Lectures on Physics (Addison-Wesley, Reading, MA), Vol. 2, Chap. 32.

Frohlich, H., H. Pelzer, and S. Zienau, 1950, Philos. Mag., 221.

Furman, S., 1996, Basics of Optics of Multilayer Systems (World Scientific, Singapore).

Gaal, P., W. Kuehn, K. Reimann, M. Woerner, T. Elsaesser, and R. Hey, 2007, Nature (London) 450, 1210.

Galbraith, I., et al., 2005, Phys. Rev. B 71, 073302.

Gaponenko, S., 1998, Optical Properties of Semiconductor Nanocrystals (Cambridge University, Cambridge).

Garnett, J. C. M., 1904, Phil. Trans. R. Soc. A 203, 385.

Geim, A. K., 2009, Science 324, 1530.

George, P. A., J. Strait, J. Dawlaty, S. Shivaraman, M. Chandrashekhar, F. Rana, and M. G. Spencer, 2008, Nano Lett. 8, 4248 .

Gittleman, J. I., and B. Abeles, 1977, Phys. Rev. B 15, 3273.

Golde, D., M. Wagner, D. Stehr, H. Schneider, M. Helm, A. M. Andrews, T. Roch, G. Strasser, M. Kira, and S. W. Koch, 2009, Phys. Rev. Lett. 102, 127403.

Granqvist, C. G., and O. Hunderi, 1978, Phys. Rev. B 18, 2897.

Greene, B. I., J.F. Federici, D. R. Dykaar, A. F. J. Levi, and L. Pfeiffer, 1991, Opt. Lett. 16, 48.

Grischkowsky, D., 1993, Nonlinear Generation of Subpicosecond Pulses of $\mathrm{THz}$ Electromagnetic Radiation by OptoelectronicsApplications to Time-Domain Spectroscopy, Frontiers in Nonlinear Optics (Institute of Physics, Bristol/Philadelphia).

Grischkowsky, D., S. Keiding, M. Vanexter, and C. Fattinger, 1990, J. Opt. Soc. Am. B 7, 2006.

Grozema, F. C., P. T. van Duijnen, Y. A. Berlin, M. A. Ratner, and L. D. A. Siebbeles, 2002, J. Phys. Chem. B 106, 7791.

Gunter, G., et al., 2009, Nature (London) 458, 178.

Han, P. Y., and X. C. Zhang, 2001, Meas. Sci. Technol. 12, 1747.

Harrison, P., 2002, Quantum Wells, Wires and Dots: Theoretical and Computational Physics of Semiconductor Nanostructures (John Wiley and Sons, New York).

Hashiba, H., V. Antonov, L. Kulik, A. Tzalenchuk, and S. Komiyama, 2010, Nanotechnology 21, 165203.

Hasselbeck, M. P., D. Stalnaker, L. A. Schlie, T. J. Rotter, A. Stintz, and M. Sheik-Bahae, 2002, Phys. Rev. B 65, 233203. 
Haug, H., 1994, Quantum Theory of the Optical and Electronic Properties of Semiconductors (World Scientific, Singapore).

Hebling, J., K. L. Yeh, M. C. Hoffmann, B. Bartal, and K. A. Nelson, 2008, J. Opt. Soc. Am. B 25, B6.

Hebling, J., K. L. Yeh, M. C. Hoffmann, and K. A. Nelson, 2008, IEEE J. Sel. Top. Quantum Electron. 14, 345.

Hegmann, F. A., R. R. Tykwinski, K.P.H. Lui, J.E. Bullock, and J. E. Anthony, 2002, Phys. Rev. Lett. 89, 227403.

Hendry, E., F. J. Garcia-Vidal, L. Martin-Moreno, J. G. Rivas, M. Bonn, A. P. Hibbins, and M. J. Lockyear, 2008, Phys. Rev. Lett. 100, 123901.

Hendry, E., M. Koeberg, and M. Bonn, 2007, Phys. Rev. B 76, 045214.

Hendry, E., M. Koeberg, B. O'Regan, and M. Bonn, 2006, Nano Lett. 6, 755.

Hendry, E., M. Koeberg, J. Pijpers, and M. Bonn, 2007, Phys. Rev. B 75, 233202.

Hendry, E., M. Koeberg, J.M. Schins, H.K. Nienhuys, V. Sundstrom, L. D. A. Siebbeles, and A. Bonn, 2005, Phys. Rev. B 71, 125201.

Hendry, E., M. Koeberg, J. M. Schins,L. D. A. Siebbeles, and M. Bonn, 2006, Chem. Phys. Lett. 432, 441.

Hendry, E., M. Koeberg, F. Wang, H. Zhang, C. D. Donega, D. Vanmaekelbergh, and M. Bonn, 2006, Phys. Rev. Lett. 96, 057408

Hendry, E., J. M. Schins, L. P. Candeias,L. D. A. Siebbeles, and M. Bonn, 2004, Phys. Rev. Lett. 92, 196601.

Hendry, E., F. Wang, J. Shan, T. F. Heinz, and M. Bonn, 2004, Phys. Rev. B 69, 081101.

Heyman, J. N., R. Kersting, and K. Unterrainer, 1998, Appl. Phys. Lett. 72, 644.

Hickey, B., G. Morgan, and M. Howson, 2001, Basic Electron Transport (Springer, New York).

Hoffmann, M. C., J. Hebling, H. Y. Hwang, K. L. Yeh, and K. A. Nelson, 2009a, Phys. Rev. B 79, 161201.

Hoffmann, M. C., J. Hebling, H. Y. Hwang, K. L. Yeh, and K. A. Nelson, 2009b, J. Opt. Soc. Am. B 26, A29.

Houard, A., Y. Liu, B. Prade, and A. Mysyrowicz, 2008, Opt. Lett. 33, 1195.

Hu, B. B., X. C. Zhang, D. H. Auston, and P. R. Smith, 1990, Appl. Phys. Lett. 56, 506.

Huber, A. J., F. Keilmann, J. Wittborn, J. Aizpurua, and R. Hillenbrand, 2008, Nano Lett. 8, 3766.

Huber, A. J., A. Ziegler, T. Kock, and R. Hillenbrand, 2009, Nature Nanotech. 4, 153.

Huber, R., A. Brodschelm, F. Tauser, and A. Leitenstorfer, 2000, Appl. Phys. Lett. 76, 3191.

Huber, R., R. A. Kaindl, B. A. Schmid, and D. S. Chemla, 2005, Phys. Rev. B 72, 161314.

Huber, R., C. Kubler, S. Tubel, A. Leitenstorfer, Q. T. Vu, H. Haug, F. Kohler, and M. C. Amann, 2005, Phys. Rev. Lett. 94, 027401.

Huber, R., B. A. Schmid, Y.R. Shen, D. S. Chemla, and R. A. Kaindl, 2006, Phys. Rev. Lett. 96, 017402.

Huber, R., F. Tauser, A. Brodschelm, M. Bichler, G. Abstreiter, and A. Leitenstorfer, 2001, Nature (London) 414, 286.

Isaac, T. H., W. L. Barnes, and E. Hendry, 2008, Appl. Phys. Lett. 93, 241115.

Isaac, T. H., J. G. Rivas, J. R. Sambles, W. L. Barnes, and E. Hendry, 2008, Phys. Rev. B 77, 113411.

Ishii, H., et al., 2003, Nature (London) 426, 540.

Jeffries, C. D., 1975, Science 189, 955.

Jeon, T. I., and D. Grischkowsky, 1997, Phys. Rev. Lett. 78, 1106.
Jeon, T. I., and D. Grischkowsky, 1998, Appl. Phys. Lett. 72, 2259.

Jeon, T. I., K. J. Kim, C. Kang, S. J. Oh, J.H. Son, K. H. An, D. J. Bae, and Y.H. Lee, 2002, Appl. Phys. Lett. 80, 3403.

Jeon, T. I., K. J. Kim, C. Kang, S. J. Oh, J. H. Son, K. H. An, D. J. Bae, and Y. H. Lee, 2003, Synth. Met. 135, 429.

Jepsen, P. U., W. Schairer, I. H. Libon, U. Lemmer, N. E. Hecker, M. Birkholz, K. Lips, and M. Schall, 2001, Appl. Phys. Lett. 79, 1291.

Jiang, Z., F. G. Sun, Q. Chen, and X. C. Zhang, 1999, Appl. Phys. Lett. 74, 1191.

Jiang, Z.P., M. Li, and X.C. Zhang, 2000, Appl. Phys. Lett. 76, 3221.

Jiang, Z. P., and X. C. Zhang, 1998, Appl. Phys. Lett. 72, 1945.

Johnson, C. J., G. H. Sherman, and R. Weil, 1969, Appl. Opt. 8, 1667.

Jorio, A., G. Dresselhaus, and M. Dresselhaus, 2008, Carbon Nanotubes: Advanced Topics in the Synthesis, Structure, Properties and Applications (Springer, New York).

Jukam, N., et al., 2009, Appl. Phys. Lett. 94, 251108.

Jukam, N., et al., 2008, Appl. Phys. Lett. 93, 101115.

Kadoya, Y., and K. Hirakawa, 2005, in Terahertz Radiation from Bulk and Quantum Semiconductor Structures, Terahertz Optoelectronics (Springer, Berlin), Vol. 97, pp. 117-156.

Kaindl, R. A., F. Eickemeyer, M. Woerner, and T. Elsaesser, 1999, Appl. Phys. Lett. 75, 1060.

Kaindl, R. A., D. Hagele, M. A. Carnahan, and D. S. Chemla, 2009, Phys. Rev. B 79, 045320.

Kaindl, R. A., D. Hagele, M. A. Carnahan, R. Lovenich, and D. S. Chemla, 2003, Physica B (Amsterdam) 238, 451.

Kaindl, R. A., R. Huber, B. A. Schmid, M. A. Carnahan, D. Hagele, and D. S. Chemla, 2006, Physica B (Amsterdam) 243, 2414.

Kampfrath, T., L. Perfetti, F. Schapper, C. Frischkorn, and M. Wolf, 2005, Phys. Rev. Lett. 95, 187403.

Kampfrath, T., K. von Volkmann, C. M. Aguirre, P. Desjardins, R. Martel, M. Krenz, C. Frischkorn, M. Wolf, and L. Perfetti, 2008, Phys. Rev. Lett. 101, 267403.

Karpowicz, N., X. F. Lu, and X.C. Zhang, 2009, J. Mod. Opt. 56, 1137.

Katayama, I., R. Akai, M. Bito, H. Shimosato, K. Miyamoto, H. Ito, and M. Ashida, 2010, Appl. Phys. Lett. 97, 021105.

Katzenellenbogen, N., and D. Grischkowsky, 1992, Appl. Phys. Lett. 61, 840.

Kawada, Y., T. Yasuda, H. Takahashi, and S. I. Aoshima, 2008, Opt. Lett. 33, 180.

Keilmann, F., A.J. Huber, and R. Hillenbrand, 2009, J. Infrared Millimeter Waves 30, 1255.

Kersting, R., R. Bratschitsch, G. Strasser, K. Unterrainer, and J. N. Heyman, 2000, Opt. Lett. 25, 272.

Kim, D. S., and D. S. Citrin, 2006, Appl. Phys. Lett. 88, 161117.

Kim, K. Y., A. J. Taylor, J.H. Glownia, and G. Rodriguez, 2008, Nat. Photon. 2, 605.

Kim, K. Y., B. Yellampalle, A. J. Taylor, G. Rodriguez, and J. H. Glownia, 2007, Opt. Lett. 32, 1968.

Kindt, J. T., and C. A. Schmuttenmaer, 1999, J. Chem. Phys. 110, 8589.

Kira, M., W. Hoyer, and S.W. Koch, 2004, Solid State Commun. 129, 733 .

Kira, M., W. Hoyer, T. Stroucken, and S. W. Koch, 2001, Phys. Rev. Lett. 87, 176401.

Kira, M., F. Jahnke, and S. W. Koch, 1998, Phys. Rev. Lett. 81, 3263.

Kira, M., and S. W. Koch, 2004, Phys. Rev. Lett. 93, 076402.

Kira, M., and S. W. Koch, 2006, Prog. Quantum Electron. 30, 155. 
Kittel, C., 1995, Introduction to Solid State Physics (Wiley, New York), p. 276.

Klimov, V. I., 2007, Annu. Rev. Phys. Chem. 58, 635.

Klingshirn, C., 2006, Semiconductor Optics (Springer, New York).

Knoesel, E., M. Bonn, J. Shan, F. Wang, and T. F. Heinz, 2004, J. Chem. Phys. 121, 394.

Koch, S. W., M. Kira, G. Khitrova, and H. M. Gibbs, 2006, Nature Mater. 5, 523.

Koeberg, M., E. Hendry, J. M. Schins, H. A. van Laarhoven, C. F. J. Flipse, K. Reimann, M. Woerner, T. Elsaesser, and M. Bonn, 2007, Phys. Rev. B 75, 195216.

Kohler, R., A. Tredicucci, F. Beltram, H.E. Beere, E. H. Linfield, A. G. Davies, D. A. Ritchie, R. C. Iotti, and F. Rossi, 2002, Nature (London) 417, 156.

Konenkamp, R., G. Priebe, and B. Pietzak, 1999, Phys. Rev. B 60, 11804.

Kono, S., M. Tani, and K. Sakai, 2002, IEE Proc. - Optoelectron. 149, 105.

Krauss, G., S. Lohss, T. Hanke, A. Sell, S. Eggert, R. Huber, and A. Leitenstorfer, 2009, Nat. Photon. 4, 33.

Kretschmann, E., 1972, Opt. Commun. 5, 331.

Kroll, J., J. Darmo, K. Unterrainer, S.S. Dhillon, C. Sirtori, X. Marcadet, and M. Calligaro, 2007, Appl. Phys. Lett. 91, 161108

Kroll, J., S. S. Dhillon, X. Marcadet, M. Calligaro, C. Sirtori, and K. Unterrainer, 2007, Nature (London) 449, 698.

Krotkus, A., and J.L. Coutaz, 2005, Semicond. Sci. Technol. 20, S142.

Kruger, A., 2008, Carbon Materials and Nanotechnolgy (Wiley$\mathrm{VCH}$, New York).

Kubo, R., 1957, J. Phys. Soc. Jpn. 12, 570.

Kubouchi, M., K. Yoshioka, R. Shimano, A. Mysyrowicz, and M. Kuwata-Gonokami, 2005, Phys. Rev. Lett. 94, 016403.

Kuehn, W., et al., 2008, Appl. Phys. Lett. 93, 151106.

Kuehn, W., K. Reimann, M. Woerner, and T. Elsaesser, 2009, J. Chem. Phys. 130.

Laarhoven, H. A. V.,C. F. J. Flipse, M. Koeberg, M. Bonn, E. Hendry, G. Orlandi, O.D. Jurchescu, T. T. M. Palstra, and A. Troisi, 2008, J. Chem. Phys. 129, 044704.

Lee, C. H., G. Yu, D. Moses, and A. J. Heeger, 1994, Phys. Rev. B 49, 2396.

Lee, Y., 2009, Principles of Terahertz Science and Technology (Lecture Notes in Physics) (Springer, New York).

Leinss, S., T. Kampfrath, K. von Volkmann, M. Wolf, J. T. Steiner, M. Kira, S. W. Koch, A. Leitenstorfer, and R. Huber, 2008, Phys. Rev. Lett. 101, 246401.

Leitenstorfer, A., S. Hunsche, J. Shah, M. C. Nuss, and W. H. Knox, 1999, Phys. Rev. Lett. 82, 5140.

Leitenstorfer, A., S. Hunsche, J. Shah, M. C. Nuss, and W. H. Knox, 2000, Phys. Rev. B 61, 16642.

Liu, P. Q., A. J. Hoffman, M. D. Escarra, K. J. Franz, J. B. Khurgin, Y. Dikmelik, X. J. Wang, J. Y. Fan, and C. F. Gmachl, 2010, Nat. Photon. 4, 95.

Lloyd-Hughes, J., Y. L. Delley, G. Scalari, M. Fischer, V. Liverini, M. Beck, and J. Faist, 2009, J. Appl. Phys. 106, 093104.

Lloyd-Hughes, J., S. K. E. Merchant, L. Sirbu, I. M. Tiginyanu, and M. B. Johnston, 2008, Phys. Rev. B 78.

Loffler, T., T. Hahn, M. Thomson, F. Jacob, and H. G. Roskos, 2005, Opt. Express 13, 5353.

Lovenich, R., C. W. Lai, D. Hagele, D. S. Chemla, and W. Schafer, 2002, Phys. Rev. B 66, 045306.

Lu, X. F., N. Karpowicz, Y. Q. Chen, and X. C. Zhang, 2008, Appl. Phys. Lett. 93, 261106.
Luo, C., K. Reimann, M. Woerner, and T. Elsaesser, 2004, Appl. Phys. A 78, 435.

Luo, C. W., K. Reimann, M. Woerner, T. Elsaesser, R. Hey, and K. H. Ploog, 2004, Phys. Rev. Lett. 92, 047402.

Mak, K. F., M. Y. Sfeir, Y. Wu, C. H. Lui, J. A. Misewich, and T. F. Heinz, 2008, Phys. Rev. Lett. 101, 196405.

Mandal, P. K., and V. Chikan, 2007, Nano Lett. 7, 2521.

Mayou, D., 2000, Phys. Rev. Lett. 85, 1290.

McIntosh, K. A., E. R. Brown, K. B. Nichols, O. B. McMahon, W. F. DiNatale, and T. M. Lyszczarz, 1995, Appl. Phys. Lett. 67, 3844.

Merchant, S. K. E., J. Lloyd-Hughes, L. Sirbu, I. M. Tiginyanu, P. Parkinson, L. M. Herz, and M. B. Johnston, 2008, Nanotechnology 19, 395704.

Messner, C., H. Kostner, R. A. Hopfel, and K. Unterrainer, 2001, J. Opt. Soc. Am. B 18, 1369.

Mickan, S. P., K. S. Lee, T. M. Lu, J. Munch, D. Abbott, and X. C. Zhang, 2002, Microelectron. J. 33, 1033.

Mikulics, M., E. A. Michael, M. Marso, M. Lepsa, A. van der Hart, H. Luth, A. Dewald, S. Stancek, M. Mozolik, and P. Kordos, 2006, Appl. Phys. Lett. 89, 071103.

Moses, D., D. Comoretto, C. H. Lee, and A. J. Heeger, 1997, Synth. Met. 84, 559.

Mott, N. F., 1968, J. Non-Cryst. Solids 1, 1.

Mott, N. F., 1969, Philos. Mag. 19, 835.

Muller, T., W. Parz, K. Unterrainer, S. Sauvage, J. Houel, P. Boucaud, A. Miard, and A. Lemaitre, 2008, Phys. Rev. B 77, 035314.

Murdin, B. N., 2009, Contemp. Phys. 50, 391.

Murphy, J. E., M. C. Beard, and A. J. Nozik, 2006, J. Phys. Chem. B 110, 25455.

Murray, W. A., and W. L. Barnes, 2007, Adv. Mater. 19, 3771.

Nahata, A., D. H. Auston, T.F. Heinz, and C. J. Wu, 1996, Appl. Phys. Lett. 68, 150.

Nahata, A., A. S. Weling, and T. F. Heinz, 1996, Appl. Phys. Lett. 69, 2321.

Nemec, H., F. Kadlec, C. Kadlec, P. Kuzel, and P. Jungwirth, 2005, J. Chem. Phys. 122, 104503.

Nemec, H., F. Kadlec, and P. Kuzel, 2002, J. Chem. Phys. 117, 8454.

Nemec, H., F. Kadlec, S. Surendran, P. Kuzel, and P. Jungwirth, 2005, J. Chem. Phys. 122.

Nemec, H., P. Kuzel, and V. Sundstrom, 2009, Phys. Rev. B 79.

Nemec, H., P. Kuzel, and V. Sundstrom, 2010, J. Photochem. Photobiol. A, Chem. 215, 123.

Nemec, H., H. K. Nienhuys, E. Perzon, F. L. Zhang, O. Inganas, P. Kuzel, and V. Sundstrom, 2009, Phys. Rev. B 79.

Nemec, H., H. K. Nienhuys, F. Zhang, O. Inganas, A. Yartsev, and V. Sundstrom, 2008, J. Phys. Chem. C 112, 6558.

Nemec, H., J. Rochford, O. Taratula, E. Galoppini, P. Kuzel, T. Polivka, A. Yartsev, and V. Sundstrom, 2010, Phys. Rev. Lett. 104, 197401.

Nienhuys, H. K., and V. Sundstrom, 2005a, Appl. Phys. Lett. 87, 012101.

Nienhuys, H. K., and V. Sundstrom, 2005b, Phys. Rev. B 71, 235110.

Novoselov, K. S., A. K. Geim, S. V. Morozov, D. Jiang, Y. Zhang, S. V. Dubonos, I. V. Grigorieva, and A. A. Firsov, 2004, Science 306, 666 .

Nygard, J., D. H. Cobden, and P. E. Lindelof, 2000, Nature (London) 408, 342.

Odom, T. W., J.L. Huang, C. L. Cheung, and C. M. Lieber, 2000, Science 290, 1549.

O'Hara, K. E., and J.P. Wolfe, 2000, Phys. Rev. B 62, 12909. 
Oregan, B., and M. Gratzel, 1991, Nature (London) 353, 737.

Orenstein, J., and M. A. Kastner, 1981, Solid State Commun. 40, 85.

Ostroverkhova, O., D. G. Cooke, S. Shcherbyna, R. F. Egerton, F. A.

Hegmann, R. R. Tykwinski, and J. E. Anthony, 2005, Phys. Rev. B 71, 035204 .

Othonos, A., 1998, J. Appl. Phys. 83, 1789.

Pacebutas, V., A. Biciunas, S. Balakauskas, A. Krotkus, G. Andriukaitis, D. Lorenc, A. Pugzlys, and A. Baltuska, 2010, Appl. Phys. Lett. 97, 031111.

Parkinson, P., H. J. Joyce, Q. Gao, H. H. Tan, X. Zhang, J. Zou, C. Jagadish, L. M. Herz, and M. B. Johnston, 2009, Nano Lett. 9, 3349.

Parkinson, P., J. Lloyd-Hughes, Q. Gao, H. H. Tan, C. Jagadish, M. B. Johnston, and L. M. Herz, 2007, Nano Lett. 7, 2162.

Parkinson, P., J. Lloyd-Hughes, M. B. Johnston, and L. M. Herz, 2008, Phys. Rev. B 78, 115321.

Perfetti, L., T. Kampfrath, F. Schapper, A. Hagen, T. Hertel, C. M. Aguirre, P. Desjardins, R. Martel, C. Frischkorn, and M. Wolf, 2006, Phys. Rev. Lett. 96, 027401.

Pierson, H., 1993, Handbook of Carbon, Graphite, Diamond, and Fullerenes: Properties, Processing, and Applications (Noyes Publications, Park Ridge, N.J.).

Pijpers, J. J. H., et al., 2007, J. Phys. Chem. C 111, 4146.

Pijpers, J. J. H., et al., 2008, J. Phys. Chem. C 112, 4783.

Pijpers, J. J. H., R. Koole, W. H. Evers, A. J. Houtepen, S. Boehme, C. D. Donega, D. Vanmaekelbergh, and M. Bonn, 2010, J. Phys. Chem. C, 114, 18866

Pijpers, J. J. H., R. Ulbricht, S. Derossi,J. N. H. Reek, and M. Bonn, 2011, J. Phys. Chem. C 115, 2578.

Pijpers, J. J. H., R. Ulbricht, K. J. Tielrooij, A. Osherov, Y. Golan, C. Delerue, G. Allan, and M. Bonn, 2009, Nature Phys. 5, 811.

Prasankumar, R. P., P. C. Upadhya, and A.J. Taylor, 2009, Phys. Status Solidi B 246, 1973.

Prins, P., F.C. Grozema, J.M. Schins, S. Patil, U. Scherf, and L. D. A. Siebbeles, 2006, Phys. Rev. Lett. 96, 146601.

Qi, T. T., Y.H. Shin, K. L. Yeh, K. A. Nelson, and A. M. Rappe, 2009, Phys. Rev. Lett. 102, 247603.

Rana, F., P. A. George, J. H. Strait, J. Dawlaty, S. Shivaraman, M. Chandrashekhar, and M.G. Spencer, 2009, Phys. Rev. B 79, 115447.

Razzari, L., et al., 2009, Phys. Rev. B 79, 193204.

Reich, S., C. Thomsen, and J. Maultzsch, 2004, Carbon Nanotubes: Basic Concepts and Physical Properties (Wiley-VCH, New York).

Reimann, K., 2007, Rep. Prog. Phys. 70, 1597.

Riikonen, S., I. Romero, and F. J. Garcia de Abajo, 2005, Phys. Rev. B 71, 235104.

Ritchie, R. H., 1957, Phys. Rev. 106, 874.

Rivas, J. G., M. Kuttge, P. H. Bolivar, H. Kurz, and J. A. SanchezGil, 2004, Phys. Rev. Lett. 93, 256804.

Sakai, K., 2005, Terahertz Optoelectronics (Springer, Berlin).

Scalari, G., C. Walther, M. Fischer, R. Terazzi, H. Beere, D. Ritchie, and J. Faist, 2009, Laser and Photonics Reviews 3, 45.

Scher, H., and E. W. Montroll, 1975, Phys. Rev. B 12, 2455.

Schins, J. M., E. Hendry, M. Bonn, and H. G. Muller, 2007, J. Chem. Phys. 97.

Schins, J. M., 2010, Appl. Phys. Lett. 97, 172110.

Schmuttenmaer, C. A., 2004, Chem. Rev. 104, 1759.

Sell, A., A. Leitenstorfer, and R. Huber, 2008, Opt. Lett. 33, 2767.

Sell, A., R. Scheu, A. Leitenstorfer, and R. Huber, 2008, Appl. Phys. Lett. 93, 251107.

Seo, M. A., et al., 2009, Nat. Photon. 3, 152.

Shah, J., 1999, Ultrafast Spectroscopy of Semiconductors and Semiconductor Nanostructures (Springer, New York).
Shah, J., M. Combescot, and A. H. Dayem, 1977, Phys. Rev. Lett. 38, 1497.

Shan, J., and T.F. Heinz, 2004, Terahertz Radiation From Semiconductors, Topics in Applied Physics Vol. 92 (Springer, Berlin/Heidelberg), pp. 1-56.

Shan, J., F. Wang, E. Knoesel, M. Bonn, and T. F. Heinz, 2003, Phys. Rev. Lett. 90.

Shan, J., C. Weiss, R. Wallenstein, R. Beigang, and T. F. Heinz, 2001, Opt. Lett. 26, 849.

Shan, J., A. S. Weling, E. Knoesel, L. Bartels, M. Bonn, A. Nahata, G. A. Reider, and T. F. Heinz, 2000, Opt. Lett. 25, 426.

Shen, Y. C., P. C. Upadhya, H. E. Beere, E. H. Linfield, A. G. Davies, I. S. Gregory, C. Baker, W. R. Tribe, and M. J. Evans, 2004, Appl. Phys. Lett. 85, 164.

Shik, A., 1999, Quantum Wells: Physics and Electronics of TwoDimensional Systems (World Scientific, Singapore).

Smith, A., and D. Dutton, 1958, J. Opt. Soc. Am. 48, 1007.

Smith, N. V., 2001, Phys. Rev. B 64, 155106.

Smith, P. R., D. H. Auston, and M. C. Nuss, 1988, IEEE J. Quantum Electron. 24, 255.

Spanier, J. E., and I. P. Herman, 2000, Phys. Rev. B 61, 10437.

Steiner, J. T., M. Kira, and S. W. Koch, 2008, Phys. Rev. B 77.

Stepanov, A. G., L. Bonacina, S. V. Chekalin, and J. P. Wolf, 2008, Opt. Lett. 33, 2497.

Strait, J.H., P. A. George, M. Levendorf, M. Blood-Forsythe, F. Rana, and J. Park, 2009, Nano Lett. 9, 2967.

Stroud, D., 1975, Phys. Rev. B 12, 3368.

Su, F. H., et al., 2009, Opt. Express 17, 9620.

Su, M. Y., S. G. Carter, M. S. Sherwin, A. Huntington, and L. A. Coldren, 2003, Phys. Rev. B 67, 125307.

Suzuki, T., and R. Shimano, 2009, Phys. Rev. Lett. 103, 057401.

Tans, S. J., M. H. Devoret, H. J. Dai, A. Thess, R. E. Smalley, L. J. Geerligs, and C. Dekker, 1997, Nature (London) 386, 474.

Thomson, M.D., M. Kress, T. Loffler, and H. G. Roskos, 2007, Laser and Photonics Reviews 1, 349.

Thorsmolle, V. K., R. D. Averitt, X. Chi, D. J. Hilton, D. L. Smith, A. P. Ramirez, and A. J. Taylor, 2004, Appl. Phys. Lett. 84, 891.

Tiwana, P., P. Parkinson, M. B. Johnston, H. J. Snaith, and L. M. Herz, 2009, J. Phys. Chem. C 114, 1365.

Troisi, A., and G. Orlandi, 2006, Phys. Rev. Lett. 96, 086601.

Turner, G. M., M. C. Beard, and C. A. Schmuttenmaer, 2002, J. Phys. Chem. B 106, 11716.

Vanexter, M., and D. Grischkowsky, 1990, Phys. Rev. B 41, 12140.

Vengurlekar, A. S., and S. S. Jha, 1991, Phys. Rev. B 43, 12454.

Wallace, P. R., 1947, Phys. Rev. 71, 622.

Wang, F., G. Dukovic, L.E. Brus, and T.F. Heinz, 2005, Science 308, 838 .

Wang, F., J. Shan, M. A. Islam, I. P. Herman, M. Bonn, and T.F. Heinz, 2006, Nature Mater. 5, 861.

Wang, X. F., D. J. Hilton, L. Ren, D. M. Mittleman, J. Kono, and J. L. Reno, 2007, Opt. Lett. 32, 1845.

Williams, B.S., 2007, Nat. Photon. 1, 517.

Williams, G. P., 2004, Phil. Trans. R. Soc. A 362, 403.

Williams, G. P., 2006, Rep. Prog. Phys. 69, 301.

Woggon, U., 1997, Optical Properties of Semiconductor Quantum Dots (Springer, New York).

Woolard, D., W. Loerop, and M. Shur, 2003, Terahertz Sensing Technology: Vol. 1 (Electronic Devices and Advanced Systems Technology) (World Scientific, New Jersey).

Wu, Q., and X. C. Zhang, 1995, Appl. Phys. Lett. 67, 3523.

Wu, Q., and X.C. Zhang, 1997, Appl. Phys. Lett. 70, 1784.

Wynne, K., and J. J. Carey, 2005, Opt. Commun. 256, 400. 
Xie, X., J.M. Dai, and X.C. Zhang, 2006, Phys. Rev. Lett. 96, 075005.

Xu, X. L., K. Chuang, R. J. Nicholas, M. B. Johnston, and L. M. Herz, 2009, J. Phys. Chem. C 113, 18106.

Yan, H. G., D. H. Song, K. F. Mak, I. Chatzakis, J. Maultzsch, and T. F. Heinz, 2009, Phys. Rev. B 80, 121403.

Yellampalle, B., K. Y. Kim, G. Rodriguez, J.H. Glownia, and A. J. Taylor, 2005, Appl. Phys. Lett. 87, 211109.
You, D., R. R. Jones, P. H. Bucksbaum, and D. R. Dykaar, 1993, Opt. Lett. 18, 290.

Zheng, X. M., C. V. McLaughlin, P. Cunningham, and L.M. Hayden, 2007, J. Nanoelectron. Optoelectron. 2, 58.

Zhong, Z. H., N. M. Gabor, J.E. Sharping, A.L. Gaeta, and P.L. McEuen, 2008, Nature Nanotech. 3, 201.

Zhou, Q. L., Y.L. Shi, B. Jin, and C.L. Zhang, 2008, Appl. Phys. Lett. 93. 